Travaux et documents du CIRAC

\title{
Modernisation des services publics et management social en France et en Allemagne
}

Sous la direction de

Leo KIßLER, René LASSERRE et Marie-Hélène PAUTRAT

\section{CIRAC}




\section{Modernisation des services publics et management social en France et en Allemagne}

Leo Kißler, René Lasserre et Marie-Hélène Pautrat (dir.)

DOI : 10.4000/books.cirac. 644

Éditeur : IFAEE

Année d'édition : 2007

Date de mise en ligne : 13 décembre 2017

Collection : Travaux et documents du CIRAC

ISBN électronique : 9782905518583

\section{Sbooks}

http://books.openedition.org

Édition imprimée

ISBN : 9782905518347

Nombre de pages : 142

Référence électronique

KISSLER, Leo (dir.) ; LASSERRE, René (dir.) ; et PAUTRAT, Marie-Hélène (dir.). Modernisation des services publics et management social en France et en Allemagne. Nouvelle édition [en ligne]. Cergy-Pontoise :

IFAEE, 2007 (généré le 02 octobre 2020). Disponible sur Internet : <http://books.openedition.org/cirac/ 644>. ISBN : 9782905518583. DOI : https://doi.org/10.4000/books.cirac.644.

\section{(C) IFAEE, 2007}

Conditions d'utilisation

http://www.openedition.org/6540 
Travaux et documents du CIRAC

\title{
Modernisation des services publics et management social en France et en Allemagne
}

\author{
Sous la direction de \\ Leo KIßLER, René LASSERRE et Marie-Hélène PAUTRAT
}






\section{Modernisation des services publics et management social en France et en Allemagne}

Sous la direction de

Leo KIßLER, René LASSERRE et Marie-Hélène PAUTRAT 


\section{Travaux et documents du CIRAC}

Collection dirigée par René Lasserre 


\title{
MODERNISATION DES SERVICES PUBLICS ET MANAGEMENT SOCIAL en France et en Allemagne
}

\author{
TABLE DES MATIÈRES
}

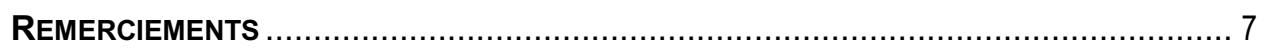

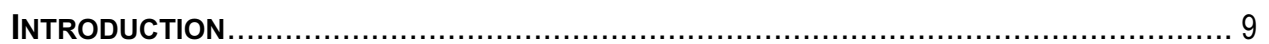

Leo Kißler, René Lasserre, Marie-Hélène Pautrat

Modernisation de l'Etat et de l'administration en France et en Allemagne :

approches, modalités et problématiques

\section{I - DÉRÉGULATION ET MODERNISATION DE L'ADMINISTRATION EN France et EN ALLEMAGne}

\section{Karsten Schneider}

La régulation des services publics en Europe et ses effets

sur la qualité des prestations, l'emploi et les conditions de travail.

\section{Pierre Bauby}

Dérégulations et privatisations dans les services publics en France :

l'exemple du secteur de l'électricité

Rina Bohle Zeller

Le secteur de l'eau en Allemagne - entre libéralisation et modernisation

\section{II - EMPLOI PUBLIC ET GESTION DES RESSOURCES HUMAINES}

\section{Sabine Kuhlmann}

La fonction publique en Allemagne :

ouverte à la réforme ou réfractaire au changement?

\section{Catherine Zaïdman}

Emploi public en France : état des lieux et perspectives d'évolution

\section{Suzanne König}

La gestion des ressources humaines dans le processus de réforme

des administrations publiques : l'exemple de la Basse-Saxe ...... 


\section{III - RELATIONS SOCIALES ET REPRÉSENTATION DU PERSONNEL}

\section{Philippe Moncourrier}

Evolution des relations sociales dans les services d'intérêt général :

l'exemple de la RATP.

\section{Hermann Hibbeler}

La représentation du personnel, acteur de la modernisation?

Expériences de pilotage partagé de la réforme

\section{Marie-Claude Kervella}

Le dialogue social dans la fonction publique d'Etat en France 


\section{Remerciements}

Cette publication est l'aboutissement d'un projet de formation-recherche intitulé "Nouveaux modes de régulation sociale dans le secteur public et les services d'intérêt général en Allemagne et en France" qui a été développé par le Centre d'Information et de Recherche sur l'Allemagne Contemporaine (CIRAC) entre 2004 et 2006, en coopération avec l'Institut de sociologie de l'Université de Marburg, principalement à l'attention des étudiants du Master Etudes européennes et de l'Ecole doctorale Droit et Sciences humaines de l'Université de Cergy-Pontoise. Le principal objectif de ce programme était d'analyser les modalités de la modernisation de l'administration et de la dérégulation des services d'intérêt général en France et en Allemagne et de mettre en évidence leurs effets sur les conditions d'emploi et de travail ainsi que sur les relations sociales dans le secteur public de part et d'autre du Rhin.

Cet ouvrage rassemble essentiellement des articles rédigés à partir des contributions que des chercheurs spécialistes et des responsables professionnels français et allemands des secteurs concernés ont présentées lors de deux journées d'études consacrées à cette problématique.

Le projet a été mené à bien grâce au concours financier qui lui a été accordé à la fois par le Centre Interdisciplinaire d'Etudes et de Recherches sur l'Allemagne (CIERA) dans le cadre de ses Programmes de formation-recherche et par la Fondation Hans-Böckler (Düsseldorf). Outre les auteurs et les traducteurs, nous tenons également à remercier Werner Zettelmeier, chargé de recherches au CIRAC, pour la contribution précieuse qu'il a apportée à la mise en forme des contributions allemandes pour l'édition française de cet ouvrage.

René Lasserre

Directeur du CIRAC 



\title{
Modernisation de l'Etat et de l'administration en France et en Allemagne : approches, modalités et problématiques
}

\author{
Leo KIßLER, René LASSERRE, Marie-Hélène PAUTRAT
}

La compétitivité économique d'un pays repose également, et sans doute de façon aujourd'hui très explicite, sur l'organisation et l'efficience du secteur public, pris ici au sens large, c'est à dire autant dans la dimension régalienne de l'Etat - les services administratifs de l'Etat - que dans son activité économique - les services publics à caractère industriel et commercial. Le secteur public doit être en mesure de générer un environnement favorable au développement de l'activité économique du pays et au maintien d'un certain niveau de cohésion sociale: qualité des infrastructures, qu'il s'agisse des voies de communication, des transports, des télécommunications, de la régularité de l'approvisionnement énergétique, etc., mais aussi, plus indirectement, du niveau et de l'adéquation de la formation de la main d'oeuvre aux besoins des entreprises et de la société, ou encore de la stabilité du contexte politico-social.

C'est plus que jamais le cas dans le contexte actuel de compétition internationale croissante liée à la globalisation des marchés dans lequel les entreprises sont conduites à recentrer au maximum leurs activités, à optimiser leur organisation logistique et leur structure de production, à externaliser des tâches et fonctions insuffisamment créatrices de valeur. Elles ne peuvent cependant développer ces stratégies de rentabilité avec de réelles perspectives de succès que dans la mesure où la qualité de leur environnement territorial est garantie. En conséquence, les entreprises sont donc aujourd'hui particulièrement tributaires de la fiabilité et de la continuité des prestations proposées dans le cadre national, régional ou local dans lequel elles sont implantées.

Cette question joue d'ailleurs un rôle de plus en plus important dans le débat actuel sur la localisation des activités industrielles et constitue un enjeu majeur des politiques économiques dont l'une des priorités essentielles consiste désormais à garantir l'attractivité des sites de production en créant, par un ensemble de mesures convergentes, des conditions générales favorables à l'implantation des entreprises, qu'elles soient industrielles ou de services. Dans ces politiques de promotion de l'attractivité des sites, la qualité, l'efficience et la réactivité des services publics ont pris une nouvelle importance : elles ne se limitent pas à assurer les conditions et les fonctions nécessaires à la vie collective, selon le concept allemand classique de la Daseinsvorsorge ou du concept français non moins classique du Service public, mais sont devenues des leviers essentiels de la compétitivité d'un espace économique, d'un pays ou d'une région.

Dès lors, peu de pays ont vu leur secteur public échapper au cours des vingt dernières années aux pressions exercées par les politiques de réforme conduites par les Etats, politiques encouragées par ailleurs depuis le début des années 1990 par les autorités de l'Union européenne. Les secteurs ayant un impact plus immédiat perceptible sur 
l'économie du pays ont été les premiers concernés. Les politiques de dérégulation ont ainsi radicalement transformé le contexte réglementaire des (anciens) opérateurs publics évoluant sur des marchés dorénavant concurrentiels à l'échelle européenne ou internationale. Ouverture à la concurrence, dérégulation voire désengagement de l'Etat: ce sont les contours même du secteur public qui changent au fur et à mesure que les pouvoirs publics repensent leur rôle et leurs moyens d'intervention.

En République fédérale d'Allemagne, l'administration publique a constitué au lendemain de la Seconde Guerre mondiale une des assises de la réussite du système économique et social. Bien que la bureaucratie fût un objet constant de critique et de réformes, les experts se sont accordés sur un point : l'administration publique vaut mieux que son image. Il faut attendre les années 90 pour qu'intervienne un changement. Face à des caisses publiques vides et au regard des enjeux particuliers de l'unification, le «déficit de modernisation » des administrations allemandes est apparu au grand jour à l'aune d'autres pays de l'OCDE. De nouveaux problèmes émergent au cours de cette décennie: incertitudes croissantes, complexité des mutations technologiques et économiques dans un contexte de globalisation économique renforcée, signaux annonçant des catastrophes rampantes (dans les domaines de la santé ou de l'environnement par exemple), évolution des attentes des citoyens revendiquant une production flexible et de qualité, dans le secteur public également, et spécifiquement pour l'Allemagne, l'enjeu spécifique (financier également) de l'unification.

Les structures administratives, qualifiées de «système d'irresponsabilité organisé », se révèlent inflationnistes. La crise financière déclenche un mouvement de modernisation de l'administration qui promet d'apporter des réponses idoines à trois problèmes essentiels :

- Comment piloter des sociétés développées face aux enjeux posés par une économie qui s'internationalise (globalisation et européanisation) ? La question soulevée ici est celle de la capacité à gouverner des sociétés complexes et différenciées.

- Comment maîtriser la crise financière qui ébranle les institutions publiques, au regard de la pression accrue sur les systèmes sociaux et du coût élevé de l'unification allemande ? On est là en prise avec le problème du financement de l'Etat social moderne.

- Comment tenir compte de l'évolution des valeurs au sein de la société moderne et des aspirations nouvelles des citoyens pour un Etat performant, sur la base de nouvelles modalités de participation et d'engagement ? On touche là à la légitimation de l'action publique.

Face à ces questions, l'Etat et son administration ont atteint leurs limites. La capacité d'innovation semble limitée par des structures de type centralisateur et bureaucratique. Les ressources en personnel sont sous-utilisées, tandis que la participation démocratique des citoyens semble bloquée. Selon les principes du Public Management, l'attractivité de la réforme administrative et de la réforme de l'Etat repose sur l'espoir, scientifiquement démontré, que cette démarche de modernisation est à même de livrer des réponses motivées aux questions énoncées ci-avant. Pour bien comprendre les objectifs de la modernisation de l'administration allemande au cours de 15 dernières années, les concepts sous-jacents et les pratiques actuelles, il faut savoir qu'il ne s'agit pas d'une démarche spécifiquement nationale. Au contraire, la modernisation de l'administration publique a suivi les préceptes du Public Management tel qu'il s'est développé dans les pays anglosaxons, et se réfère à ses grands objectifs : un Etat orienté vers le marché et obéissant à une logique managériale d'une part ; d'autre part, la prépondérance d'une pensée ges- 
tionnaire, également dans les organismes publics. On peut distinguer deux axes de modernisation principaux :

- la redéfinition des missions publiques : les politiques d'externalisation permettent de reconsidérer les limites entre missions accomplies par le secteur public ou le secteur privé. Aux privatisations se substituent de plus en plus de nouvelles formes de diminution de l'activité directe de l'Etat (Outsourcing, Public-Private-Partnership).

- La modernisation interne des organismes publics suivant le concept de neues Steuerungsmodell (nouveau modèle de pilotage) et l'introduction de nouvelles structures au sein des services municipaux (guichets uniques) : le neues Steuerungsmodell est la variante allemande de New Public Management. Son objectif est de transformer une organisation centralisée, fondée sur un fort degré de division du travail et très hiérarchisée en une organisation axée sur les produits (prestations) et structurée en unités bénéficiant d'une large autonomie - dans le cadre de conventions d'objectifs conclues avec les acteurs politiques, les responsables administratifs et les services - et décentralisées. La modernisation interne se réfère donc à la triade suivante : rentabilité, écoute du client et écoute des collaborateurs.

Le concept de guichet unique, adopté aujourd'hui par les deux tiers des communes allemandes, s'articule autour de trois principes :

- décentralisation : c'est à l'administration d'aller vers le citoyen et non l'inverse. C'est la raison pour laquelle les communes installent dans la mesure du possible dans chaque quartier des structures de type guichet unique.

- intégration des tâches: chacun assume les compétences pour l'ensemble des tâches, le guichet unique devant pouvoir proposer une large palette de services.

- participation des employés selon un principe d'auto-organisation. Les salariés disposent de possibilités de participation assez larges, la structure hiérarchique est reconsidérée au bénéfice d'équipes de travail.

En Allemagne, c'est donc l'heure du bilan. Il laisse apparaître un écart assez marqué entre, d'une part, les objectifs et démarches de modernisation du Public Management, et de l'autre, les modifications effectives dans la pratique des administrations. Dans le système allemand caractérisé par différents échelons administratifs, pratiques et avancées en termes de réforme se révèlent donc très disparates selon l'échelon considéré. L'administration du Bund fait ainsi figure de lanterne rouge de la modernisation administrative allemande. Employant 315000 agents soit 6,7\% des effectifs publics, elle a surtout dû gérer au cours des années 90 le déménagement des services de l'ancienne capitale fédérale de Bonn à Berlin. La modernisation de l'administration se situe là essentiellement au niveau de la «communication » et de la discussion de nouveaux concepts allant de « l'Etat svelte » à «l'Etat instigateur », pour revenir récemment à celui de « réduction de la bureaucratie ». Sur le plan des institutions, les transformations majeures ont été le fait des politiques de privatisation. Au début des années 90, la Deutsche Bundesbahn (Deutsche Bahn AG depuis le 1er janvier 1994) et la Deutsche Bundespost (dorénavant Postdienst, Postbank et Telekom) ont été privatisées, sans que leurs administrations internes soient notablement transformées pour autant (sur l'état actuel des réformes, voir Jann 2004 : pp. 100 sq.).

A l'échelon des Länder, la modernisation des administrations offre un caractère plus confus. En théorie, les Länder jouent un rôle clé dans ce domaine. Selon la structure d'organisation de l'administration en République fédérale d'Allemagne, c'est en effet à eux que revient l'exécution de la majorité des lois fédérales. Et ce sont également les 
Länder qui fixent le cadre général de la modernisation des administrations communales (par le contrôle administratif exercé sur les communes, la promulgation des codes municipaux et les réglementations relatives aux procédures). Dans la pratique, certains Länder ont fait figure de pionniers au cours des dix dernières années. Le SchleswigHolstein ou encore la Hesse ont introduit de nouveaux modèles de pilotage et ainsi restructuré l'administration de leurs ministères. Mais la pratique montre aussi que la structure initiale d'une organisation hiérarchique ou encore la répartition des tâches entre politique et administration ne jouent qu'un rôle mineur dans le processus de réforme. Même la récente réforme du fédéralisme ne modifie pas fondamentalement ce tableau; si les compétences du Bund et des Länder sont modifiées dans certains domaines, la relation entre modernisation de l'administration et organisation fédérale de l'Etat n'est toutefois pas au centre du débat sur le fédéralisme.

C'est au niveau communal que la nécessité des réformes a été plus fortement ressentie. En externalisant certaines prestations, en introduisant des modèles de pilotage de l'administration et les guichets uniques, les communes ont fait avancer les concepts. On peut distinguer deux phases : dans un premier temps (du début des années 1990 à 1996/97), le modèle de référence était « de l'administration à l'entreprise de services ». Dans un second temps (depuis 1997), le concept de la modernisation évolue : «de l'entreprise de services à la 'commune citoyenne' » (Bürgerkommune). Le citoyen participe à la production de certaines prestations administratives et à l'organisation des affaires publiques et est impliqué dans de nouvelles formes de coopération. Par exemple, les citoyens assument des fonctions de surveillance dans les piscines publiques, le nettoyage des rues et des parcs, les parents d'élèves rénovent les écoles, les enseignants et les universités entretiennent les universités et prennent ainsi en charge des tâches qui étaient à l'origine du ressort de l'administration. Cette évolution est ambivalente. Les conséquences restent ouvertes, avec notamment le risque de générer une division du travail et des coûts non rémunérée entre les organismes publics et les citoyens, mais aussi l'espoir de susciter un renforcement de la démocratie locale. Les nouvelles formes de participation, en lien avec les modes plébiscitaires de démocratie locale et de processus de représentation (élections) aujourd'hui établis dans l'ensemble des Länder allemands (consultation, référendum), constituent dorénavant le troisième pilier de la démocratie : la démocratie coopérative (Holtkamp / Bogumil / Kißler, 2006).

Les effets de la modernisation des administrations communales se révèlent décevants à l'aune du New Public Management (NPM). Une évaluation nationale de la modernisation des administrations montre que les réformes au niveau communal se réfèrent étonnamment peu au concept de New Public Management (seulement 16,2 \% des communes ayant réformé leur administration) et révèle contre toute attente une forte tendance à la re-centralisation, au ré-établissement des hiérarchies, et au retour aux principes originaux du Public Management. La modernisation à l'aune du NPM n'a pas réduit la pression financière. Les communes sombrent toujours sous le poids d'une dette qui dépasse 11,5 mrd $€$ pour les 12630 communes allemandes. La réforme enregistre pourtant aussi des résultats positifs. L'évaluation révèle des améliorations sensibles dans les relations entre les communes et leur environnement extérieur: elles sont ainsi plus ouvertes aux attentes des citoyens-clients qu'il y a dix ans (Bogumil/Grohs/Kuhlmann, 2006).

Ce résultat s'avère d'autant plus étonnant que la satisfaction des besoins du client et l'ouverture de l'administration sur les citoyens dépend moins de la transformation des structures que d'une évolution de la culture administrative et en particulier des agents de 
l'administration eux-mêmes. Selon Gerhard Banner, l'ancien président de la Кommunale Gemeinschaftsstelle (KGSt), «sans le personnel, cela ne fonctionne pas »: les agents et leurs représentants (les Personalräte) sont les acteurs de la modernisation, mais sont également les premiers concernés par les mesures de réforme. Motivation, qualification mais surtout participation des agents au processus de modernisation constituent le véritable « talon d'Achille» de la réforme dans un contexte de pression sur les coûts et de diktat des impératifs économiques. D'une part, on est face à l'opportunité de considérer le personnel comme une ressource stratégique: heure de gloire d'une institution que l'on cherche désespérément dans les administrations traditionnelles, à savoir la gestion du personnel. Lorsque, dans les années 90, de nouveaux principes inspirés du management des ressources humaines viennent balayer le mode de gestion du personnel dans les municipalités allemandes, il semble alors évident que le management est lui aussi fils de la crise budgétaire. La crise permet de faire du développement, de la motivation et de la gestion des personnels une activité transversale, considérée comme un enjeu stratégique. Mais elle comporte aussi le risque que cette reconnaissance de la " valeur » humaine des collaborateurs ne joue aucun rôle dans la pratique. La contrainte financière a souvent asphyxié les démarches de gestion du personnel. L'investissement dans le recrutement, le développement et l'utilisation optimale du facteur humain reste insuffisants dans les administrations publiques, en tout cas sur le plan financier. Le personnel est considéré avant tout comme un facteur de coût devant faire dès lors l'objet de politiques de réduction des effectifs (voir la contribution de Sabine Kuhlmann pour les données chiffrées les plus récentes). Le management se réduit à sa fonction de soutien aux mesures de rationalisation, conduisant à un écart douloureux entre exigences de rationalisation d'un côté et optimisation de l'utilisation de ressources humaines de l'autre. Gestion et développement des ressources humaines se retrouvent alors en terrain miné. Politiques de rationalisation et pression sur les coûts viennent renforcer ce conflit d'intérêt.

Les personnels témoignent d'une lassitude vis-à-vis des réformes. Plus de la moitié des salariés du secteur public expriment aujourd'hui leur scepticisme, voire rejettent la politique de modernisation de l'administration. La grève qui a paralysé l'Allemagne durant douze semaines au printemps 2006 - la plus longue de l'histoire de la République fédérale - traduit un message sans équivoque. La modernisation des administrations publiques polarise les personnels en deux camps : quelques gagnants et de nombreux perdants, la majorité des personnels semblant se considérer du côté des perdants. Les employeurs publics n'ont, pour l'heure, pas indiqué comment ils entendent corriger ces constats relevant des pratiques de modernisation engagées jusqu'à ce jour et comment ils comptent venir à bout des conséquences négatives qui obèrent l'avenir du service public.

En France aussi, les critiques sont récurrentes face à un secteur public traditionnellement jugé peu efficient, aux procédures routinières, incapable de se réformer autrement que par la contrainte externe, surdimensionné et coûteux. Mais paradoxalement, les enquêtes d'opinion réalisées à intervalle régulier insistent sur l'attachement d'une majorité de la population au «service public » et, ce faisant, à une confirmation de ses missions. De même, le débat s'amplifie sur le maintien de la présence des services publics dans les zones rurales, nécessaire d'un point de vue d'aménagement du territoire et dans la logique républicaine d'homogénéité des conditions de vie, mais coûteux et en défaveur des zones urbaines nécessiteuses des services de l'Etat. 
De fait, la sphère publique a tout d'abord connu après la guerre une longue phase d'expansion soutenue qui s'est prolongée jusqu'à la première moitié des années 80 avec la reprise des politiques publiques de nationalisation dans certains secteurs clés de l'économie et la mise en place des importantes réformes de décentralisation. Dans le même temps, la fonction publique est unifiée et de nouvelles catégories de personnels (par exemple dans le domaine de la recherche) intègrent ce statut, contribuant ainsi à son expansion.

En retour, les deux principales vagues de libéralisation et de privatisation qui ont suivi (1986-1988, puis depuis 1993) ont eu pour effet de réduire fortement l'influence directe de l'Etat dans l'économie. Encouragées par les politiques européennes de renforcement du marché intérieur, ces mesures ont dans un premier temps concerné la quasi-totalité des entreprises et établissements publics et commerciaux du secteur marchand, pour s'étendre progressivement dans les années 90 aux services d'intérêt général. Ouverture à la concurrence et suppression des monopoles d'Etat, restructuration des opérateurs historiques et nouvelles exigences d'adaptation aux besoins des marchés et de la clientèle, pression des marchés sur les coûts de fonctionnement ont donc conduit, pour les entreprises de ces secteurs, à évoluer dans un contexte socioéconomique profondément modifié, contribuant en retour au sein des organisations à substituer à la logique de service public une culture interne de type managérial.

Dans le même temps, et dans le contexte de la politique de convergence des économies européennes (critères du pacte de stabilité et de croissance relatifs aux déficits publics) et d'une forte dégradation des équilibres budgétaires (en Allemagne et en France notamment), des politiques de modernisation des administrations publiques sont menées dans la plupart des pays européens, avec une intensité et une efficacité variables, avec le retour des politiques de rigueur budgétaire au regard du niveau de la dette publique cumulée. En France, différentes démarches sont relayées dans les administrations et entreprises publiques pour forcer la modernisation de la sphère publique, comme l'approche par la qualité du service rendu à l'usager, les projets de service, l'administration électronique depuis la fin des années 90 ou plus récemment l'essor des audits de modernisation ministériels radiographiant les dépenses de l'Etat, etc. Mais bien souvent, il s'agit d'expériences en ordre dispersé, avec une multiplication des outils sans vision globale ni de véritable suivi. Dès lors, une certaine désillusion finit par gagner et transformer la réforme de l'Etat en véritable serpent de mer de la vie politique.

Aujourd'hui pourtant, il semble que les programmes pluriannuels de modernisation, engagés dans les années 90 , commencent à porter leurs premiers fruits. Le message que souhaitent faire passer les partisans de la réforme est que la diminution des coûts de la fonction publique doit passer par une amélioration des procédures et une modernisation des organisations, et pas seulement par des coupes dans les effectifs.

Par ailleurs, on observe un décalage dans les démarches de modernisation entre les différentes fonctions publiques (d'Etat, territoriale et hospitalière). Les territoires dont les compétences se sont progressivement élargies sous l'effet des politiques de décentralisation et de déconcentration des services de l'Etat (transfert de missions de l'administration centrale aux fonctionnaires locaux), ont ainsi développé plus tôt des pratiques managériales parfois innovantes, davantage en phase avec les exigences du terrain. Dès lors, c'est surtout la relance de la réforme institutionnelle, avec la politique de réorganisation de l'Etat et des pouvoirs administratifs ("acte II de la décentralisation »), qui donne aujourd'hui l'occasion à la réforme de l'Etat de franchir une nouvelle étape, 
structurante au plan institutionnel : la Loi constitutionnelle du 28 mars 2003 relative à l'organisation décentralisée de la République, consacre le principe de décentralisation et reconnaît le droit à l'expérimentation. Elle vise ainsi à renforcer les compétences propres des collectivités territoriales, notamment des régions, et se double de la poursuite du mouvement de déconcentration des services de l'Etat: dans la perspective d'accroître la proximité entre l'action publique et le citoyen et d'en renforcer l'efficacité, l'Etat transfère ainsi des missions de l'administration centrale aux fonctionnaires locaux.

A cet égard, la mutation de l'emploi public est bien un aspect central de ce chantier de réorganisation de l'Etat et de répartition des pouvoirs administratifs en France. L'enjeu est double : comment anticiper les besoins en effectifs publics et comment faire évoluer les missions et les modes de travail des agents?

Ce transfert de compétences et d'effectifs s'inscrit par ailleurs dans le contexte d'un vieillissement des effectifs et d'un renouvellement attendu jusqu'en 2020, qui constitue un enjeu de taille pour les trois fonctions publiques : à titre d'exemple $40 \%$ auront pris leur retraite entre 2001 et 2010. Des éléments de réponse sont mis en place avec l'élaboration d'une politique de gestion prévisionnelle des effectifs, des emplois et des compétences, la diversification des modes de recrutement ou encore la mobilité. Par ailleurs, le contexte démographique met en concurrence les recrutements de la fonction publique avec le secteur privé. Là aussi, une opportunité à saisir puisqu'elle peut être l'occasion d'un renouvellement du fonctionnement du secteur public. Cela suppose néanmoins que le secteur public soit en mesure de garantir son attractivité en proposant par exemple aux jeunes diplômés des perspectives de carrière valorisantes. La dimension qualitative de la modernisation joue là un rôle central.

La décentralisation est dès lors vécue comme une opportunité de réorganisation des services, mais sur quel mode ? Comment les changements sont-ils anticipés ? Un vaste chantier de modernisation de la gestion interne et de redéploiement entre les fonctions publiques s'impose et est partiellement engagé. Mais dans un contexte budgétaire tendu - et alors que les dépenses de personnel constituent une part très importante du budget des collectivités territoriales -, la recherche d'une meilleure efficacité et la maitrise des dépenses de personnel constituent des éléments centraux dans la gestion publique, en particulier au niveau des collectivités territoriales, sont susceptibles de limiter la portée du mouvement de modernisation interne.

Enfin, grand chantier de la décennie, la réforme budgétaire adoptée en 2001 par le Parlement et mise en œuvre au 1er janvier 2006 avec la nouvelle Loi organique sur les lois de finance, impose de nouvelles règles du jeu visant à moderniser la gestion publique. Elle annonce des transformations lourdes de conséquences sur un plan quantitatif: réforme du mode d'allocation des financements publics, selon des missions et programmes constituant le cadrage budgétaire de chaque ministère, puis déclinés en actions, en objectifs de service puis répercutés, tout au bas de l'échelle, en objectifs individuels fixés à l'agent, les coûts du personnel apparaissant également dorénavant dans les budgets. Les changements qualitatifs induits n'en sont pas moindres, dans la mesure où la loi génère une modernisation rendue nécessaire de l'organisation du travail avec l'introduction d'une logique de performance et de résultats. La réforme doit entraîner la mise en œuvre d'un fonctionnement par objectifs qui aura des effets sur la manière dont sont gérées les ressources humaines (triple logique d'évaluation, de gestion des compétences et de formation ciblée) et donc en théorie une redistribution justifiée de l'emploi public en fonction des missions. Reste que les effets sont aujourd'hui bien difficiles 
à appréhender mais qu'on se dote d'outils rendant les arbitrages possibles et qu'on s'achemine donc, d'un point de vue de la gestion des ressources humaines, vers une plus forte différenciation au sein de la fonction publique, contre l'avis des syndicats campant sur l'égalité de traitement dans les parcours professionnels (et donc défendant le critère de l'ancienneté).

On est donc aujourd'hui à une époque charnière privilégiant une double approche de la modernisation de l'Etat et de ses services: une logique institutionnelle et une approche technicienne. Les avancées dépendront pour beaucoup de la qualité de management des ressources humaines, qui sera ou non capable de faire accepter les réformes et de les faire vivre.

C'est donc sur les effets des mesures de modernisation pour la gestion de l'emploi et des ressources humaines dans les organisations publiques que le présent ouvrage entend apporter des éclairages, en se basant sur les expériences antérieures mises en œuvre dans les services d'intérêt général, à la confluence entre une logique de marché et les contraintes de l'Etat. Partant d'un constat partagé dans les deux pays d'une pression à la fois économique, sociétale, politique et idéologique exercée sur l'action de l'Etat, la question de l'évolution de l'administration et de l'emploi public est présentée ici dans une approche franco-allemande. Cette approche est motivée par la persistance d'une certaine méconnaissance des réalités politiques et des expériences menées dans le pays partenaire, alors que la France et l'Allemagne constituent respectivement l'un pour l'autre le premier partenaire économique. Cette ignorance est sans doute due au recours toujours systématique à une approche en termes de modèle qui, dans le débat public, conduit à une vision schématisante qui oublie les dynamiques en oeuvre et tend à lisser les contradictions internes inhérentes à tout système. Plus généralement, l'approfondissement du projet européen impose une compréhension juste, basée sur une connaissance précise et actualisée des cultures politiques nationales, à un moment où leur évolution est très rapide. L'intérêt n'est donc pas de chercher dans l'expérience de l'autre un quelconque modèle, mais bien d'enrichir une réflexion propice à stimuler le changement et l'innovation sociale.

L'important aujourd'hui n'est pas en effet de s'interroger sur la validité des stratégies de modernisation engagées depuis plusieurs années et directement liées aux contraintes d'adaptation exercées sur le service public, mais bien d'en mesurer les conséquences en termes de ressources humaines et de management. Toute transformation ne peut être dissociée des effets qu'elle induit et s'il est bon de mettre en évidence les logiques en oeuvre et les raisonnements sous-jacents, il est également indispensable de déterminer les conséquences sociales, jamais homogènes, qui en résultent : car pour tant de gagnants supposés, quels sont les perdants objectifs et subjectifs et comment remédier à cette situation? De cette évaluation résulte bien souvent l'acceptation des changements. Loin d'être prétexte à l'inaction, cette réflexion rappelle que l'introduction de nouvelles règles de gestion va rarement sans déstabiliser l'équilibre existant et peu également conduire à des effets pervers inattendus. D'où l'intérêt, comme le rappellent les témoignages apportés par les contributions des acteurs et praticiens présentés dans l'ouvrage, de la concertation entre acteurs en amont des réformes. L'anti-exemple de nombre d'expériences françaises vient ici renforcer ce jugement. Les nouvelles pratiques en cours dans la sphère publique pourraient dès lors être l'occasion d'un renouvellement des mécanismes de relations sociales. 
La structure en trois parties de l'ouvrage suit la problématique étudiée dans le cadre du projet "Nouveaux modes de régulation sociale dans le secteur public et les services d'intérêt général", mené par le CIRAC de 2004 à 2006. La première partie est consacrée à la question des dérégulations et à l'impact de l'introduction de mécanismes de marché dans les services d'intérêt général. On rappellera ici le cadre européen de régulation des services publics et s'intéressera plus particulièrement aux conséquences sociales du processus de libéralisation en Allemagne, au regard des expériences britanniques et suédoises (Karsten Schneider). La contribution de Rina Bohle Zeller analyse le secteur de l'eau dans lequel les communes allemandes continuent de jouer un rôle primordial mais se trouve confrontées à d'importantes réformes structurelles. Côté français, Pierre Bauby livre une analyse de l'organisation des services d'intérêt général en se fondant sur l'exemple de l'électricité et de l'évolution du secteur dans un contexte de concurrence renforcée, de dérégulation voire de privatisation.

Les contours et les principales caractéristiques de l'emploi public en France (Catherine Zaidman) et en Allemagne (Sabine Kuhlmann) font l'objet de la deuxième partie de cet ouvrage. La question des carrières, des statuts et des formes de recrutement est récurrente mais les velléités de réforme peinent à se concrétiser, en l'absence d'une véritable analyse des missions de l'Etat et des administrations publiques. L'exemple du processus de réforme de la gestion des ressources humaines initié par les autorités du Land allemand de Basse-Saxe (Susanne König) tend à montrer que la large palette d'instruments dont disposent les administrations pour optimiser leurs organisations et leurs modes de fonctionnement ne constitue pas en soi une stratégie de réforme efficace et partagée par les personnels. L'exemple de la Deutsche Telekom AG qui est passée en moins de 15 ans du statut d'administration publique au rôle de Global Player intervenant sur les marchés mondiaux, insiste sur l'importance des procédures de concertation dans l'accompagnement des nouvelles politiques de gestion des ressources humaines et la gestion des conséquences sociales des dérégulations.

Cet aspect est approfondi dans la dernière partie de l'ouvrage. Comment les relations sociales évoluent-elles dans ce contexte? Le témoignage de Philippe Moncourrier analyse l'exemple des relations sociales au sein de la RATP, entreprise considérée, il y a dix ans à peine, comme irréformable et bloquée par des constellations internes de pouvoir. Le rôle et la place de la concertation dans le processus de modernisation, ainsi que l'expérience de co-management des réformes par les représentants du personnel au sein d'une administration municipale de Rhénanie du Nord-Westphalie sont l'objet de la contribution de Hermann Hibbeler. Pour conclure cet ouvrage, Marie-Claude Kervella propose un diagnostic du cadre de concertation dans la fonction publique française et plaide pour une transformation des règles du dialogue social.

\section{Indications Bibliographiques}

Bogumil J., Grohs S., KuHLmanN S., «Ergebnisse und Wirkungen kommunaler Verwaltungsmodernisierung in Deutschland - Eine Evaluation nach 10 Jahren Praxiserfahrung», in : BogUMIL J., JANN W., NullmeIER F. (dir.), : Politik und Verwaltung, PVS-numéro spécial 37/2006

JANN W., Status-Report Verwaltungsreform. Eine Zwischenbilanz nach 10 Jahren, Berlin, 2004

Ministère de la Fonction publique, L'Observatoire de l'emploi public. Rapport annuel 2004-2005, Paris, 2006

LE Clainche M. (dir.), Dossier : «Réformes budgétaires et réformes de l'Etat», Revue français d'administration publique $\mathrm{n}^{\circ} 117 / 2006$ 



$$
-1-
$$

\section{DÉRÉGULATION ET MODERNISATION DE L'ADMINISTRATION EN FranCE ET EN ALLEMAGne}





\title{
La régulation des services publics en Europe et ses effets sur la qualité des prestations, l'emploi et les conditions de travail
}

\author{
Karsten SCHNEIDER
}

En accord avec la politique européenne de renforcement de l'ouverture des marchés, une nouvelle vague de libéralisation a été lancée au début des années 1990 en France et en Allemagne, s'appliquant aux services d'intérêt général (désignés en Allemagne par le concept de Daseinsvorsorge). La suppression des anciens monopoles et/ou les changements de statut d'anciennes administrations publiques, l'adaptation aux marchés et la priorité donnée aux usagers ainsi que la pression financière ont profondément transformé le paysage socioéconomique pour les entreprises concernées. La contribution ci-dessous présente les conséquences économiques et sociales du processus de libéralisation en Allemagne à la lumière des expériences faites dans deux autres pays: d'une part, la Grande-Bretagne, qui a en quelque sorte endossé un rôle de précurseur en termes de déréglementation et de privatisation des services publics ; d'autre part, la Suède, qui tente de mettre en œuvre un modèle socialdémocrate de mesures de libéralisation sans privatisations, c'est-à-dire en préservant le rôle important du secteur public dans l'ensemble de l'économie.

La Commission européenne définit les services relevant des pouvoirs publics comme des «services d'intérêt général » et "services d'intérêt économique général », sans grande distinction. L'Allemagne et la France parlent respectivement de «Daseinsvorsorge » (pourvoir aux besoins fondamentaux) et de service public, ce qui, au-delà de la simple différence de désignation formelle traduit des définitions matériellement différentes des missions publiques. La définition formelle de la Commission est le signe extérieur d'une mise en cause de la légitimité des services publics.

Il convient dans ce contexte de décrire les répercussions de l'activité (économique) des pouvoirs publics et d'évaluer les expériences de transformation du statut juridique («privatisation formelle »), de cession de propriété ("privatisation matérielle ») et de renforcement de la concurrence (libéralisation) avec l'illustration des situations britannique, suédoise et allemande. Nous comparerons ensuite les conséquences respectives, dans ces trois pays, de la libéralisation et de la privatisation dans les secteurs des postes, de l'électricité, des télécommunications, des services des eaux, et dans celui des chemins de fer (la présente étude empirique propose une synthèse; le détail se trouve dans Lippert, 2005 et Kohlmorgen Schneider, 2004).

La question est de savoir comment, grâce à des outils de pilotage appropriés, obtenir dans le cadre des changements de statut et surtout des transferts de propriété, certains effets souhaités par l'État, en matière de sécurité d'approvisionnement par exemple. 


\section{La Grande-Bretagne : le modèle libéral}

La Grande-Bretagne a été le premier État européen à entreprendre à grande échelle une privatisation des services publics. Sous le gouvernement Thatcher, à partir de 1980, pratiquement tous les services publics furent privatisés. Jusqu'en 1993, British Telecom fut privatisé en totalité. Dans le secteur énergétique, ce processus était achevé en 1990, les services des eaux ayant été entièrement cédés dès 1989. Les chemins de fer ne furent privatisés en totalité qu'en 1997 ; pour ce qui est de la poste britannique, en revanche, aucune privatisation, même à venir, n'est à prévoir. La recherche en science politique qualifie ce très large processus de privatisation de "glissement de terrain institutionnel ». L'exception du British Post s'explique par le degré élevé de syndicalisation : la vente a ainsi pu être empêchée.

Le modèle britannique a ceci de particulier qu'il y a eu transfert de propriété, autrement dit privatisation matérielle, mais qu'aucune ouverture des marchés n'a été réalisée : de 1984 à 1990, le secteur des télécoms britanniques était structuré de façon duopolistique. En 1991, le marché fut davantage ouvert, mais British Telecom en détient encore aujourd'hui $75 \%$ des parts. Il n'y a pas eu ouverture du marché dans le secteur des eaux; celui de l'électricité est caractérisé par ce que l'on appelle un marché « artificiel». La poste ne s'est mise à attribuer des concessions qu'en 2003. Dans ce secteur, le degré d'ouverture du marché est estimé à $22 \%$. Les seuls exemples positifs d'une concurrence effective en Grande-Bretagne sont le secteur des colis postaux et le transport ferroviaire régional.

De ce fait, le modèle britannique fait l'objet d'une évaluation critique dans les travaux de recherche, car c'est la concurrence et non la forme de propriété qui constitue un critère déterminant de performance économique.

La Grande-Bretagne ayant proclamé le désengagement de l'État dans la production des services publics, on pourrait s'étonner que celui-ci ait encore beaucoup d'activités dans ce domaine. Les pouvoirs publics s'efforcent, par le biais d'organes de régulation, de contrôler la production privée des services publics. Le modèle britannique est surtout caractérisé par la séparation des activités (Unbundling) : les infrastructures sont détenues par l'État, mais la production et la distribution des services sont gérées par des entreprises privées. À la suite d'énormes problèmes de qualité, cette structure n'a été établie qu'en 2002 dans les secteurs des services d'eaux et des chemins de fer. Les infrastructures autrefois privatisées, donc le réseau, sont repassées dans le secteur public. La séparation des activités peut être considérée comme une caractéristique marquante du modèle britannique ; elle est le signe extérieur et la condition d'une intervention à des fins de régulation.

En Grande-Bretagne, les institutions de régulation de tous les secteurs sont fortes et indépendantes, leur financement étant surtout assuré par les attributions de licences. Leur mission est de veiller à la qualité, et d'assurer la régulation des prix ex-ante. La régulation des prix se manifeste, notamment sur le marché des télécoms et de l'énergie, sous forme de plafonnement des prix (Price Caps). Dans le secteur ferroviaire, les autorités de régulation fixent le montant des redevances d'utilisation du réseau. Ces mécanismes « artificiels » requièrent globalement un appareil d'État fort et développé, ce qui peut être considéré, pour le moins, comme paradoxal par rapport à l'objectif poursuivi : la réduction de l'influence de l'État. Les fortes interventions régulatrices attestent d'un mauvais équilibre entre l'offre et la demande. 


\section{La Suède : le modèle social-démocrate}

Les modifications structurelles des services publics n'ont quasiment pas affecté les spécificités du modèle suédois. Le secteur public est encore vaste et influent; la structure publique reste marquée par le corporatisme. Au lieu d'opter comme la Grande-Bretagne pour le changement de statut juridique et, plus encore, pour la privatisation matérielle, la Suède s'est efforcée d'exploiter les effets positifs de l'ouverture à la concurrence : concrètement, seule la société de télécommunications a été vendue à $30 \%$, avec une privatisation prévue à $49 \%$ maximum. Dans le domaine de l'énergie et des postes, il n'y a jusqu'à présent pas eu de privatisation. Dans le secteur de l'eau, $3 \%$ des entreprises sont privatisées. Sur six sociétés ferroviaires, trois sont détenues par des exploitants privés, et trois restent en régie de l'Etat. À la différence de la GrandeBretagne, le rôle considérable des pouvoirs publics, se traduit donc par le maintien de la propriété de l'Etat.

Mais, depuis le milieu des années 1980, les différents secteurs ont été ouverts au marché. Depuis 1996, presque tous ont été libéralisés. Celui des eaux fait toutefois exception. En 1991, des marges de manœuvre ont été accordées aux Régions pour des appels d'offres, mais, comme la qualité et les prix de l'eau en Suède étaient déjà très bons, ces libertés n’ont pas été exploitées. Globalement, le modèle suédois est qualifié de «concurrence régulée ». Malgré une position forte des entreprises publiques, la concurrence a été introduite dans tous les secteurs.

En raison de cette position solide (et donc de l'influence des pouvoirs publics qui en résulte), les institutions de régulation n'ont pas eu les mêmes prérogatives qu'en GrandeBretagne. Celles qui ont été créées sont davantage la conséquence d'initiatives européennes que le résultat d'une nécessité de réguler. Leur rôle consiste surtout à analyser le marché, bien que des instruments d'intervention aient été créés - justement sous l'influence de l'Europe. La séparation des activités joue un rôle moins important : dans le secteur ferroviaire, la dissociation du réseau et de son exploitation a été initiée en 1988 et renforcée en 2001. Dans le secteur électrique, les opérateurs doivent se conformer à un cahier des charges fixé par l'Etat. Seules les postes connaissent le plafonnement des prix ; dans les autres secteurs, il n'existe aucune forme de régulation des prix. Globalement, les autorités de régulation sont relativement dépendantes, elles ne disposent pas de recettes propres; seul dans le secteur des télécoms l'instance de régulation présente une plus grande indépendance, son financement étant assuré par l'attribution de licences.

\section{L'Allemagne : un modèle mixte}

À la différence de la Suède et de la Grande-Bretagne, qui ont choisi leurs modalités respectives de libéralisation de manière autonome, c'est-à-dire de leur propre initiative, l'Allemagne a procédé à l'ouverture des différents secteurs en réaction aux directives de la Commission européenne. La société Deutsche Telekom n'est maintenant plus détenue par l'État qu'à $48 \%$; la Deutsche Post reste publique à $69 \%$, tandis que les chemins de fer le restent à $100 \%$; on peut estimer que l'approvisionnement en eau et le traitement des eaux usées sont aux mains des pouvoirs publics à hauteur de plus de $80 \%$. Le secteur énergétique est un cas particulier dans la mesure où il n'existe pas de données précises, certains grands distributeurs allemands s'étant développés à partir du niveau 
local/communal. Mais on peut estimer que moins de $25 \%$ de l'approvisionnement en énergie sont la propriété des pouvoirs publics.

En matière d'ouverture des marchés, l'Allemagne est encore plus frileuse. Si des tendances à la libéralisation des télécoms existent depuis 1990, il a fallu attendre 1996 pour que soit introduite l'attribution de licenses d'exploitation du réseau. La Deutsche Post dispose jusqu'en 2007 d'une license exclusive pour les lettres jusqu'à $200 \mathrm{~g}$ et les envois en nombre jusqu'à $50 \mathrm{~g}$. La Deutsche Telekom, de même que la Deutsche Post pour les colis, disposent d'un monopole de fait; dans le secteur de l'électricité, les structures oligopolistiques sont prédominantes.

Le système allemand de régulation est de nature plutôt conservatrice. Il ne présente comparativement presque pas d'obligations de répartition des activités. Le contrôle des prix s'effectue ex-post, les incitations économiques sont rares. Seul le secteur des postes connaît des prix plafonnés, et celui des télécoms des contrôles ex-ante. La Deutsche Post a en outre l'obligation d'assurer un service de base. Globalement, l'autorité de régulation a peu de pouvoirs. Dans le secteur de l'eau, il n'existe aucun organe de régulation. Dans celui de l'énergie, l'Agence fédérale des réseaux (Bundesnetzagentur, anciennement RegTP, autorité de régulation des postes et télécommunications) vient tout juste d'en être chargée. Cette institution, soumise à l'influence directe du ministère fédéral de l'Économie, peut être qualifiée de faible.

\section{Conséquences économiques et sociales de la libéralisation}

\section{Rapport qualité/prix}

Les partisans d'une libéralisation en attendent des baisses de prix et une amélioration de la qualité, tandis que ses adversaires redoutent avant tout des problèmes d'approvisionnement. Si l'on observe précisément les cinq secteurs évoqués, on obtient un tableau nuancé.

On constate dans le secteur des télécoms des effets plutôt positifs : la qualité s'améliore, et les prix baissent. A l'inverse, on observe dans celui des postes de fréquentes augmentations de prix, surtout en Suède, mais également en Allemagne et en GrandeBretagne. Mais elles sont souvent le corollaire d'améliorations de la qualité.

Sur le marché de l'énergie, on constate, surtout en Grande-Bretagne, des baisses de prix et des améliorations importantes de la qualité. En Suède, les prix ont en revanche fortement augmenté tandis que la qualité déclinait ; en Allemagne, la qualité d'approvisionnement est constante et les prix ont légèrement augmenté.

Le secteur de l'eau se caractérise en Grande-Bretagne par d'importantes hausses de prix et une diminution de la qualité. En Allemagne, les hausses de prix sont rares, et le niveau de qualité reste élevé. La Suède présente, comme cela a déjà été dit, un haut niveau de qualité et des prix stables. Et ce, alors que d'importants investissements ont été réalisés.

Dans les transports ferroviaires, on observe en Grande-Bretagne de considérables hausses de prix et une qualité sensiblement inférieure ; en Suède et en Allemagne, les prix ont légèrement diminué, et la qualité s'est nettement ou modérément accrue.

Il ressort, pour résumer, des effets positifs surtout pour les télécoms, tandis que les répercussions sont particulièrement négatives sur les secteurs de l'eau et des chemins de fer britanniques. 


\section{Emploi}

À la suite des mesures de libéralisation, on observe dans tous les secteurs de nettes tendances à la réduction des emplois. Les chiffres disponibles sont toutefois de qualité très variable. On ne trouve de données fiables que pour les télécoms, les postes et les chemins de fer. Mais, même dans ces secteurs, on se heurte à une limite : pour les nouveaux opérateurs privés concurrençant des entreprises autrefois ou bien encore actuellement publiques, les données ne sont pas toujours d'une qualité suffisante.

Pour le secteur des télécoms, on n'observe globalement pas d'évolution négative de l'emploi, mais pas non plus d'augmentation des effectifs, tandis que pour les postes, les compressions d'effectifs dans les entreprises autrefois publiques ont également été compensées par des embauches dans les entreprises privées. En Suède, les suppressions d'emploi dans les entreprises (anciennement) publiques représentent $30 \%$ des effectifs, en Allemagne $18 \%$ et en Grande-Bretagne, dans les conditions citées, $2 \%$ seulement. Il faut toutefois avoir à l'esprit que, parmi les emplois créés, beaucoup sont de nature précaire, et que les chiffres relatifs aux effets compensatoires des décisions de recrutement dans le secteur privé reposent largement sur des estimations.

On observe dans le secteur énergétique une forte diminution de l'emploi. En Suède, celle-ci s'élève à $17 \%$, en Allemagne à $23 \%$, en Grande-Bretagne à $29 \%$. Aucune donnée n'est disponible concernant l'impact des entreprises privées sur l'emploi. Entre 1989 et 1999, le secteur de l'eau a perdu 20000 emplois sur 50000 en Grande-Bretagne ; en Allemagne, environ $25 \%$ des postes ont été supprimés entre 1991 et 2001.

Dans les chemins de fer, un tiers des emplois a disparu en Grande-Bretagne, $50 \%$ en Suède. En Allemagne, sur 480000 emplois en 1990, plus de la moitié ont été supprimés jusqu'en 2005, ce phénomène étant toutefois surtout lié aux conséquences de l'unité allemande. Ces coupes n'ont pas été compensées par les entreprises privées, ou bien seulement dans une très faible proportion.

Il apparait globalement que les importantes baisses d'effectifs sont surtout le fait des secteurs ferroviaire et de l'eau, mais que l'impact sur les autres secteurs n'est pas aussi radical. Il faut toutefois prendre en compte la forte augmentation des emplois précaires dans le sillage de la libéralisation.

\section{Conditions de travail}

Dans les secteurs analysés, l'intensité du travail est en augmentation constante. Les temps de travail régulier s'allongent, les heures supplémentaires se multiplient. Les répercussions négatives sur la santé des employés apparaissent par conséquent, le nombre d'accidents du travail augmentant aussi. Les revenus diminuent pour les salariés. La situation des personnes recrutées depuis peu est moins favorable et, pour l'ensemble des personnels, certaines primes versées auparavant disparaissent. Les prestations sociales et la formation continue sont elles aussi beaucoup plus restreintes.

On observe globalement une augmentation des formes précaires de travail et des emplois médiocres ou sous-qualifiés. Les plus touchées par cette évolution sont les femmes, notamment parce que le niveau d'encadrement intermédiaire disparaît ou est fortement réduit. 


\section{Relations sociales}

La libéralisation peut aussi être vue comme une stratégie d'affaiblissement des syndicats, en particulier en Grande-Bretagne, où les conditions de travail font désormais surtout l'objet de négociations individuelles. En Suède, les syndicats, traditionnellement organisés par métiers, envisagent de plus en plus de se regrouper en fédérations organisées par secteur, afin de maintenir leur capacité d'action et de négociation.

\section{La conception européenne des services publics}

La Commission européenne se soustrait à la tâche de définir positivement ce que doivent être selon elle les prestations de service public ou les «prestations d'intérêt (économique) général ». Cela ne l'empêche toutefois pas d'intervenir à des fins de régulation. Avec son projet de directive sur les services, elle a tenté d'introduire - y compris pour la majorité des services publics ${ }^{1}$ - le principe du " pays d'origine », un instrument permettant de faire sauter les minima en termes de salaires et de conditions de travail (voir Lorenz 2005 ; Böhret et al., 2005). Il faut donc saluer la reformulation de ce principe par le Parlement européen. Notons cependant que la Commission se soustrait à sa mission d'harmoniser les prestations de service public en Europe. Le compromis formulé au Parlement européen remplace le principe du pays d'origine par l'obligation active des États membres de supprimer les barrières que représentent par exemple certaines normes.

On pourrait supposer, relativement à l'arrêt du 11 janvier 2005 (Az. C-26/03) de la Cour de Justice de l'Union Européenne sur l'attribution de contrats, que les partenariats public-privé (PPP) vont perdre de l'importance (voir à ce propos Nagel et al., 2005). Mais le Livre vert sur les partenariats public-privé présenté en avril 2004 ne porte pas uniquement sur cette question. Il comporte notamment une obligation de mise en concurrence, selon laquelle toute activité économique devra faire l'objet d'un contrat de concession. Le plus problématique dans ce contexte, c'est que la Commission européenne n'accepte de reconnaître l'activité non économique des municipalités que si la société ou l'entreprise concernée intervient pour les besoins propres de la ville, c'est-àdire si les usagers ne sont pas les destinataires des services.

On omet largement le fait que, selon la volonté du Livre blanc sur les services d'intérêt général (mai 2004), les services sociaux devraient, eux aussi, être «modernisés », ce qui vaut par exemple pour le secteur de la santé. Dans ce domaine, les États d'Europe centrale et de l'Est ont intérêt à maintenir les coûts salariaux annexes à un niveau bas. Or, ces Etats pèsent actuellement de toute évidence sur le débat au Conseil européen.

L'action de la Commission européenne va donc actuellement dans le sens d'une plus grande tendance à la libéralisation des services publics, sans prendre en compte certaines répercussions possibles telles qu'elles ont été éprouvées de manière plutôt douloureuse en Grande-Bretagne, ou plutôt positive en Suède.

LES RÉSULTATS EMPIRIQUES indiquent que des pertes de contrôle surviennent déjà dans le cadre des changements de statut des services publics (voir Bremeier et al. 2005

\footnotetext{
${ }^{1}$ Assez tôt dans le processus, la Commission a par exemple exclu l'ensemble du secteur de la santé. Compte tenu de la jurisprudence très restrictive de la CJE en matière d'exceptions, une dérogation n'aurait sans doute pas suffi (voir Lorenz, 2005).
} 
parmi d'autres références). La comparaison Suède/Grande-Bretagne fait en outre apparaître un effet comparativement positif de l'influence directe - c'est-à-dire en cas de propriété de l'État - des pouvoirs publics sur la production des prestations. Il est manifestement plus simple de garantir le respect de normes en particulier sociales et qualitatives dans les entreprises publiques. L'État hiérarchique, régulateur, semble se heurter aux limites de son efficacité, et l'on peut se demander si l'on ne surestime pas depuis toujours le mode de fonctionnement hiérarchique. Même dans le cas d'une production publique propre, un État ne peut agir de manière uniquement hiérarchique ; il doit laisser de plus en plus de place à des approches coopératives. Ceci est manifestement plus difficile dans le cadre d'une mise en avant des autorités publiques de régulation. Il s'avère dans tous les cas qu'un monopole privé pose plus de problèmes qu'un monopole public.

L'action de la Commission européenne fait apparaître une prise en considération insuffisante des aspects sociaux. On peut dès lors se demander si l'échec de la Constitution pourrait avoir aussi des conséquences positives relativement au manque de transparence des procédures, et ne pourrait inciter la Commission à intervenir de manière plus équilibrée.

\section{Traduction de Marie GRAVEY}

\section{Indications bibliographiques}

Böhret C., Grunow D., ZIEKow J., (éd.), Der Vorschlag zu einer Richtlinie des Europäischen Parlaments und des Rates über Dienstleistungen im Binnenmarkt. Regelungsgehalt - Problemfelder - Akteurspositionen, Speyer (Speyerer Forschungsberichte, vol. 241), 2005, http ://192.124.238.222/fbpdf/fb-241.pdf, 2006

Bremeier W., Brinckmann H., Killian W., Schneider K., «Die Bedeutung des Corporate Governance Kodex für kommunale Unternehmen», in : Zeitschrift für öffentliche und gemeinwirtschaftliche Unternehmen, cahier 3, 2005, p. 267-282

KoHLMORgEN L., SCHNEIDER K., «Deregulierung der Wasserversorgung und des Verkehrs im internationalen Vergleich», in : WSI Mitteilungen, cahier 2, 2004, p. 90-95

LIPPERT I., Öffentliche Dienstleistungen unter EU-Einfluss. Liberalisierung - Privatisierung - Restrukturierung - Regulierung (Modernisierung des öffentlichen Sektors, vol. 26), Berlin, 2006

LORENZ F., Der Entwurf einer EU-Dienstleistungsrichtlinie in rechtlicher Hinsicht. Ein Gutachten im Auftrag der Hans-Böckler-Stiftung, Düsseldorf, 2005

NAGEl B., SCHEele U., POLlEM O., «Dienste im allgemeinen wirtschaftlichen Interesse nach Art. 16 des EGVertrages und die Versorgung mit Wasser, Gas und Elektrizität.», Endbericht an die Hans-Böckler-Stiftung, Kassel, 2005 



\title{
Dérégulations et privatisations dans les services publics en France : l'exemple du secteur de l'électricité
}

\author{
Pierre BAUBY
}

\begin{abstract}
Contrairement à l'idée largement diffusée, l'organisation des services d'intérêt général en France est loin du schéma monolithique que véhicule l'expression de "service public à la française ", porteuse de confusion. Résultat d'un long cheminement historique, ce sont en réalité deux modèles très distincts qui se sont développés en France. Même s'ils sont à bien des égards faussés par rapport au modèle théorique et initial sous-jacent, ils continuent aujourd'hui de coexister. Pourtant, quelle que soit la forme organisationnelle, c'est la conception même de services publics nationaux qui est aujourd'hui remise en question par un faisceau de circonstances endogènes et exogènes. Cette contribution dresse un état du cadre et des enjeux économiques et sociaux de la libéralisation et de la dérégulation dans les services d'intérêt général en France, en s'appuyant tout particulièrement sur l'exemple du secteur de l'électricité. Elle rappelle également que les perspectives stratégiques d'évolution des services d'intérêt général restent à ce jour largement ouvertes.
\end{abstract}

Une première question qu'il convient d'éclaircir lorsque l'on parle de services publics est d'ordre terminologique. Dans la tradition française, ce concept recèle toute une série de confusions. On confond ainsi aisément, sous la même appellation de services publics, les services publics administratifs, régaliens et les services publics à caractère industriel et commercial (énergie, transports, communications). La même appellation désigne tout autant des services publics nationaux, dépendants de l'Etat, et des services publics locaux, dépendants des collectivités territoriales et locales (en particulier les communes et regroupements de communes). Troisième confusion majeure : il $\mathrm{y}$ a amalgame entre missions, objectifs, finalités et organisations. Par abus de langage, on présentera ainsi la SNCF comme un service public, alors qu'il s'agit d'une entreprise chargée de certaines missions de service public, dont un grand nombre d'activités ne relèvent pas de missions de service public : la SNCF est ainsi le premier transporteur routier en France. Enfin, on confondra également entreprises publiques et privées. Le mot 'public' de service public laisse en effet entendre que l'on parle d'entreprises à statut public, voire directement d'activités de l'Etat ou des collectivités, alors qu'il existe en France une tradition très ancienne de délégation de gestion de missions de service public à des entreprises privées, à des sociétés d'économie mixte, ou encore, en particulier dans le domaine social, à des associations sans but lucratif. Le terme générique de services publics recouvre en réalité une extrême diversité.

Autre source de confusion : on assimile souvent entreprises publiques et monopoles alors que la situation, autrement plus complexe, autorise des missions de service public dans des situations partielles de concurrence. De même, si la question des statuts 
particuliers des personnels de certains services publics est un enjeu évident des évolutions actuelles, il ne s'agit pas là d'un cas généralisable.

Cette contribution s'appuiera dès lors sur une définition qui insiste sur les objectifs et les finalités et permet ainsi de s'entendre sur une réalité commune à l'ensemble des pays européens, sous des formes et des traditions certes différentes, mais avec ce que l'actuel Traité et le projet de Constitution européenne nomment des valeurs communes. Elle repose sur le fait que, dans tous les pays européens, les autorités publiques nationales, fédérales, régionales ou locales, ont décidé que certaines activités ne peuvent relever du seul droit commun de la concurrence et des règles communes du marché, mais doivent relever aussi de certaines règles particulières, afin de réaliser trois objectifs généraux :

- garantir l'accès de chaque habitant à des biens ou des services essentiels (droit à l'éducation, droit à la santé, aux transports, etc.), chaque individu se voyant aujourd'hui reconnaître dans nos pays européens un certain nombre de droits ;

-assurer des rapports de solidarité et de cohésion économique, sociale et territoriale (on parle en France d'égalité, par attachement à l'égalité républicaine et nationale, mais on mesure bien là les différences avec l'histoire allemande) ;

-prendre en compte le long terme, que l'on désigne aujourd'hui par développement durable, qui exprime la volonté de préparer l'avenir dans l'intérêt des générations futures (dans l'éducation, la santé, les transports, l'énergie, etc.).

Ceci constitue le cœur de ce qu'il conviendra d'appeler, dans cette contribution, services publics ou services d'intérêt général, deux notions que nous assimilerons sans distinction lorsque nous nous référerons aux objectifs (et non aux formes organiques). On peut ainsi assimiler services publics et services d'intérêt général d'un côté, services publics à caractère industriel et commercial et services d'intérêt économique général, cités dans le Traité européen, de l'autre.

Dès lors, on constate que la tendance en France, voire en Europe, à parler de services publics à la française, est porteuse de confusions et d'incompréhensions, dans la mesure où la tradition repose en réalité en France non pas sur un modèle monolithique, mais bien sur deux modèles de gestion. Le premier, que l'on évoque implicitement ou explicitement lorsque l'on parle de services publics à la française, est bien sûr celui de services publics nationaux gérés par les entreprises publiques nationales, avec comme caractéristique essentielle une situation de monopole, la centralisation et le statut des personnels (EDF, SNCF, La Poste, etc.).

Le deuxième modèle est celui d'une gestion déléguée des services publics. Très ancien, il existait déjà sous l'Ancien Régime et est aujourd'hui très présent dans le domaine de l'eau, de l'assainissement, des transports urbains et de beaucoup d'autres activités de service public, de sorte que les entreprises françaises privées de services comptent parmi les leaders mondiaux dans certains secteurs (eau, assainissement). Mais l'un comme l'autre, ces modèles connaissent aujourd'hui des mutations profondes dont il est difficile d'évaluer la portée.

\section{Les services publics nationaux : l'exemple d'EDF}

Nous prendrons ici l'exemple d'EDF en tant qu'idéal-type pour fonder notre réflexion, étant entendu que les entreprises ont chacune suivi des évolutions quelque peu différentes (par exemple, France Télécom et La Poste, en tant qu'anciennes administrations 
d'Etat et non entreprises publiques comme EDF) et rappellerons brièvement l'histoire de l'entreprise, démarche indispensable si l'on veut bien saisir l'ampleur et la portée des mutations présentes.

Dans tous les pays, l'arrivée de l'électricité dans l'économie et la société se produit de manière identique : à l'origine, elle apparaît sous la forme d'une source d'énergie décentralisée, sur la base d'initiatives individuelles, privées, locales et concurrentielles. A grands traits, le développement technique produit les premiers réseaux d'interconnexion, d'échanges et de distribution qui vont être utilisés pour l'éclairage public. De la concurrence émerge, vers la fin du XIXe siècle/début XXe siècle, la supériorité du courant alternatif et une première standardisation, qui naît donc du développement économique et technologique. Une première loi définit en 1906 en France le régime de concession de distribution publique. Les réseaux électriques occupent le domaine public et pour éviter l'anarchie dans l'utilisation du domaine public, les autorités d'alors créent un régime de concession de distribution publique par une loi nationale autorisant l'attribution d'un monopole de distribution à un acteur, moyennant en contrepartie l'obligation de respecter certaines règles sur le produit, le service, le tarif. En échange de ce monopole, accordé pour l'essentiel à des entreprises privées, on fixe donc des règles et un certain nombre de contraintes et d'obligations, qui seront dès 1906 à la base du régime de concession de distribution publique.

En parallèle, le progrès technique va continuer de progresser très rapidement (gains de productivité, mise au point du transport à longue distance qui permet de passer progressivement de réseaux locaux à des réseaux régionaux, nationaux et aujourd'hui européens). Du progrès technique vont naître des systèmes intégrant les trois fonctions de production-transport-distribution jusqu'au client final. Dans ce contexte, les collectivités locales continuent de jouer un très grand rôle au début du XXe siècle. Des concessions de distribution sur de longues périodes sont alors mises en place. A l'époque et dans ses prémisses, l'électricité est donc un service public local avec un suivi de l'Etat.

Des années 1920 aux années 1940 va émerger un système de production-transportdistribution intégré progressivement au plan national, avec quelques échanges avec les pays voisins. Dans le même temps, le progrès technique, d'une part, les rendements croissants, d'autre part, génèrent des concentrations, quelques grosses entreprises et de nombreuses petites entreprises se constituant dans l'entre-deux-guerres. La législation va alors définir progressivement un service public à dimension nationale. Les collectivités locales continuent certes à accorder des concessions de distribution mais, dans le même temps, on constate l'émergence de préoccupations nationales: l'idée de considérer l'électricité comme un bien public avec obligation de couverture et de desserte émerge progressivement alors même que se constitue un oligopole de quelques grandes compagnies privées. Celles-ci font du reste l'objet d'importants débats et de luttes sociales dans la France des années 30 et sont la cible de trois reproches essentiels : le premier est de ne desservir que les zones les plus rentables. L'électrification complète de la France et des zones rurales, qui n'interviendra qu'après la guerre, donne alors lieu à de véritables batailles. On leur reproche également de profiter de leur situation de monopoles locaux ou territoriaux pour une durée de temps déterminée en pratiquant des tarifs trop élevés. Progressivement, l'Etat va donc intervenir dans ce débat sur les tarifs et tenter, face à la demande sociale, de plafonner les tarifs, même si on est encore loin de la péréquation qui n'interviendra que bien après. 
Enfin, troisième grief, les compagnies privées sont accusées de ne pas suffisamment investir : cela donnera lieu à un vaste débat dans la même période sur l'exploitation du potentiel hydraulique du Massif central puis des Alpes, investissements réputés très lourds et à long temps de retour sur investissement. Face aux réticences, l'Etat définira juste avant la guerre le programme des trois milliards destiné à lancer le développement hydraulique, en particulier dans les Alpes. Bien que celui-ci ne pourra finalement être mis en œuvre qu'après la guerre, cette démarche démontre la préoccupation de l'Etat amené à intervenir pour organiser et réguler une concurrence oligopolistique.

\section{La constitution d'un « modèle EDF »}

EDF est créée en 1946 par la nationalisation des entreprises privées. Il existait certes également des régies municipales là où certaines communes géraient elles-mêmes leurs services de distribution, voire parfois de production, de l'électricité. Mais celles-ci étant déjà de statut public, elles ne seront pas intégrées à EDF et nationalisées dans le compromis de la Loi de 1946. Certaines continuent d'ailleurs d'exister aujourd'hui, même si leur rôle est largement marginal dans le secteur électrique français. La nationalisation, en 1946, des compagnies privées se révèle être la résultante d'une triple conjonction : d'une part des débats et batailles des années 30 avec les tentatives de régulation des oligopoles privés; d'autre part, le contexte de reconstruction dans l'aprèsguerre, alors que les besoins d'investissement sont tels que l'Etat est amené à jouer un rôle moteur pour générer et organiser la reconstruction. Enfin, le contexte sociopolitique de l'après-guerre (issu du Conseil national de la Résistance) consacre le consensus entre les Gaullistes, les Socialistes et les Communistes. Dans le secteur extrêmement syndicalisé de l'électricité, la $\mathrm{CGT}^{1}$ représente, dans la Résistance et dans l'Après-guerre, l'essentiel des forces sociales syndicalisées du secteur. Marcel Paul, ministre de l'Industrie du Général de Gaulle, œuvre pour que la Fédération CGT de l'énergie puisse jouer un rôle majeur. Cette conjonction entre les enjeux technicoéconomiques des années 30, le contexte politique et la volonté de reconstruction finissent donc par converger de telle sorte que la nationalisation, considérée comme seule option possible, est votée à la quasi unanimité et fait l'objet en France d'un large consensus. De ce point de vue, l'exemple britannique sera à l'époque assez semblable, tandis que les histoires française et allemande, relativement parallèles jusqu'à la seconde guerre mondiale, vont ensuite diverger dans les formes d'organisation.

La nationalisation apparaît donc à la fois comme un aboutissement de processus antérieurs et un élément de rupture, puisque l'on fait basculer dans le giron de l'Etat des compagnies privées. C'est ce compromis entre rupture et continuité profonde qui va créer un «modèle EDF », modèle technico-économique, politique et social qui, fait exceptionnel, va, globalement, rester stable pendant près de 50 ans.

Ce «modèle EDF» est caractérisé par une cohérence et une intégration tout à fait particulières : c'est le cadre français national. Bien qu'EDF développe quelques échanges avec les pays voisins, son cadre de définition et de conception reste la France et le niveau national. Sa deuxième caractéristique est celle d'un quasi monopole, total pour le transport et à hauteur de $95 \%$ de la production et de la distribution d'électricité en France. EDF est par ailleurs un établissement public à caractère industriel et commercial (EPIC) dont $100 \%$ des capitaux sont restés publics jusqu'à l'ouverture partielle

\footnotetext{
${ }^{1}$ Confédération Générale du Travail
} 
du capital (15\%) opérée à l'automne 2005. Ses missions et les objectifs de service public sont alors tellement évidents que la loi de nationalisation de 1946 les évoque à peine. C'est en même temps un système centralisé qui va se mettre en place et se constituer progressivement, pour bénéficier des avantages de l'intégration productiontransport-distribution. Ce modèle repose sur le principe traditionnel d'égalité dans l'universalité de l'accès. Sur un plan organisationnel, il a pour particularité d'entretenir un jeu reposant sur trois acteurs majeurs : l'Etat, les dirigeants de l'entreprise et un syndicalisme incarné très majoritairement par la CGT. Il s'agit par ailleurs d'un modèle centralisé et hiérarchisé, défini au niveau de l'Etat. Cette caractéristique n'était toutefois pas explicitement présente dans la loi de 1946, qui prévoyait, sans les définir, des structures et des modes d'organisation beaucoup plus décentralisés que ce qui va finalement se mettre en place. Mais la conjonction des trois acteurs va pousser à un modèle très centralisé dans lequel chaque acteur s'y retrouve. Avec toutefois une double perversion : d'un côté, l'entreprise et ses dirigeants concentrent le monopole d'expertise sur l'énergie en France, puisque ce sont pour l'essentiel les grands corps techniques et EDF qui vont définir la politique énergétique de la France. On peut donc dans une certaine mesure parler à ce propos de capture du régulateur par l'opérateur. Mais en parallèle, la tutelle de l'Etat (c'est le terme officiellement employé pour désigner le rapport de l'Etat avec une entreprise de service public) se rattrape par une tutelle administrative tatillonne, voire souvent des instrumentalisations d'EDF et du service public pour d'autres buts que des missions de service public.

Les usagers qui étaient définis dans la Loi de 1946 comme un des trois acteurs majeurs (conseil d'administration tripartite avec l'Etat, les personnels, les usagers), sont donc progressivement relégués au rang d'objets. Les collectivités territoriales, détentrices du pouvoir de concession des réseaux de distribution en particulier, sont, dans la réalité, marginalisées, puisqu'elles peuvent certes concéder, mais obligatoirement à EDF : le rapport de force est dès lors forcément inégal entre EDF, monopole national et les 36000 communes que compte la France. Enfin, les personnels et les syndicats vont être progressivement écartés des décisions stratégiques malgré le maintien de ce jeu à trois au sommet. C'est sur cette base que se constitue, se développe et se solidifie ce modèle dans les années 50, 60 et 70 .

\section{L'impact de la libéralisation sur le « modèle EDF ॥}

Comment et pourquoi intervient la libéralisation de ce point de vue ? La première dimension est celle de l'intégration européenne qui va conduire à l'introduction de la concurrence dans le secteur électrique de chaque pays, avec son mode d'organisation national traditionnel, dans la mesure où la constitution d'un marché européen implique une ouverture des frontières et, de ce fait, une ouverture des monopoles nationaux ou locaux à la concurrence. La deuxième dimension de la politique européenne de libéralisation est que l'aiguillon de la concurrence va pousser à davantage d'efficacité là où les structures monopolistiques manquent de réactivité. On aurait toutefois tort de réduire la dimension de la libéralisation à la politique européenne. L'Europe va, il est vrai, devenir progressivement le facteur d'organisation et de définition du système électrique au plan européen. Mais il se produit dans le même temps toute une série de facteurs de mutation qui convergent, à la fin des années 80 et au début des années 90 , vers ce processus de libéralisation. 
Les mutations sont tout d'abord technologiques. Dans le domaine électrique, les rendements croissants enregistrés durant un siècle faisaient dépendre directement le prix de revient unitaire du Kwh de la taille de l'équipement de production, poussant de manière imparable à la concentration et à la construction de grosses centrales nucléaires. Or aujourd'hui, la construction de micro-centrales de production utilisant le gaz naturel, en particulier avec co-génération, s'avère pratiquement aussi rentable et engendre une rupture avec un siècle d'évolution technico-économique du secteur. Dans le même temps, l'internationalisation contraint chacun des acteurs électriques, acteurs nationaux pour l'essentiel jusqu'aux années 80 , à adopter des stratégies internationales et à se concevoir comme des acteurs internationaux. L'électricité, comme tous les autres secteurs du service public, s'est internationalisée à vitesse rapide, et il ne s'agit pas là simplement d'intégration européenne. Autre facteur de mutation : la diversification des demandes et des besoins, une meilleure prise en considération des problèmes de qualité, l'individualisation du service en fonction du besoin de chacun sont autant de facteurs clé d'évolution. Le client exige aujourd'hui des solutions sur mesure, en opposition au principe traditionnel d'égalité. Par ailleurs, les dysfonctionnements des modes antérieurs de régulation et d'organisation ont mis en lumière, dans beaucoup de pays européens dont les services publics étaient protégés par des situations de monopole, une certaine inefficacité et des besoins avérés de modernisation, entraînant en retour une demande de modification du mode de production. Se greffent sur cette situation les stratégies d'acteurs et de groupes industriels et financiers qui jouent un rôle majeur dans ce processus d'évolution, en cherchant à concurrencer sur les segments d'activité les plus rentables les anciens monopoles nationaux ou territoriaux. Enfin, la force des thèses néo-libérales dans les années 80-90 vient conforter le discours sur les vertus de la concurrence.

Tout cela converge pour expliquer la libéralisation telle qu'on va l'enregistrer secteur par secteur, orchestrée, intégrée et organisée au niveau de l'Union européenne mais dont on aurait tort de ne voir qu'une cause européenne. Elle va commencer par les secteurs de l'énergie, des transports et des communications, pour ensuite s'étendre à d'autres activités et domaines (santé, éducation).

Objet d'un long cheminement et de compromis, l'ouverture à la concurrence au niveau européen va néanmoins être le révélateur d'une série de profondes transformations. Dans des secteurs ayant jusqu'alors connu un monopole national, l'opérateur historique ne peut évidemment que perdre des parts de marché et sera conduit, en tant qu'acteur économique cherchant à assurer sa survie et son développement, à diversifier ses activités, développer ses exportations (EDF va devenir le premier exportateur d'électricité au plan européen), mais aussi à s'implanter dans les pays voisins, pour au moins compenser ses pertes de marché à l'intérieur par des gains de parts de marché à l'extérieur. Ce qui nous situe dans une stratégie de fusions-acquisitions au fur et à mesure que se présentent des potentialités en Europe.

Mais dans le même temps, l'introduction de la concurrence génère un changement profond de culture interne (mode d'organisation et de management, culture commerciale et managériale). L'Etat perd également sa légitimité à être l'actionnaire et le dirigeant unique d'une entreprise qui devient un groupe européen, sinon mondial, puisqu'il n'a aucune légitimité à définir la stratégie de développement de l'électricité en dehors du territoire national. Cela amène dès lors des demandes de réciprocité et EDF va progressivement se transformer en un groupe européen et mondial définissant sa stra- 
tégie au plan mondial, cycle de mutation qui, logiquement, débouche sur un changement de statut (transformation de l'établissement public à caractère industriel et commercial en société anonyme), et à terme également sur un processus de privatisation. Il est à noter toutefois que la loi actuelle indique que l'Etat conservera $70 \%$ du capital (pour mémoire, ce fut également le cas il y a quelques années pour France Télécom).

\section{Un constat de déstabilisation}

Les principales caractéristiques du «modèle EDF » sont directement interpellées par l'évolution du cadre économique :

- le niveau national en tant que cadre de référence : on constate une rupture dans l'élaboration de la stratégie, le cadre de référence devenant l'Europe et le monde ;

- le paradigme du monopole : la concurrence devient le cadre de définition de l'organisation ;

- l'entreprise publique : elle fait place au paradigme de l'entreprise privée ;

- le service public: sans disparaître, il cède néanmoins progressivement la place à des logiques commerciales ;

- un modèle centralisé : il va tendre à un modèle plus décentralisé ;

- un système universel : c'est un système de plus en plus territorialisé qui se met en place avec l'introduction de phénomènes de différenciation territoriale ;

- un modèle intégré : il va devenir un modèle dé-intégré, traitant à part production, transport et distribution (séparation juridique, comptable, etc.) ;

- le jeu à trois acteurs : il va donner place à un jeu multi-acteurs ;

- un modèle stable durant 50 ans : ébranlé, il évolue aujourd'hui dans une période de changement et d'instabilité dont on ne connaît ni la durée ni l'issue et dont on ne peut savoir aujourd'hui si elle va permettre la reconstitution d'un modèle stable.

Dès lors, quels scénarios retenir pour l'avenir? On définira trois grandes orientations potentielles :

- l'entreprise EDF peut avoir comme avenir celui d'une entreprise nationale, européenne ou d'un groupe mondial : ceci représente dès lors trois possibilités pour l'avenir d'EDF (la première hypothèse semblant aujourd'hui toutefois la moins probable).

- l'activité d'EDF peut rester l'électricité (son pôle traditionnel), l'énergie (sous toutes ses formes) ou devenir celle d'un groupe multi-services à la manière de Veolia ou de Suez : là encore, trois avenirs possibles sont envisageables.

- les valeurs de l'entreprise peuvent rester celles du service public, céder le pas à celles d'un groupe multinational traditionnel ou bien évoluer vers des valeurs hybrides d'une 'entreprise de troisième type' (expression employée par le précédent président d'EDF) conjuguant les objectifs de service public avec la réalité de l'existence d'un groupe.

Arithmétiquement, si l'on retient ces trois grandes options et que l'on décline leurs diverses sous-variantes, on est donc confronté, selon notre analyse, à 27 scénarios possibles, pas tous équi-probables, mais qui suffisent à considérer comme très ouvert l'avenir d'EDF.

\section{La gestion déléguée}

Le deuxième "modèle français » est celui, très ancien, de gestion déléguée des services remontant à l'Ancien Régime. L'autorité publique qui, en effet, définit les objectifs et les missions de service public, peut soit assurer elle-même un service en direct - service 
municipal, régie, etc. - soit le déléguer à une entreprise : entreprise privée, société à capitaux mixtes, association, etc.

L'autorité publique se transforme dès lors en autorité organisatrice (concept employé dans le domaine des transports). Dans ce processus de délégation, elle définit, de manière théorique, les objectifs, missions et finalités (cahier des charges) puis lance un appel d'offres européen qui met en concurrence l'ensemble des acteurs susceptibles d'y répondre. En échange, elle peut accorder un monopole territorial et temporel pour une durée de temps à déterminer : elle peut se donner les moyens de fixation et de contrôle des tarifs et des investissements, et peut enfin assurer un rôle de contrôle et de régulation.

En France, certains secteurs pratiquent de longue date la gestion déléguée. C'est le cas, dès le XIXe siècle, de l'eau et de l'assainissement. En effet, les communes françaises n'ont, à la fin du XIXe siècle, pas le droit d'exercer une activité économique, sauf en cas de défaillance avérée du marché. Dans le domaine de l'eau et de l'assainissement, les communes auront dès lors la responsabilité d'avoir des fontaines publiques, mais n'auront pas le droit d'établir des réseaux de distribution d'eau, qui seront délégués à des entreprises privées. Cette situation va certes évoluer, mais les régies municipales telles qu'on les connaîtra par la suite seront des municipalisations intervenant dans le domaine de l'eau et de l'assainissement dans l'entre-deux-guerres, assouplissement accordé à l'interdiction d'activité économique des communes. Dès lors, cette tradition très ancienne de la gestion déléguée continue d'exister aujourd'hui en France.

Dans le même temps, on observe dans ce secteur de l'eau et de l'assainissement (mais aussi des transports urbains) des phénomènes de technicisation croissante et la multiplication de normes de qualité extrêmement contraignantes qui vont amener des besoins nouveaux en termes d'expertise technico-économique (traitement des eaux, des déchets, etc.). Or, le contexte d'émiettement communal français (36000 communes, 22000 autorités organisatrices décidant de gérer ou de déléguer les services de l'eau et de l'assainissement) pose ici un problème fondamental face à la concentration des besoins qui dans le même temps, va contribuer à générer une augmentation des coûts dans ces deux secteurs. En effet, du fait des normes de qualité et environnementales, les coûts augmentent en dépit des progrès de productivité et des progrès technologiques. On est face aussi à des besoins d'efficacité, pas toujours à la portée des régies (là encore, pas à l'abri de situations de monopole local). L'ensemble de ces constats convergent avec une particularité française des années 60 et 70, à savoir les besoins de financement de la vie politique française : il n'existe pas de financement public des partis jusqu'aux années 90 et la délégation des missions de service public à des compagnies privées sera alors un des moyens utilisés pour financer la vie politique française (tous partis confondus).

L'ensemble de ces facteurs se conjugue pour expliquer le développement rapide de la délégation de la gestion de l'eau, puis de l'assainissement, à des entreprises privées qui progressivement vont se concentrer, se développer, et se constituer en un oligopole de trois grands groupes de services. De la même manière qu'il existait un oligopole de l'électricité dans l'entre-deux-guerres, se constitue dans la période plus récente un oligopole dans les domaines de l'eau et de l'assainissement. Mais les groupes vont s'intégrer verticalement et horizontalement et proposer aux élus locaux un ensemble de prestations nécessaires pour les services publics locaux (transports urbains, gestion des parkings, des cantines, etc.). Trois grands groupes multi-services se sont ainsi constitués - Veolia (ex-Vivendi, ex-Générale des Eaux), Suez-Lyonnaise des Eaux et la Saur, de 
moindre importance - et s'internationalisent. Au fur et à mesure que des villes dans le monde voudront moderniser leurs secteurs de l'eau et de l'assainissement et feront appel à des concessions, ils pourront alors proposer leurs services et la France finit par exporter son 'modèle de gestion déléguée'. Internationalisation très rapide et diversification font que ces groupes deviennent des leaders mondiaux pour l'eau, l'assainissement et les services.

Or, ce deuxième modèle n'est pas non plus sans poser problème. Le profond déséquilibre structurel d'informations et de compétences qui existe entre les trois grands groupes et les 36000 communes ou 22000 autorités organisatrices autorise là encore à parler de phénomène de 'capture du régulateur' par l'opérateur, sans doute avec plus de force que dans le premier modèle. Bien que le 'régulateur' (autorité publique concédante) dispose depuis ces dernières années de quelques moyens supplémentaires, on constate une réelle difficulté de régulation publique de ces trois groupes. La concurrence oligopolistique est donc très relative, malgré bien sûr des procédures d'appels d'offre à échéance régulière de remise en concurrence : en effet, dans près de $90 \%$ des cas, le même groupe obtient la reconduction de son contrat, puisqu'il existe un consensus entre les trois grands groupes pour ne pas se livrer entre eux à une guerre effrénée. De fait, la concurrence porte davantage sur les marchés internationaux que sur le marché français. En conséquence, des rentes de monopoles se développent et les usagers sont particulièrement marginalisés, en particulier dans le domaine de l'eau puisque l'habitat collectif en France a cette particularité de ne pas avoir de compteur d'eau individuel (l'eau fait partie des charges locatives, sans lien direct avec la consommation). Cette absence de transparence est donc propice à générer de fortes suspicions sur les augmentations de prix et à mobiliser les populations.

\section{Perspectives de développement de la gestion déléguée de services publics}

Le développement de l'internationalisation des groupes entraîne dans le même temps des problèmes de profitabilité. En particulier les contrats pris par ces groupes dans certains pays en développement posent un réel problème de rentabilité. La diversification constatée des activités n'exclut néanmoins pas une certaine tendance à des reconcentrations, Suez axant sa stratégie sur l'eau et l'énergie, tandis que Veolia, face aux affres de Vivendi, tente à son tour de se focaliser sur certains cœurs de métier.

Par ailleurs, on remarque une volonté de se concentrer sur la gestion des contrats de délégation bien davantage que sur les investissements lourds, à longue durée de retour sur investissement, et pas toujours aussi rentables, en particulier sur certains marchés étrangers. La gestion de contrats de long terme est donc privilégiée aux investissements, alors même que la littérature actuelle sur les PPP (Public-Private Partnerships) insiste sur la solution consistant à utiliser les capitaux privés pour répondre aux besoins d'investissements lourds des collectivités publiques. On voit là que cette démarche se heurte aux intérêts des groupes tels qu'ils sont aujourd'hui constitués. Ces groupes ne demandent aujourd'hui pas tant au plan européen des libéralisations en tant qu'ouverture à la concurrence (ils sont plutôt satisfaits d'avoir des monopoles locaux, territoriaux et temporels), mais se concentrent sur une demande de généralisation d'appels d'offres, et donc sur la mise en place d'une concurrence oligopolistique. Il existe donc aujourd'hui tout un débat au sein des institutions européennes et de la Commission: l'avenir des services publics passe-t-il par la systématisation des appels d'offres ? La gestion "in house », c'est-à-dire à l'intérieur de la collectivité en régie 
municipale ou par un service municipal, a-t-elle un avenir ou faut-il, s'il y a obligation de service public, avoir obligation d'appels d'offres ? Le débat est aujourd'hui en cours.

La dernière question que souhaite soulever cette contribution est la suivante : va-t-on vers des groupes transsectoriels, comme cela a été l'ambition affichée par Jean-Marie Messier quand il était PDG de Vivendi, avec une place hégémonique dans la structuration des sociétés? C'est un des avenirs possibles, il y en a d'autres, mais nous ne décrirons pas là les vingt-sept scenarii possibles.

\section{Indications bibliographiques}

BAUBY P., Les services publics, Paris, Flammarion, 1997

Bergougnoux J., Services publics en réseau: perspectives de concurrence et nouvelles régulations. Commissariat général du Plan, Paris : La Documentation Française 2000

Cohen É., Henry C., Service public, Secteur public, Rapport au conseil d'analyse économique, Paris : La Documentation française, 1997

Henry C., Matheu M., Jeunemaitre A., (éd.): Réguler les services publics en réseaux: l'expérience européenne. Commissariat général du Plan, Paris : La Documentation Française, 2003 


\title{
Le secteur de l'eau en Allemagne - entre libéralisation et modernisation
}

\author{
Rina BOHLE ZELLER
}

Alors que la France et l'Angleterre ont depuis longtemps ouvert leurs marchés de l'eau au secteur privé, la fourniture des services d'approvisionnement en eau potable et l'assainissement des eaux usées reste, en Allemagne, sous la responsabilité des communes. Le secteur de l'eau s'y caractérise ainsi par une multitude de structures de taille variable nées selon les besoins locaux. Le débat sur la libéralisation de ces services, qui avait pris de la vigueur avec la libéralisation du secteur de l'énergie en 1998, a connu un tournant en 2002 quand le Bundestag s'est prononcé contre une libéralisation tout en reconnaissant la nécessité d'une modernisation structurelle du secteur. La stratégie à laquelle semblent adhérer aujourd'hui tous les acteurs concernés vise à améliorer l'efficacité et la compétitivité des services d'approvisionnement et d'assainissement tout en laissant aux communes la responsabilité organisationnelle. Elle s'inscrit dès lors dans une logique générale de modernisation des services publics.

\section{Souveraineté administrative des communes}

En Allemagne, l'organisation des services d'approvisionnement en eau potable ainsi que d'assainissement des eaux usées relève des communes. Dans le jeu de la répartition des compétences qui caractérise la République fédérale, ce sont elles en effet qui disposent de la souveraineté administrative concernant l'organisation sur leur territoire des activités de "prévoyance » (Daseinsvorsorge), c'est-à-dire des services d'intérêt général. L'art. 28, § 2,1 de la Loi fondamentale stipule en effet: "Aux communes doit être garanti le droit de régler, sous leur propre responsabilité, toutes les affaires de la communauté locale, dans le cadre des lois ». Conformément au principe de subsidiarité et à la séparation entre pouvoirs législatif et administratif qu'il implique, les compétences administratives en la matière échappent donc aux Bund et aux Länder. Ces derniers disposent en revanche de compétences législatives étendues : ils mettent en œuvre sur leur territoire (donc transcrivent en droit régional) la législation-cadre du Bund sur l'eau (Wasserhaushaltsgesetz). Cette compétence se double d'une autre, qui est, elle, exclusive : la définition du droit communal et, plus précisément, la répartition des compétences entre l'échelon du Land et celui des communes. Le Bund est par ailleurs en charge du droit de la concurrence, de l'adaptation des lois allemandes à la réglementation européenne ou encore de la protection durable des ressources naturelles. De ce seul fait, le marché allemand de l'eau se caractérise par une grande diversité des acteurs institutionnels et une grande variété des structures.

Les entreprises municipales au cœur de la Daseinsvorsorge

La souveraineté administrative des communes inclut le choix de la forme que prend la fourniture des services relevant de la Daseinsvorsorge. La commune peut ainsi détenir 
en propre les structures d'approvisionnement et d'assainissement de l'eau, déléguer la gestion de ces services au secteur privé ou les assurer via des entreprises d'économie mixte. Cette latitude de décision, quoique soumise au cadre légal défini par les Länder et au niveau fédéral, a contribué au développement d'une multitude de structures organisationnelles relevant soit du droit public soit du droit privé. Indépendamment du statut juridique, on constate souvent un regroupement des activités de la Daseinsvorsorge dans une seule entreprise de services municipaux, appelée Stadtwerk. En dehors des économies de coûts de fonctionnement, notamment en raison d'une gestion centralisée, cette organisation transversale permet à ces entreprises de compenser les pertes engendrées par un secteur d'activité (souvent le transport collectif) grâce aux bénéfices réalisés dans d'autres secteurs (en particulier l'électricité). Ce subventionnement croisé est critiqué pour mener à une sur-tarification dans les activités bénéficiaires et pour provoquer des distorsions de concurrence. Mais depuis la libéralisation du secteur de l'énergie, beaucoup d'entreprises municipales se sont séparées des services d'électricité et de gaz, ce qui réduit le nombre de possibilités de subventionner des activités déficitaires. S'y ajoute l'introduction d'une comptabilité d'engagement distincte par service.

\section{Daseinsvorsorge}

Le terme allemand de Daseinsvorsorge désigne des activités relevant des missions de service public, ou selon la nomenclature européenne, des «services d'intérêt économique général » dont le livre vert de la Commission européenne donne la définition suivante: «services de nature économique que les Etats membres ou la Communauté soumettent à des obligations spécifiques de service public en vertu d'un critère d'intérêt général ». Ces services sont souvent soumis aux obligations de service universel qui visent à garantir à tous les citoyens un accès abordable et une qualité de service définie. Il s'agit principalement de l'ensemble des services de réseaux (postaux, transports publics, énergie, etc.). La connotation du concept de Daseinsvorsorge est aussi sensible en Allemagne que celle de la notion de «service public » en France, bien que ne s'y mêlent généralement pas missions d'intérêt général et statut public des entreprises sauf dans le secteur bancaire (banques publiques) et celui de l'eau...

\section{Une multitude d'entreprises pour les services aux particuliers}

Contrairement à la France ou à l'Angleterre qui connaissent une forte concentration, le marché allemand de l'eau reste caractérisé par une multitude d'entreprises. Environ 6560 entreprises approvisionnent 81,6 millions d'habitants (Destatis, 2001), ce qui représente une moyenne de plus de 80 entreprises pour un million d'habitants; en France et en Angleterre, le même nombre de personnes est desservi par une seule entreprise. Mais cette moyenne masque une grande hétérogénéité : alors que 100 à 200 entreprises approvisionnent 100000 habitants dans les Länder à faible densité de population, une seule entreprise peut desservir plus de 100000 habitants (voire plus d'un million) dans les agglomérations urbaines. Cela fait qu'environ 100 entreprises fournissent la moitié de l'eau potable en Allemagne. Dans le secteur de l'assainissement, la structure est globalement semblable. Après la réunification, les 16 entités étatiques d'approvisionnement et d'assainissement de l'ex-RDA ont été transformées en 550 entreprises d'approvisionnement et 1050 structures d'assainissement. Les services 
de l'eau s'adressent pour l'essentiel aux particuliers : l'industrie est autonome à hauteur de $96 \%$ de sa consommation (pour $70 \%$ en Angleterre, et $81 \%$ en France). Mais les sociétés d'assainissement interviennent à hauteur de $40 \%$ environ dans le traitement des eaux usées industrielles.

Tableau 1 : Part de marché des différentes entreprises du secteur de l'eau (2003)

\begin{tabular}{|c|c|c|}
\hline Entreprises relevant du droit public & Approvisionnement & Assainissement \\
\hline $\begin{array}{l}\text { entreprise à caractère public gérée selon les principes } \\
\text { de gestion publique (Regiebetrieb) }\end{array}$ & $0,4 \%$ & $19,7 \%$ \\
\hline $\begin{array}{l}\text { entreprise à caractère public gérée selon les principes } \\
\text { de gestion commerciale (Eigenbetrieb) }\end{array}$ & $14,9 \%$ & $42,7 \%$ \\
\hline $\begin{array}{l}\text { établissement de droit public } \\
\text { (Anstalt des öffentlichen Rechts) }\end{array}$ & - & $17,0 \%$ \\
\hline $\begin{array}{l}\text { association des eaux et syndicat intercommunal } \\
\text { (Wasser- und Zweckverband) }\end{array}$ & $22,2 \%$ & $12,8 \%$ \\
\hline Entreprises relevant du droit privé (S.A./S.A.R.L.) & Approvisionnement & Assainissement \\
\hline $\begin{array}{l}\text { entreprise publique relevant du droit privé } \\
\text { (öffentliche Gesellschaft / Eigengesellschaft) }\end{array}$ & $30,2 \%$ & - \\
\hline $\begin{array}{l}\text { entreprise d'économie mixte } \\
\text { (öffentlich-private Beteiligungsgesellschaft) }\end{array}$ & $28,8 \%$ & - \\
\hline $\begin{array}{l}\text { entreprise privée } \\
\text { (le plus souvent de statut SA ou SARL) }\end{array}$ & $3,5 \%$ & - \\
\hline autres & - & $7,8 \%$ \\
\hline
\end{tabular}

Source : Branchenbild der deutschen Wasserwirtschaft, 2005. NB : l'échantillon analysé couvre, avec 1266 entreprises, $76 \%$ de l'approvisionnement en eau potable et, avec 906 entreprises, $52 \%$ des services d'assainissement; les entreprises hors échantillon sont notamment des petites entreprises à caractère public (Regiebetrieb, Eigenbetrieb).

\section{Approvisionnement : droit privé ; assainissement : droit public}

Le statut des entreprises révèle un partage du marché par segments. Dans celui de l'approvisionnement, on observe une nette tendance à la privatisation: la part des entreprises de droit privé est passée de $17 \%$ en 1986 à $62,5 \%$ en 2003 . A l'inverse, dans celui de l'assainissement, les entreprises de droit public sont majoritaires (plus de $90 \%$ en 2003).

\section{Approvisionnement : ouvert au secteur privé dès les années 1990}

La tendance à la concentration (coopération des communes dans le cadre des syndicats intercommunaux) et à la participation du secteur privé, notamment dans l'approvisionnement, prend ses origines dans les années 1990 avec la hausse des besoins d'investissement dans les nouveaux Länder parallèlement à des exigences plus élevées dans le domaine de la protection de l'environnement et des standards de qualité. Leur situation budgétaire tendue incitait alors les communes à s'orienter vers des stratégies alternatives. Par ailleurs, les exigences croissantes de transparence ont fait que les entreprises communales sans personnalité morale propre (Regiebetrieb), qui ne sont pas obligées de tenir une comptabilité d'engagement (qui facilite le contrôle des coûts), ont connu une forte baisse de leur nombre dans le secteur de l'assainissement (de 44,0 \% en 
1997 à 19,7 \% en 2003) et ne représentent plus aujourd'hui qu'une part de marché de $0,4 \%$ dans le secteur de l'approvisionnement.

\section{L'assainissement reste largement une activité régalienne}

La participation accrue d'acteurs privés dans le secteur de l'approvisionnement limite le nombre d'entreprises communales du type Eigenbetrieb (passé de 63,3\% en 1986 à $14,9 \%$ en 2003). Mais cette forme d'entreprise a connu une hausse dans l'assainissement (de 30,0\% en 1997 à 42,7 \% en 2003), où le secteur privé ne reste que faiblement représenté. La tendance inversée s'explique principalement par la considération différenciée des services de l'eau. Alors que l'approvisionnement est depuis toujours ouvert au privé, l'assainissement était traditionnellement considéré comme une activité régalienne. En effet, il a fallut attendre la modification de la loi Wasserhaushaltsgesetz en 1996 pour autoriser la participation du secteur privé dans les entreprises d'assainissement. Celles-ci sont dès lors encore majoritairement publiques, mais avec une tendance à des formes organisationnelles permettant une amélioration de la transparence des coûts. Avec des besoins d'investissement annuels d'environ 5,5 milliards $€$ dans l'assainissement, le secteur privé est cependant un important soustraitant : il intervient pour $70 \%$ environ de ces investissements.

\section{Deux types de contrôle des prix}

Malgré ces évolutions, les communes détiennent généralement la majorité du capital $(>51 \%)$ de ces sociétés. Contrairement à la France et à l'Angleterre, les communes ont alors de forts pouvoirs de contrôle et de surveillance, ce qui s'explique par la nécessité d'une influence publique pour assurer la protection durable des ressources, la qualité du service ou encore un contrôle du niveau des prix. A ce titre, il est important de souligner qu'il n'existe pas de réglementation de prix des services de l'eau comme dans le secteur des télécommunications ou de l'électricité (Gabel, 2006). La différence d'approche juridique des deux segments se reflète au niveau du contrôle des prix. En effet, pour les services d'assainissement (droit public), le prix est calculé par l'entreprise selon le principe de couverture des dépenses, et décidé et appliqué en tant que redevance par la commune. En cas de plainte, son montant et sa composition sont analysés par des tribunaux ou des commissions de contrôle administratif. A l'inverse, le prix des services d'approvisionnement (droit privé) correspond le plus souvent à la rétribution d'une prestation. Et le contrôle des prix, y compris en cas de doutes par rapport à sa justification, relève dès lors généralement de l'office des cartels du Land. Ce type de régulation ex post est justifié par le fait que la plupart des entreprises publient les prix pratiqués et permettent ainsi aux consommateurs d'exercer un contrôle par comparaison.

\section{Années 1990 : débats sur la libéralisation de l'eau et la gestion communale}

Alors que les acteurs du marché de l'eau n'entrent que peu en concurrence pour la répartition des ressources naturelles (seulement 20,9\% des eaux annuellement renouvelées sont partagées par tous les acteurs), la gestion communale des services de l'eau connaît de vives critiques notamment depuis les années 1990. Le besoin d'investissement accru, essentiellement pour la remise à niveau des réseaux des Länder de l'est, ainsi que pour l'entretien des installations et leur adaptation à des standards environnementaux de plus en plus exigeants, avait en effet provoqué d'importantes hausses de 
prix (+43\% dans les années 1990). Parallèlement, les effets conjugués de l'exode urbain qu'ont connu certaines villes est-allemandes et de la baisse de la consommation (passée de 1471 par personne et par jour en 1990 à un niveau stable d'environ 1301 depuis 1995) commençaient à peser sur les budgets communaux. La nécessité de procéder à des ajustements structurels et de veiller à l'hygiène des parties sous-utilisées des réseaux (évacuation des eaux stagnantes) contribuaient alors à la hausse des tarifs (répartition des coûts fixes sur les consommateurs). Face à cette situation, et face à la situation budgétaire tendue de beaucoup de communes allemandes, de nombreuses voix ont exigé alors de nouveaux modes de gestion, ouvrant ainsi le débat concernant la libéralisation du secteur de l'eau. Un débat où fut soulignée la faible performance des entreprises allemandes sur le marché international de l'eau en raison de leur petite taille, contrairement aux grands groupes français, par exemple.

\section{Appliquer le droit de la concurrence à l'eau ?}

La libéralisation du marché de l'énergie en 1998 et la révision afférente de la Loi contre les entraves à la concurrence (Gesetz gegen Wettbewerbsbeschränkungen, GWB, alors dans sa version de 1990), ont l'effet d'un catalyseur. Au cœur de la discussion figure la question du maintien du $\S 103$ pour le secteur de l'eau (il a été abrogé pour le secteur de l'énergie lors de la révision de la Loi GWB en 1998). Cet article, qui excluait du droit commun de la concurrence les activités des entreprises de l'eau, du gaz et de l'électricité, autorisait en effet les communes à maintenir un statut de monopole local pour ces services en leur permettant de conclure des accords de coopération intercommunale et d'exclure des opérateurs tiers de l'utilisation de leurs infrastructures (évitant ainsi la compétition de plusieurs opérateurs sur le même marché). Or dans le secteur de l'eau, la suppression du $\S 103$ n'aurait eu qu'un impact limité dans la mesure où la Loi GWB ne s'applique qu'aux entreprises relevant du droit privé. Parallèlement, le maintien des privilèges fiscaux dont bénéficient les entreprises de droit public (exonérées de l'impôt sur les sociétés et de la TVA), notamment dans le domaine de l'assainissement, était lui aussi critiqué. Les distorsions de concurrence et l'établissement de barrières à l'entrée pour le secteur privé constituaient le principal argument pour l'abolition du traitement fiscal privilégié.

\section{Vers une mise en concurrence pour créer le marché}

Une étude sur la libéralisation du marché de l'eau commanditée par le ministère fédéral de l'Economie en 2001 permet de conclure à l'existence de nombreux avantages liés à une libéralisation progressive sous la condition d'un ajustement du cadre réglementaire, notamment dans le domaine de la régulation des prix. Selon cette étude, la solution consistait notamment dans la promotion des procédures permettant une compétition pour le marché (Wettbewerb um den Markt), par exemple par le biais des concessions attribuées à un opérateur privé sur appel d'offre pour une période limitée. En effet, une libéralisation visant la concurrence dans le marché (la coexistence de plusieurs opérateurs utilisant la même infrastructure ou Wettbewerb im Markt semblait (et semble toujours) peu réaliste compte tenu des conditions techniques. La petite taille de la plupart des réseaux, et donc le faible nombre de clients, rend la coexistence de plusieurs opérateurs peu efficace en raison de l'importance des coûts fixes à répartir entre les utilisateurs. L'interconnexion de plusieurs réseaux pour assurer un agrandissement des 
marchés d'approvisionnement et d'assainissement nécessite des investissements importants. De plus, l'injection de l'eau provenant d'origines différentes dans le même réseau peut avoir pour conséquence la baisse de la qualité de l'eau potable (en raison des processus chimiques des agents contenus dans l'eau ou des temps de transports importants). La concurrence dans le secteur de l'eau correspond alors le plus souvent à une compétition des acteurs publics et privés pour obtenir des parts dans une entreprise bénéficiant du statut d'un monopole local.

\section{Front commun contre la libéralisation...}

Durant la même période, les communes, les fédérations d'entreprises communales (Verband kommunaler Unternehmen, VKU ; Bundesverband der deutschen Gas- und Wasserwirtschaft, BGW...), le syndicat ver.di (Bourgeois, 2006), ainsi que les consommateurs, s'opposaient vivement à la libéralisation. Leur refus reposait essentiellement sur l'impact qu'aurait une perte d'influence des communes dans les domaines de l'assainissement et de l'approvisionnement. Elle était supposée se traduire par une réduction des effectifs (environ 100000 personnes sont actuellement employées dans le secteur de l'eau), par une hausse des prix, une baisse de la qualité des services et un impact négatif sur l'environnement et la gestion durable des ressources naturelles. La critique exprimée se dressait essentiellement contre les privatisations matérielles (vente de l'entreprise) et formelles (délégation de la gestion et du maintien du réseau à un opérateur privé pour une longue durée via une concession), arguant que ce type de 'concurrence' remplacerait un monopole public par un monopole privé, plus difficilement contrôlable. La faible augmentation du nombre d'entreprises privées dans le secteur de l'approvisionnement (de 1\% en 1986 contre 3,5\% en 2003) permet d'illustrer cette position.

\section{... de l'assainissement plus que de l'approvisionnement}

L'opposition à la libéralisation, à laquelle se sont ralliés des parlementaires de la majorité d'alors (SPD/Verts) tout comme certains chrétiens démocrates, était néanmoins consciente de la nécessité de trouver des solutions permettant la gestion durable des services de l'eau. Face à des budgets communaux tendus, cette opposition se révélait néanmoins modérée, notamment dans le secteur de l'approvisionnement. La forte hausse de la part des entreprises d'approvisionnement en économie mixte (elle passe de $3,3 \%$ en 1986 à $28,8 \%$ en 2003) y contribue. Dans le souci de la protection durable de l'environnement, l'opposition à la libéralisation se cristallisera d'autant plus aisément sur le segment de l'assainissement qu'il relève des prérogatives régaliennes des communes.

\section{2 : stratégie de modernisation plutôt que libéralisation}

Le débat connaît un tournant en 2002 quand le Bundestag se prononce majoritairement contre une libéralisation du secteur de l'eau. Le $§ 103$ de la Loi GWB est maintenu, et la nouvelle version de la loi, adoptée en 2005, prorogera ce régime d'exception au droit commun de la concurrence. Mais dans le même temps, le Bundestag constate la nécessité d'une modernisation du secteur. La «stratégie de modernisation » adoptée alors vise notamment à améliorer l'efficience et la compétitivité des entreprises d'approvisionnement et d'assainissement. Elle se trouve confirmée par la résolution du Parlement européen de janvier 2004 s'exprimant contre la libéralisation du secteur de l'eau. 


\section{Les mesures clefs de la " stratégie de modernisation »}

- Benchmarking des entreprises ;

- promotion des coopérations intercommunales ;

- promotion des partenariats public-privé ;

- harmonisation des régimes fiscaux de l'approvisionnement et de l'assainissement ;

- harmonisation du droit communal des Länder (Gemeindeordnungen) ;

- promotion de la participation des entreprises allemandes au marché de l'eau international

\section{Principaux handicaps : la disparité des réglementations...}

Le besoin d'une modernisation semble être accepté par tous les acteurs, qui s'accordent sur l'importance d'une amélioration de l'efficience des entreprises concernées. Tous les points soulevés par la stratégie de modernisation ne connaissent cependant pas la même attention des acteurs et des médias, qui se concentrent notamment sur l'harmonisation du système fiscal (toujours à l'étude) et le benchmarking qui devrait permettre de comparer l'efficience des entreprises. En revanche, la promotion des coopérations intercommunales, ainsi que les partenariats public-privé soulèvent d'autres questions. La promotion des coopérations intercommunales vise à accroître la taille des réseaux afin de réaliser des économies d'échelle (et de créer des structures suffisamment grandes pour pénétrer le marché international). Mais cette stratégie nécessite l'assouplissement et, plus encore, l'harmonisation d'un droit communal qui varie très largement d'un Land à l'autre, afin de permettre aux entreprises communales d'exercer des activités au-delà de leur territoire. Les Länder ont certes engagé des réformes dans ce sens, mais il s'agit d'un chantier d'une haute complexité et dont les enjeux ne concernent au fond la problématique du marché de l'eau qu'à la marge. Un recours à plus de partenariats public-privé, qui dépassent la simple participation du secteur privé dans une entreprise communale (par exemple les contrats de concessions) nécessite une adaptation du cadre réglementaire actuel, notamment par rapport aux appels d'offre et au niveau de la régulation tarifaire. Bien que toutes les entreprises soient tenues de respecter les critères européens concernant les appels d'offre (transcrites en droit national lors de la réforme de la Loi GWB en 2005), il n'existe pas encore de procédures harmonisées en Allemagne, notamment en ce qui concerne les modalités contractuelles pour les concessions (modèles de contrats, par exemple). La situation pourrait évoluer avec la réforme du fédéralisme et les débats sur les projets européens tels celui d'une Directive sur les services d'intérêt général ou sur la mise en place d'une réglementation européenne en matière d'appels d'offre dans le domaine communal.

\section{... et l'absence de standardisation des procédures de benchmarking}

L'introduction du benchmarking (malgré l'absence de concurrence directe) vise à inciter à des ajustements en cas de sous-performance. Il fonctionne sur la base d'une comparaison d'indicateurs relevant de domaines divers et qui comprennent, en application du système international IWA (International Water Association) : la sécurité de l'approvisionnement et de l'assainissement, la qualité, le service clientèle, la gestion durable ainsi que l'efficience économique. Ces indicateurs sont fournis par l'entreprise et regroupés par l'entité organisatrice, souvent les fédérations d'entreprises communales (VKU, BGW...). En cas de divergence des indicateurs avec la moyenne, l'entreprise doit en analyser les causes et procéder le cas échéant à des corrections. Actuellement, il n'existe pas d'obligation de participer à un benchmarking; les acteurs se prononcent 
d'ailleurs contre une telle obligation en exigeant la possibilité d'une participation volontaire. Les projets pilotes se développent, mais ils n'appliquent pas tous la même procédure et ne concernent souvent qu'un petit nombre de participants. Afin de permettre une comparaison à l'échelle de l'ensemble du pays, voire internationale, les procédures devront être standardisées.

AU VU Des évolutions aCtuelles, le marché allemand de l'eau restera fortement marqué par l'influence communale et par une hétérogénéité de solutions organisationnelles. Même si la stratégie de modernisation comprend la promotion de structures plus grandes, un changement ne devrait pas relever de l'immédiat, bien que certaines villes ou régions aient déjà avancé dans cette voie, comme en atteste la coopération communale dans l'approvisionnement en eau potable en Hesse (Hessenwasser GmbH \& Co. $K G)$ ou le partenariat public-privé à Dresde dans le domaine de l'assainissement (Stadtentwässerung Dresden GmbH). Dès lors, la vocation actuelle de l'Allemagne n'est vraisemblablement pas de devenir un grand pourvoyeur de services d'eau sur le marché international à l'instar de la France, surtout depuis l'annonce du groupe RWE de se séparer de Thames Water (Angleterre) et d'American Water (Etats-Unis).

\section{Indications bibliographiques :}

Arbeitsgemeinschaft der deutschen Trinkwassertalsperren e.V. (ATT), Bundesverband der deutschen Gasund Wasserwirtschaft et al., Branchenbild der deutschen Wasserwirtschaft 2005, Bonn, 2005

BouRGEOIS I., «Ver.di refuse les 40 heures pour construire sa légitimité », in : Regards sur l'Economie allemande, 2006, $\mathrm{n}^{\circ} 76$ (mai 2006)

Bundesministerium für Umwelt, Naturschutz und Reaktorsicherheit, Privatisierung in der Wasserwirtschaft, Berlin, 2002

Bundesministerium für Wirtschaft und Arbeit, Wasserleitfaden, Documentation n`547, Berlin, juillet 2005

Clausen H., Scheele U., «Strukturwandel in der Wasserversorgung-zwischen Liberalisierung und nachhaltiger Entwicklung », édité par Niedersächsisches Institut für Wirtschaftsforschung (NIW), in : Umwelt und Wirtschaft in Niedersachsen : Märkte, Innovationen, Chancen, Anreize und Instrumente, 2003

Ewers H.-J., Botzenhart K., SAlzWedel J., Kraemer R.A., «Optionen, Chancen und Rahmenbedingungen einer Marktöffnung für eine nachhaltige Wasserversorgung », étude commanditée par le Bundesministerium für Wirtschaft und Arbeit, Berlin, juillet 2001

GABEL M., « Régulation de l'énergie : l'exception allemande » in : Regards sur l'Economie allemande, 2006, $\mathrm{n}^{\circ} 76$ (mai 2006)

LuX A., ScheEle U., SChramm E., «Benchmarking in der Wasserwirtschaft-Möglichkeiten und Erweiterungen des Benchmarkings um soziale und ökologische Aspekte », networks working paper $\mathrm{n}^{\circ} 17$, Berlin, juin 2005 
- II -

EMPLOI PUBLIC ET GESTION DES RESSOURCES HUMAINES 



\title{
La fonction publique en Allemagne : ouverte à la réforme ou réfractaire au changement ?
}

\author{
Sabine KUHLMANN
}

« La force d'inertie de l'administration publique allemande est et a toujours été considérable. Elle est parfois effrayante. » (Ellwein 1997, p. 324)

La présente contribution traite des structures, des évolutions et des tentatives de réforme de la fonction publique en Allemagne, sachant que l'on prête traditionnellement à l'administration publique, (pour le lui reprocher), une force d'inertie qui limiterait sa capacité de réforme et rendrait difficile son changement institutionnel.

Les intérêts à « conserver » et à « maintenir » un certain statu quo sont réputés particulièrement forts dans la fonction publique. Cela tient au fait que ce secteur présente des problèmes de pilotage spécifiques, les objets de la réforme (administration/personnel administratif) en étant également les sujets, ceux qui conçoivent et appliquent les mesures. Cette identité sujet/objet accroît les risques de blocages dans le processus de réforme (Jann 2001, p. 330 sv. ; Benz 2004, p. 22). Les nombreux changements - parfois majeurs - traversés ces dernières années par la fonction publique de la République fédérale contredisent toutefois ce présupposé d'une force d'inertie "considérable » voire « effrayante ». L'analyse plus fine des mesures concrètes et l'ampleur effective des transformations au cours de la dernière décennie font apparaître une capacité de changement et une ouverture aux réformes tout à fait remarquable - quoique variable selon les échelons territoriaux et les branches de l'administration.

C'est dans ce contexte d'observations et d'hypothèses « concurrentes » concernant l'(in)aptitude à la réforme et au changement de l'administration et des services publics que nous analyserons les grandes évolutions de la fonction publique en Allemagne. Notre question centrale sera la suivante : les structures et modèles de fonctionnement traditionnels de la fonction publique allemande ont-ils effectivement été soumis à des ruptures et à des changements remettant en cause le système traditionnel de gestion des ressources humaines, et, si oui, dans quelle mesure ? Constate-t-on au contraire une certaine continuité dans le temps ? Comment expliquer ce phénomène ? Pour répondre à ces interrogations, il conviendra tout d'abord de rappeler les structures et caractéristiques fondamentales de la fonction publique dans le système fédéral allemand. Nous retracerons ensuite les principaux changements intervenus au cours des quinze dernières années pour en examiner les répercussions en termes de personnel. Nous reviendrons pour conclure sur la question centrale de cette contribution : celle de la continuité et du changement dans la fonction publique allemande. 


\section{La fonction publique dans le système fédéral allemand : structures et caractéristiques fondamentales}

\section{Répartition des compétences}

En Allemagne, la fonction publique se caractérise par deux principes distincts. D'une part, elle est marquée par un haut niveau de décentralisation et de variabilité, qui traduit la configuration constitutionnelle dans un État fédéral et le « fédéralisme administratif » à l'allemande. Ainsi à la différence très nette de la France (voir Kuhlmann, 2006) la majorité des fonctionnaires allemands sont employés à un échelon régional et local (Länder, municipalités), et les «employeurs » territoriaux disposent de larges compétences décisionnelles en matière de personnel. Le système de qualification et la formation des élites de la fonction publique sont aussi fortement décentralisés (voir section 2.4). D'autre part, la fonction publique est le reflet emblématique de l' " État fédéral unitaire » souvent invoqué : les conditions de service, les structures de carrières et les qualifications requises pour les personnels des différents niveaux font l'objet de règles relativement homogènes dans l'ensemble du pays. La Fédération (Bund) dispose conformément à l'art $75 \mathrm{I}$ de la Loi fondamentale (Grundgesetz - GG) d'une compétence législative cadre sur le statut des personnels (notamment, loi cadre de la fonction publique), à laquelle les Länder «satisfont» ensuite (de manière le plus souvent homogène) par leurs propres dispositions, portant généralement sur les personnels régionaux et municipaux (p. ex. réglementations en matière de carrière). Le Bund définit également de manière unitaire la rémunération et les pensions des fonctionnaires, en évoquant sa propre « compétence législative concurrente » (art 74a I GG). Assumant leur autonomie sur les questions de personnel (Personalhoheit), dérivée de l'autonomie de gestion garantie par la Loi fondamentale (art 28 II GG), les municipalités sont soumises à des conditions cadres définies par la législation de la fonction publique et du travail, dont la compétence incombe au Bund et aux Länder. Les décisions relatives à la gestion et à la politique des ressources humaines (recrutement, avancement, dans certains cas licenciement, contrôle hiérarchique) relèvent en revanche de la compétence des municipalités (Kuhlmann/Wollmann, 1999).

\section{Organisation verticale et sectorielle de la fonction publique}

La répartition des personnels de la fonction publique entre les échelons territoriaux Bund, Länder, municipalités - donne une image éloquente de l'importance de ces différents «employeurs publics » et de la structure verticale de la fonction publique allemande (Kuhlmann/Röber, 2006 ; Kuhlmann, 2006b). Il faut d'abord constater qu'en Allemagne - comme dans de nombreux pays de l'OCDE - le secteur public et donc l'emploi public ont connu une forte expansion depuis l'après-guerre, avec, pour l'« ancienne » République fédérale, un bond de 57 à 78 postes pour mille habitants entre 1960 et 1990. Au cours des années 1960, les parts respectives des différents niveaux se sont établies dans des ordres de grandeurs restés caractéristiques jusque dans les années 1990 : les municipalités employaient tout juste un tiers des agents de la fonction publique, les Länder environ la moitié, et le Bund un cinquième. Globalement, les effectifs relatifs du Bund reflètent le rôle dévolu à l'administration fédérale allemande, compétente pour la formulation de la politique, la législation, la régulation et la programmation (Jann, 2004, p. 100), tandis que la mise en œuvre des programmes politiques incombe presque en totalité aux Länder (sous leur propre responsabilité) puis, 
au sein de ceux-ci, principalement aux administrations des arrondissements (Kreise), villes et communes. Ainsi, l'activité de l'administration fédérale se limite essentiellement aux domaines suivants : défense (64\% des personnels du Bund à elle seule), finances (48 000 employés), police des frontières et police criminelle fédérale (42 500 personnes), administration des voies navigables et de la navigation (17 000 personnes) et Affaires étrangères (9 000 personnes) (Bogumil/Jann, 2005).

La comparaison des trois échelons territoriaux (voir tableau 1) fait également apparaître que c'est parmi les personnels employés par les Länder que l'augmentation est la plus forte entre 1960 et 1990 (60\%). Ce phénomène s'explique en premier lieu par les importantes réformes scolaires et universitaires (compétence des Länder), associées à une forte augmentation du nombre d'enseignants. L'éducation emploie aujourd'hui environ un tiers de l'ensemble des personnels de la fonction publique allemande, les effectifs ayant été multipliés par quatre depuis 1960 à la suite de l'expansion du système scolaire. Outre les domaines de l'éducation et de la recherche, qui regroupent environ $47 \%$ des personnels des Länder, les principaux domaines de compétences des Länder sont la sécurité publique, la police et la justice (catégorie « services généraux »; environ $20 \%$ des personnels des Länder) et l'administration fiscale et financière (environ $7 \%$ ).

Tableau 1 : Emploi public par échelon territorial avant et après la réunification (1960-2001)*

\begin{tabular}{|cccccccc|}
\hline Année & \multicolumn{2}{c}{ Bund } & \multicolumn{2}{c}{ Länder } & \multicolumn{2}{c|}{ Municipalités } & Total \\
& en milliers & en $\%$ & en milliers & en $\%$ & en milliers & en \% & en milliers \\
1960 & 361 & 18,5 & 950 & 48,6 & 642 & 32,9 & 1953 \\
1970 & 537 & 21,4 & 1210 & 48,3 & 758 & 30,3 & 2504 \\
1980 & 553 & 18,2 & 1568 & 51,5 & 920 & 30,2 & 3042 \\
1990 & 554 & 17,9 & 1536 & 49,7 & 1002 & 32,4 & 3092 \\
1993 & 579 & 14,3 & 2012 & 49,6 & 1469 & 36,2 & 4060 \\
1996 & 508 & 13,9 & 1909 & 52,3 & 1233 & 33,8 & 3649 \\
1999 & 479 & 14,5 & 1767 & 53,4 & 1062 & 32,1 & 3308 \\
2002 & 448 & 15,0 & 1583 & 53,1 & 951 & 31,9 & 2982 \\
évol. 1960/1990 en \% & 53,5 & & 61,7 & & 56,1 & & 58,3 \\
évol. 1993/2002 en \% & $-22,7$ & & $-21,3$ & & $-35,2$ & & $-26,6$ \\
\hline
\end{tabular}

Source : Kuhlmann/Röber 2006 (parmi d'autres références.). *Uniquement emplois à plein temps des collectivités territoriales (Bund, Länder, municipalités) ; hors chemins de fer fédéraux, syndicats de communes et « fonction publique indirecte ».

Les effectifs municipaux ont connu une croissance considérable, avec une augmentation de plus de moitié (56\%) entre 1960 et 1990, traduisant l'augmentation des compétences et l'évolution des missions auxquelles les municipalités ont été confrontées au cours de ces années. Si ce changement concernait initialement - dans le prolongement de la reconstruction de l'Après-guerre - un éventail de plus en plus large de services liés aux infrastructures locales (planification et rénovation urbaine, transports publics de proximité, construction de logements, protection de l'environnement), les municipalités ont été, avec l'explosion du chômage depuis le milieu des années 1970, de plus en plus confrontées à des missions de politique sociale et d'emploi. En Allemagne, une part importante des missions administratives est ainsi du ressort des municipalités et des groupements de communes. Les communes assument, d'une part, selon les articles 83 et suiv. de la Loi fondamentale, des missions du Bund et du Land par délégation 
(fonctions déléguées : notamment droit relatif à la déclaration du domicile, contrôle des constructions, questions relatives aux étrangers, protection civile et maintien de l'ordre) ; d'autre part, en vertu de l'article 28, de nombreuses missions relèvent de leur responsabilité propre : prestations sociales et entretien des infrastructures culturelles et de loisirs notamment. La plupart des personnels municipaux sont par conséquent employés dans le secteur des affaires sociales (suivi de près par la fonction publique hospitalière). Depuis les années 1960, les municipalités assument dans ces domaines un nombre croissant d'activités qui relevaient auparavant des Länder.

\section{Dualité des statuts : fonctionnaire et employé}

Avec l'institution de la fonction publique de métier, héritée de l'histoire, l'Allemagne appartient - comme la France - à la tradition européenne des États continentaux (König 2002). Celle-ci se caractérise - à la différence de la culture anglo-saxonne de l' « intérêt public » (voir Pollitt/Bouckaert 2004) - par des statuts juridiques différents entre personnes employées par l'Etat et salariés du privé, les personnels de la fonction publique ayant des droits et des devoirs particuliers. L'administration allemande a toutefois pour spécificité de prévoir traditionnellement, à la différence de la France, une dualité des statuts entre employé/ouvrier d'une part et fonctionnaire d'autre part. Dans son art. $33 \mathrm{GG}$, la constitution définit les " principes traditionnels du fonctionnariat », dont une spécificité nationale - établie dès la fin du XVIIIe siècle - consiste à réserver aux fonctionnaires l'exercice de "prérogatives régaliennes" (voir §2 Beamtenrechtsrahmengesetz - BRRG), ce qui a pour conséquence l'emploi traditionnel de fonctionnaires dans la police, l'enseignement et l'administration des finances. Le statut de fonctionnaire repose sur un rapport de droit public entre l'employeur public et l'agent; il se caractérise par la nomination à vie de l'agent, l'affectation dans une catégorie précise (voir ci-dessous), un régime de retraite spécifique («pension» financée par l'impôt) et un rapport de loyauté particulier envers l'employeur qui exclut traditionnellement le droit de grève comme moyen de lutte sociale. A l'inverse, les employés et ouvriers tombent sous le statut du droit du travail du privé : ils sont embauchés sur la base d'un contrat, peuvent être licenciés de droit, perçoivent une retraite largement alimentée par les cotisations d'assurance sociale qu'ils ont euxmêmes versées («retraite») et peuvent faire grève pour imposer des revendications relatives à leur travail.

Tableau 2 : Caractéristiques distinctives des fonctionnaires et des employés

\begin{tabular}{|lcc|}
\hline \multicolumn{1}{|c}{ Caractéristiques } & Fonctionnaire & Employé \\
inamovibilité & oui & non \\
prise en charge & traitement, pension & salaire, retraite \\
droit de grève & non & oui \\
obligation de loyauté & oui & non \\
définition des conditions de travail & loi & convention collective \\
droit disciplinaire & oui & non \\
recrutement en vue... & ...d'une carrière & ...d'un emploi \\
\hline
\end{tabular}

Source : Bogumil/Jann, 2005, p. 88.

Cette conception dualiste des statuts marque une nette différence entre l'Allemagne et la France, où l'idée d'un statut unitaire définissant les conditions de travail pour tous les 
personnels de la fonction publique est essentielle, même si d'autres types de recrutements - «plus libéraux »-(contractualisation) se développent de plus en plus depuis les années 1980. En Allemagne, la tradition de la fonction publique de carrière est au contraire marquée par l'idée que les fonctionnaires assurent (de manière exclusive) les prérogatives régaliennes et qu'ils doivent de ce fait, contrairement aux fonctionnaires français, se démarquer du «petit peuple»; cela se traduit aujourd'hui encore par une position juridique (privilégiée) particulière (d'une part, une pension financée par l'impôt et l'exemption du régime général de retraites ; d'autre part, l'obligation de loyauté et l'interdiction de faire grève), qui n'a jamais existé sous cette forme pour les fonctionnaires français.

Sur des questions importantes, les statuts des fonctionnaires et employés allemands se sont toutefois rapprochés de jure et de facto, la convention collective des employés du service public (Bundesangestelltentarif: BAT ou TVöD ${ }^{1}$ ), base juridique du statut des employés, étant calquée pour l'essentiel sur celle des fonctionnaires. Ainsi, la structure de carrière des fonctionnaires (catégories supérieure, moyenne supérieure, subalterne, inférieure) s'applique également aux différentes catégories (plus de dix) d'employés définies dans le BAT. En outre, après un certain nombre d'années de service, les employés ne peuvent de fait être licenciés.

Tableau 3 : Personnels de la fonction publique par groupes statutaires et catégories (2002)*

\begin{tabular}{|lcrrrrrr|r|}
\hline \multirow{2}{*}{ Groupe statutaire } & \multicolumn{2}{c}{ Bund } & \multicolumn{2}{c}{ Länder } & \multicolumn{2}{c}{ Municipalités } & Total \\
& en milliers & en $\%$ & en milliers & en $\%$ & en milliers & en \% & en milliers \\
fonctionnaire/juge & $* * 315,2$ & 64,3 & 1244,4 & 57,7 & 175,8 & 12,1 & 1735,4 \\
employé & 98,4 & 20,1 & 787,5 & 36,5 & 925,1 & 63,9 & 1867,6 \\
ouvrier & 76,7 & 15,6 & 124,7 & 5,8 & 346,3 & 23,9 & 569,1 \\
total & 490,3 & 100,0 & 2156,6 & 100,0 & 1447,2 & 100,0 & 4294,1 \\
\hline
\end{tabular}

Source : Statistisches Bundesamt $2003 *$ Hors chemins de fer fédéraux, syndicats de communes et « fonction publique indirecte $»$. $* *$ En incluant les militaires : 185,2.

La dualité des statuts (fonctionnaire/employé) n'a pratiquement plus cours dans les municipalités allemandes - à la différence des Länder et du Bund (Kuhlmann/Bogumil 2006) -, comme le montre le faible «taux de fonctionnarisation » (titularisation) des employés municipaux, en baisse, surtout depuis la réunification. Ce phénomène est lié d'une part à la singularité de l'administration municipale, où les missions dites d' « administration de prestation » sont centrales, tandis que les missions régaliennes (la police notamment dans les services du Land), requérant d'après sa définition traditionnelle le statut de fonctionnaire, sont marginales. D'autre part, les employeurs municipaux de l'Est hésitent à titulariser ${ }^{2}$, ce qui s'explique à la fois par la perspective d'avantages financiers à court terme et par la culture administrative de la RDA, qui

\footnotetext{
${ }^{1}$ Le BAT a été remplacé le $1^{\text {er }}$ octobre 2005 par la nouvelle "Convention collective du service public » (TVöD), qui s'applique pour le moment uniquement au Bund et aux municipalités. Les principaux points de réforme sont les suivants : unification des grilles de rémunération des employés et des ouvriers; éléments de rémunération au mérite (« obligation de redistribution » des bénéfices à partir du $1^{\text {er }}$ janvier 2007) ; évaluation obligatoire du travail par les cadres; promotion au mérite au lieu du principe d'ancienneté. En raison du manque de place, nous n'entrerons toutefois pas dans les détails de cette réforme.

${ }^{2}$ Avec environ $2 \%$, la part des fonctionnaires dans les municipalités de l'Est est encore plus faible que dans celles de l'Ouest.
} 
employait traditionnellement peu de fonctionnaires. Un système à statut unique s'est ainsi établi, dans lequel l'institution (encore dominante aujourd'hui aux autres échelons territoriaux) du fonctionnaire de carrière $(12 \%$ du personnel) tend à disparaître tandis que le modèle contractuel gagne du terrain. Sur ce point, les municipalités se distinguent des Länder et du Bund, pour lesquels les fonctionnaires représentent respectivement 64 et $58 \%$ des emplois.

\section{Voies d'accès et carrières}

La forte décentralisation administrative dans l'État fédéral est, comme il a été dit plus haut, contrebalancée par l'existence d'un droit unitaire fédéral de la fonction publique allemande, dont le Bund fixe le cadre tandis que les Länder adoptent les textes d'application spécifiques (mais largement semblables). Cela signifie que les conditions d'accès à la fonction publique sont réglementées de manière homogène au niveau national. En Allemagne, comme en France, on distingue différentes catégories, correspondant à des qualifications requises spécifiques: inférieure, moyenne, moyenne supérieure et supérieure. Comme le montre le tableau 4, les catégories « moyenne supérieure » et «moyenne » constituent l'ossature de la fonction publique allemande, tandis que la catégorie « inférieure » n'a plus qu'une importance marginale et n'a cessé de diminuer (en 1989, sa part était encore de $48 \%$ ). Cela tient sans doute au fait que, pour un nombre croissant de fonctions, les qualifications et compétences professionnelles requises sont de plus en plus élevées, ce qui se répercute sur le profil de formation et sur la répartition par catégories.

Tableau 4 : Personnels de la fonction publique par catégories $(2002)^{*}$

\begin{tabular}{|lcc|}
\hline \multicolumn{1}{|c}{ Catégorie } & en milliers & en \% \\
supérieure & 689,7 & 14,3 \\
moyenne supérieure & 1469,6 & 30,6 \\
moyenne & 1854,6 & 38,6 \\
inférieure & 96,2 & 2,0 \\
autres** & 699,0 & 14,5 \\
total & 4809,1 & 100,0 \\
\hline
\end{tabular}

Source : Kuhlmann/Röber, 2006 * Emplois à plein temps uniquement. ** Employés hors catégorie + ouvriers

Sont requis pour la catégorie supérieure un diplôme de fin d'études d'une formation universitaire ainsi qu'un stage professionnel (Referendariat). Pour accéder à la catégorie moyenne supérieure, il faut être titulaire d'un baccalauréat (général ou spécialisé) et avoir accompli une formation dans un établissement d'enseignement supérieur court (Fachhochschule-FH). L'accès aux fonctions moyennes est généralement possible après une formation professionnelle dans le système dual (par exemple apprentissage dans un service administratif avec école d'administration intégrée), ce type de formation étant notamment proposé - pour les services administratifs municipaux - par des instituts de formation communaux.

À la différence de la France, qui dispose avec l'ENA (Ecole nationale d'administration) d'une institution centrale de formation des élites administratives, l'Allemagne propose des formations initiales et continues des personnels de la fonction publique décentralisées, en vertu de l'autonomie des Länder dans les domaines de la 
culture et de l'éducation. Le Bund s'est efforcé (en vain, le plus souvent) d'établir des institutions centrales de formation pour la catégorie supérieure; certains établissements d'enseignement supérieur (Verwaltungshochschule) comme Spire, Potsdam, et Constance proposent (parfois en 3ème cycle) des cursus de sciences de l'administration. Mais aucune de ces institutions ne peut prétendre être l'école centrale d'administration de l'élite administrative allemande (Benz/Bogumil,2002, p. 7). Il en va de même pour les formations donnant accès à la catégorie moyenne supérieure, pour laquelle le Bund dispose, comme chacun des Länder, de ses propres écoles supérieures d'administration. La structure fragmentée, non centralisée, du système administratif allemand apparaît aussi dans le domaine de la formation continue. Si l'Académie fédérale d'administration publique (Bundesakademie für öffentliche Verwaltung) organise au niveau fédéral des cursus pour les cadres du service public, il existe aussi, au niveau des Länder, des académies de formation continue gérées par les chancelleries d'État correspondantes notamment en Bavière depuis 1968, ou dans le Bade-Wurtemberg depuis 1986 - qui proposent des formations de cadres sur 14 à 15 mois. Cette situation traduit les efforts faits par les Länder pour former leurs propres élites administratives.

Schéma 1 : voies d'accès à la fonction publique en Allemagne

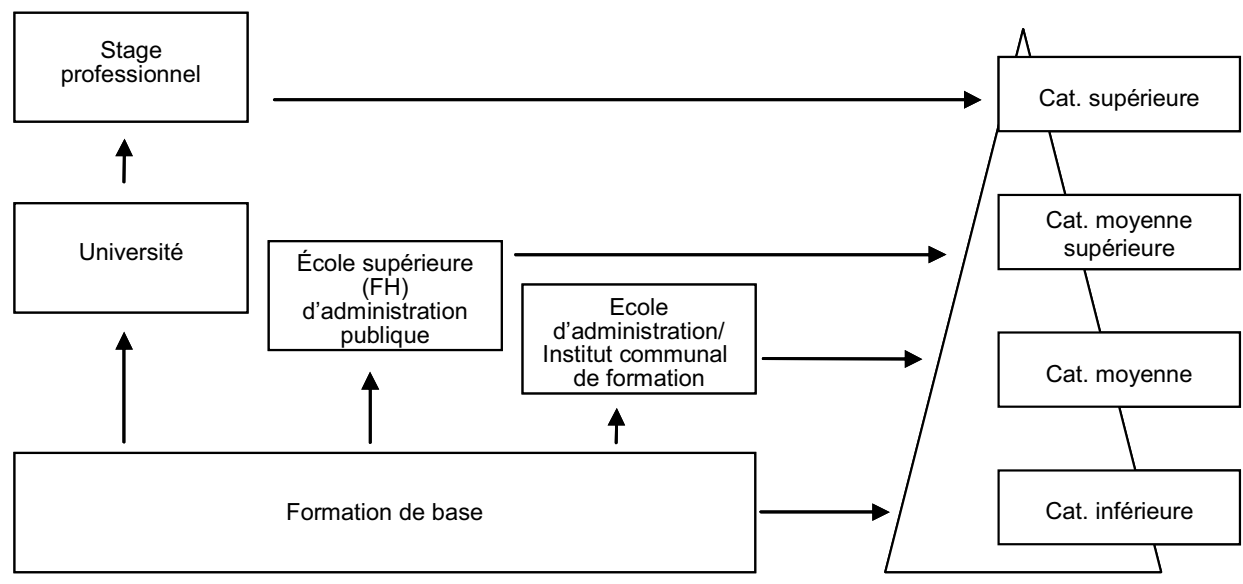

Source : Reichard, 1999, p. 514

\section{Évolution des personnels de la fonction publique après la réunification allemande}

La réunification allemande a fait croître les effectifs de la fonction publique de 1,8 millions de personnes supplémentaires, portant ses effectifs à 6,7 millions en 1991, soit 84 emplois pour 1000 habitants (Bogumil/Jann, 2005). Tandis que les administrations ouest-allemandes réagissaient dès le début des années 1990 aux difficultés budgétaires croissantes par des mesures de consolidation et par une réduction des effectifs (nonrenouvellement de postes vacants en particulier), l'évolution des personnels des administrations est-allemandes prit une tournure radicalement différente après la réunification. Une part considérable du personnel de l'appareil d'État démantelé de la RDA (2,1 millions d'emplois) fut, conformément à l'art. 13, al. 2, du Traité d'unification, transféré aux nouveaux Länder (Wollmann, 1996), si bien que ces derniers se retrouvèrent 
avec 39,0 emplois pour 1000 habitants, soit une densité nettement plus élevée que dans les anciens Länder $(30,2)$. Après la chute du Mur, les effectifs explosèrent aussi dans les municipalités est-allemandes - atteignant 5000 voire 10000 emplois dans certaines grandes villes ${ }^{3}$, lorsque les institutions sociales et culturelles qui relevaient en RDA des organisations d'Etat (ministères, administrations de districts) ou des « entreprises de l'Etat » (VEB), (re)passèrent aux mains des municipalités (furent « recommunalisées »). Finalement, les communes de l'Est se retrouvèrent après la réunification avec des effectifs nettement supérieurs à ceux des municipalités comparables de l'Ouest, si bien que, en 1991, la «densité de personnel » était, avec 41,6 emplois pour 1000 habitants dans les communes de l'Est, deux fois plus élevée que celle des anciens Länder.

Tableau 5 : Evolution des effectifs municipaux sur le territoire fédéral, 1991-2001

\begin{tabular}{|ccccccc|}
\hline Année & \multicolumn{2}{c}{ Municipalités de l'Est } & \multicolumn{2}{c|}{ Municipalités de l'Ouest } & \multicolumn{2}{c|}{ Ensemble de l'Allemagne } \\
& en milliers & pr. 1000 hab & en milliers & pr. 1000 hab & en milliers & pr. 1000 hab \\
1991 & 662 & 41,6 & 1334 & 20,8 & 1996 & 25,0 \\
1994 & 476 & 30,7 & 1330 & 20,2 & 1806 & 22,1 \\
2001 & 309 & 20,6 & 1161 & 17,2 & 1470 & 17,8 \\
évol.1991/2001 (\%) & $-53,3$ & & $-13,0$ & & $-26,4$ & \\
\hline
\end{tabular}

Source : Kuhlmann/Röber, 2006

L'évolution ultérieure des effectifs à l'Ouest comme à l'Est reflète le durcissement de la politique d'emploi et de gestion des ressources humaines sous la pression croissante des contraintes financières. Environ $27 \%$ des postes de la fonction publique ont été supprimés entre 1991 et 2000 , la « densité de personnel » retombant, avec 60 emplois pour 1000 habitants, à son niveau des années 1970. On distingue plusieurs facteurs d'explication : la "privatisation » des chemins de fer et de la poste pendant l' «ère Kohl » (environ 0,8 million d'emplois) d'une part; la réduction des effectifs de la Bundeswehr d'autre part; troisièmement, des réductions drastiques de personnel effectuées par les administrations régionales et municipales, en particulier à l'Est. Les nouveaux Länder ont ainsi réduit leurs effectifs de plus de $25 \%$ pour la seule décennie 1991-2001, en usant en particulier du droit de licenciement exceptionnel prévu dans le Traité d'unification ${ }^{4}$ ou de la possibilité de se séparer, sans que cela pose de problèmes en termes de droit du travail, des personnels considérés en surnombre en "liquidant» les services concernés (réglementation dite de la «file d'attente », "Warteschleifenregelung »). Les municipalités ont elles aussi considérablement réduit leurs effectifs. Tandis qu'ils diminuaient de plus de la moitié entre 1991 et 2001 à l'Est - essentiellement par des accords de rupture avec indemnités et allocations de préretraite ainsi que par des emplois à temps partiel -, les personnels des municipalités de l'Ouest ne bais-

\footnotetext{
${ }^{3}$ En RDA, les municipalités, y compris les villes assumant les fonctions d'un Kreis (Stadtkreise) et les Landkreise, employaient comparativement peu de personnel. Dans les Stadtkreise et les Landkreise, le « cœur » administratif, donc l'administration chargée des missions d'application proprement dites, regroupait entre 250 et 350 emplois, auxquels s'ajoutaient environ 600 autres postes dans les institutions (sociales, culturelles, etc.) associées (Berg, 1996 notamment).

${ }^{4}$ D'après le droit de licenciement exceptionnel, les résiliations peuvent intervenir notamment en cas de qualification professionnelle insuffisante ou d'inaptitude personnelle du candidat, en cas d'effectifs en surnombre ou en cas de liquidation (Abwicklung) d'un service administratif.
} 
saient pour la même période «que » de $13 \%$. La densité de personnel reste toutefois plus élevée dans les municipalités de l'Est que dans celles de l'Ouest.

L'évolution des effectifs depuis 1990 fait apparaître un alignement très net entre les administrations ouest- et est-allemandes. On observe aussi, globalement, une réduction très nette de la densité des emplois publics, retombée à 60 pour 1000 habitants aujourd'hui, contre près de 80 en 1990. En raison des changements structurels décrits plus haut, la part des effectifs employés par le Bund n'est plus que de $13 \%$ (contre $18 \%$ en 1990), tandis que celle des Länder et des municipalités a continué d'augmenter, passant de 49,7 à $51,4 \%$ pour les premiers, et de 32,4 à $35,6 \%$ pour ces dernières.

\section{Comparaison internationale}

Le ratio entre emploi public et ensemble de la population active est souvent cité comme indicateur du poids du secteur public par rapport au privé, et de l'étendue ou de la «minceur » de l'administration publique (Naschold/Bogumil, 2000 ; Pollitt/Bouckaert, 2004). Sur cette base, la République fédérale fait partie des pays de l'OCDE où l'emploi public est relativement peu important. Si l'on prend les chiffres pour l'année 1999, sa part est nettement moins élevée pour l'Allemagne $(15,0 \%)$ que pour les pays scandinaves, et même un peu plus faible que pour les Etats-Unis et la Grande-Bretagne. En revanche, elle est un peu plus forte en Allemagne qu'aux Pays-Bas et sensiblement plus qu'au Japon $(8 \%)$, qui occupe depuis toujours une position spécifique au sein de l'OCDE. Si l'on prend par ailleurs en compte le fait que la proportion d'emplois publics à temps partiel est passée de 6,2 à $25 \%$ entre 1960 et 2000, il est probable que l'Allemagne dispose aujourd'hui comparativement de l'une des fonctions publiques les plus modestes (Derlien, 2002, p. 232).

Tableau 6 : « Minceur » de la fonction publique allemande en comparaison internationale

\begin{tabular}{|ccccc|}
\hline Pays & $\mathbf{1 9 7 0}$ & $\mathbf{1 9 8 0}$ & $\mathbf{1 9 9 0}$ & $\mathbf{1 9 9 9}$ \\
Suède & 20,9 & 30,7 & 32,0 & $* 31,2$ \\
Norvège & 17,7 & 24,1 & 28,6 & $* 30,8$ \\
Danemark & 17,0 & 28,0 & 29,6 & $* 30,0$ \\
Finlande & 12,1 & 17,3 & 20,5 & $* 25,2$ \\
France & & $* * 20,5$ & 20,4 & $* * * 21,3$ \\
Autriche & 13,3 & 18,7 & 19,5 & 18,4 \\
USA & 16,0 & 16,4 & 15,4 & 14,6 \\
Grande Bretagne & 18,1 & 21,2 & 19,5 & 12,3 \\
RFA & 11,2 & 14,6 & 15,1 & 12,2 \\
Pays-Bas & 11,5 & 13,8 & 13,2 & $* 8,3$ \\
Japon & 7,7 & 8,8 & 8,1 & \\
\hline
\end{tabular}

Source : Naschold/Bogumil, 2000, p. 29, avec des actualisations pour 1999 (OCDE, Service de gestion publique, 2001). *Données disponibles uniquement pour 1998. **Données disponibles uniquement pour 1985. ***Données disponibles uniquement pour 1997.

\section{Tentatives de réformes et résultats}

En Allemagne, la critique de la fonction publique et les tentatives de réformes ont une longue histoire. Dans les débats sur une réforme du droit de la fonction publique, du ressort du Bund en vertu des compétences décrites plus haut, on demande depuis 
longtemps la disparition de la traditionnelle dualité des statuts et la création d'un statut unitaire, donc la suppression de la fonction publique de métier inscrite dans l'art. 33 de la Loi fondamentale et de ses «principes traditionnels », qui ne semblent plus avoir de sens aujourd'hui que pour un domaine très étroit de prérogatives régaliennes. D'autre part, la discussion récente vise - comme les débats en France - à faire dépendre la rémunération des agents de leur mérite plus que de leur ancienneté. Il s'agirait en outre de moderniser la fonction publique et de la rendre plus performante en assouplissant le système de carrière (possibilités de promotion) et en renforçant les échanges de personnels entre la fonction publique et les autres secteurs d'activité (recherche, secteur privé). On critique depuis longtemps la trop faible prise en compte de la performance dans la fonction publique : manque d'incitations, notamment dans les systèmes d'évaluation et de rémunération, prise en considération insuffisante des résultats et des objectifs (par le biais par exemple de conventions et d'évaluations d'objectifs). Dans ce contexte, on attend de la réforme qu'elle fasse évoluer les structures bureaucratiques classiques de la fonction publique, centrée sur l'application des lois et des réglementations, vers plus de souplesse et d'intégration de la productivité. Des voix s'élèvent également pour demander des mesures (stratégiques) plus ambitieuses de développement des ressources humaines et un management plus moderne, afin de donner aux personnes des possibilités de participation élargies.

\section{Réforme du statut}

Après l'échec du premier projet de réforme de la fonction publique dans les années 1970, une nouvelle tentative a été faite par une commission du Bundestag en 1997. La loi-cadre correspondante, adoptée la même année par le Bund et destinée à être mise en œuvre par les Länder, comporte deux aspects essentiels, qui concernent toutefois uniquement les fonctionnaires, les conditions de travail des employés et ouvriers relevant de l'autonomie contractuelle : la réforme du statut et l'introduction dans la rémunération d'éléments de mérite.

- La réforme du statut est destinée à faciliter le détachement et la mutation des fonctionnaires. Ceux-ci peuvent désormais être détachés jusqu'à cinq ans sur un autre poste, même contre leur gré, ce qui était quasi impossible auparavant. La loi a également introduit plus de flexibilité en matière de temps et de contrats de travail, en particulier en prévoyant des réglementations pour le travail à temps partiel. Ces dispositions sont de plus en plus utilisées par les employeurs publics pour réduire les coûts de personnel. Il est par ailleurs possible de pourvoir des postes de cadres à l'essai, pour une durée limitée - l'idée de «punir» des personnels peu efficaces en leur refusant le statut de fonctionnaire à vie ayant sa part dans cette nouveauté.

- Concernant l'introduction du mérite dans la rémunération, il faut souligner deux aspects. D'une part, le principe d'ancienneté (donc l'avancement automatique en fonction des années de service) du système traditionnel a été complété par des éléments de mérite. Outre le nombre d'années de service, la performance individuelle d'un agent doit peser pour son changement d'échelon. Le temps nécessaire pour atteindre le dernier échelon a par ailleurs été augmenté, ce qui correspond à la fois à l'objectif de renforcer la productivité de la fonction publique et au souci de réduire les coûts. D'autre part, la réforme introduit la possibilité de «primes d'intéressement versées annuellement » et de compléments de salaire au mérite. Ces suppléments ne peuvent toutefois ni concerner plus de $10 \%$ des agents ni représenter plus de $7 \%$ du traitement de l'échelon correspondant. 
Voyons maintenant comment s'effectue la transposition au niveau régional de ces possibilités offertes par la loi-cadre. Globalement, les Länder ont plutôt peu utilisé les options ouvertes par la loi fédérale (Kuhlmann, 2006b). La plupart d'entre eux ont adopté des lois concrétisant - de manière très variable - la loi-cadre, en particulier en matière de détachement, de mutation et de fonctions de direction à l'essai ou pour une durée déterminée. Mais l'application pratique de ces dispositions dans les administrations reste jusqu'à présent très réservée, voire totalement inexistante. Les collectivités publiques hésitent particulièrement à mettre en œuvre les instruments correspondants pour les primes au mérite et les compléments de salaires. Les dispositions concernant la mobilité ou l'attribution de postes de direction à durée déterminée sont elles aussi peu utilisées. Dans le détail, l'application de la réforme de la fonction publique dans les administrations locales se présente comme suit (Ministère fédéral de l'Intérieur, 2001) :

- Fonctions de direction à l'essai : en 2001, 9 des 16 Länder disposaient d'une législation correspondante; mais, sur l'ensemble du pays, seuls 1760 fonctionnaires s'étaient vu attribuer jusqu'en 2001 de tels postes, soit - pour l'année 2001 - 0,6\% des fonctionnaires de la catégorie supérieure dans les Länder.

- Fonctions de direction à durée déterminée (possible uniquement dans les Länder) : jusqu'en 2001, cette réglementation-cadre avait été transposée dans la législation de 12 des 16 Länder. Mais, jusqu'à cette date, seuls 333 fonctionnaires régionaux s'étaient vu confier des fonctions de direction pour une durée déterminée, soit environ $0,1 \%$ des fonctionnaires de catégorie supérieure employés en 2001 dans les Länder.

- Des règles d'assouplissement en matière de détachement et de mutation ont été introduites jusqu'en 2001 dans tous les Länder; au niveau régional, on compte seulement 1359 cas de détachements correspondant à ces nouvelles dispositions - soit environ $0,1 \%$ des fonctionnaires employés dans les Länder en 2001 - et seulement 197 cas de mutation $(0,02 \%$ des fonctionnaires régionaux en 2001).

- Introduction d'éléments d'intéressement dans la rémunération : jusqu'en 2001, des mesures d'application existaient dans 10 des 16 Länder pour permettre une rémunération au mérite ; dans trois Länder, la transposition était en cours ; les trois autres Länder ne prévoyaient pas d'application des dispositions définies dans la Loi fédérale relative à la rémunération des fonctionnaires (Bundesbesoldungsgesetz).

L'adoption de lois et de règlements est loin de garantir l'application effective par les employeurs publics des éléments de réforme évoqués, ce qui est particulièrement flagrant concernant l'intéressement. Pour toute une série de raisons, les administrations régionales (mais aussi les municipalités ; voir ci-dessous) font très peu usage des primes d'intéressement et des compléments de salaire au mérite. Car, d'une part, cela pose le problème de l'évaluation de la performance: sur quels critères et indicateurs s'appuyer? Comment mesurer et évaluer une bonne/mauvaise performance? Les administrations ne disposent à l'heure actuelle d'aucun système d'évaluation élaboré, et les modes d'évaluation traditionnels n'offrent que peu de points de repère concrets. D'autre part, l'on craint que le versement de primes et de compléments de salaire au mérite ne dégrade l'ambiance de travail, la majorité des agents ne pouvant bénéficier de ces suppléments.

New Public Management - « Neues Steuerungsmodell »

Le débat international sur la nouvelle gestion publique (NGP en français; "Neues Steuerungsmodell - NSM» dans sa version allemande) a gagné l'administration 
allemande dans les années 1990, surtout au niveau des municipalités (Banner, 1991 ; Bogumil/Kuhlmann, 2004 ; Kuhlmann, 2004). À la différence du mouvement NGP international, le NSM a plutôt été perçu comme une alternative aux privatisations et à l'Etat minimal d'inspiration néolibérale - ce qui lui a valu un large soutien au-delà des clivages partisans-, en visant moins les expériences d'ouverture au marché et l'externalisation que les réformes internes (Jann, 2005). L'un des objectifs essentiels du NSM consistait à passer de l'organisation (wébérienne) «bureaucratique classique » des administrations à des formes d'organisation et de gestion tenant davantage compte des contraintes économiques. Il s'agissait d'assouplir les systèmes hiérarchiques rigides, d'intégrer les structures de responsabilité « atomisées », d'abolir le traditionnel clivage entre responsabilité technique et financière et de transformer les services transversaux centralisés (finances, ressources humaines, organisation, etc.) en services avec une fonction interne de mandataire, afin d'en finir avec l'« irresponsabilité organisée » souvent diagnostiquée (Banner, 1991, p. 6) et d'évoluer vers une «structure de direction et d'organisation décentralisée semblable à celle des entreprises » (KGSt 1993a, p. 1620, ici, p. 16). L'objectif était surtout d'axer davantage le pilotage des administrations sur les résultats et les effets des prestations.

Mais, dans le débat sur le NSM, le problème du personnel a d'abord été largement occulté, tandis que les questions de pilotage budgétaire, de gestion financière et d'organisation administrative occupaient le premier plan. C'est seulement par la suite que les partisans de la réforme ainsi que le la $\mathrm{KGSt}^{5}$ (KGSt 1996, p. 7) ont pris conscience du rôle important des employés comme «ressource stratégique » dans le processus de réforme, et de la nécessité de les mobiliser par une participation active, afin de mener à terme le processus de réforme. La modernisation des ressources humaines et de sa gestion est ainsi devenue un sujet de réforme de plus en plus central dans le NSM. La gestion traditionnelle des ressources humaines, à laquelle on reprochait d'être rigide, bureaucratique et hostile au mérite, devait être remplacée par une gestion moderne garantissant plus de souplesse dans l'utilisation du personnel. D'autre part, il s'agissait de créer les conditions permettant de motiver davantage les personnels, en particulier grâce à des systèmes d'incitation financière (primes d'intéressement, système d'avancement au mérite, etc.). Les effets officiellement visés par le NSM étaient donc contradictoires : augmentation de l'efficacité et gains de rentabilité d'un côté ; renforcement de la motivation et augmentation de la satisfaction au travail de l'autre.

Sur la base de données tirées d'une recherche consacrée à l'évaluation de la modernisation administrative dans les communes en Allemagne (Bogumil/Kuhlmann, 2006 ; Bogumil 2006 notamment) ${ }^{6}$, on peut se demander dans quelle mesure les objectifs de politique de gestion du personnel inspirés du NPM/NSM ont réellement été atteints. Les résultats de l'enquête montrent que certains aspects de la réforme ont été expérimentés.

\footnotetext{
${ }^{5}$ La KGSt est une institution intercommunale financée par des contributions des communes adhérentes. Elle bénéficie auprès des municipalités allemandes d'une grande considération et occupe une position très influente dans les consultations relatives à l'organisation et à la modernisation administrative dans les communes. La propagation rapide du NSM tient largement à la campagne de diffusion réalisée par le KGSt au début des années 1980 .

${ }^{6}$ Cette recherche est soutenue par la fondation Hans-Böckler et par la KGSt (période 2004-2006). Dans ce cadre, une enquête exhaustive a été réalisée au printemps 2005 auprès des maires ou Landräte (autorités administratives des Kreise) ainsi que des délégués du personnel de 1565 municipalités. Cette enquête est représentative des communes de plus de 10000 habitants.
} 
$62 \%$ des maires indiquent que des entretiens avec les personnels ont été introduits ; $56 \%$ font état de pratiques de travail en équipe, $47 \%$ d'évaluation des personnels et $35 \%$ de nouvelles procédures de sélection. Dans de nombreuses municipalités favorables à la modernisation, on a recruté des personnels supplémentaires ayant une formation en gestion (36,1\%). Mais, rapportées aux objectifs visés, les activités restent limitées à des procédures assez peu ambitieuses ou au domaine classique de la formation continue. Les éléments centraux du NSM, comme les primes au mérite $(22 \%)$, ou des mesures de réforme plus ambitieuses, telles que les évaluations des cadres $(21 \%)$ et la rotation de postes $(10 \%)$, restent peu répandues.

Graphique 2 : Management des ressources humaines

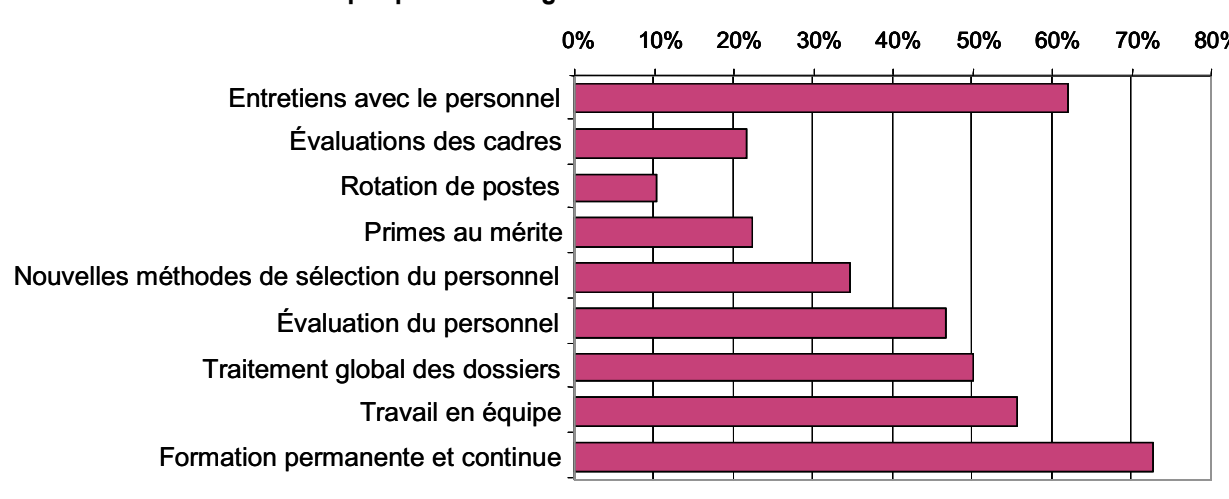

Source : Enquête sur le projet HBS « 10 Jahre NSM »; n=870

Sur les effets des nouveaux instruments de gestion des ressources humaines, on constate un fossé entre les appréciations des maires ("employeurs» municipaux) et celles des présidents des Personalräte (instance élue de représentation des intérêts du personnel). Si les premiers soulignent surtout l'augmentation de la motivation du personnel et l'amélioration de la qualité du travail, les seconds sont nettement plus sceptiques et voient d'abord une augmentation de la charge de travail. Les résultats de l'enquête permettent globalement de conclure que le NSM, avec ses composantes relatives au personnel (pour autant qu'elles aient été introduites dans les communes), n'a pas renforcé la satisfaction au travail. Le personnel redoute au contraire - selon ses représentants - que les contrôles de performance ne se multiplient; il souffre d'une charge de travail croissante.

L'un des dilemmes de la modernisation des administrations réside dans le fait que les personnels sont devenus nettement moins favorables aux réformes dans un contexte de consolidation budgétaire. La situation financière tendue dans les municipalités laisse très peu de marge pour des mesures supplémentaires d'avancement ou d'intéressement. Par ailleurs, les suppressions d'emplois massives au cours des années 1990, en particulier en Allemagne de l'Est, ont non seulement entraîné une intensification considérable du travail, mais aussi accru la peur de perdre son emploi. Dans ce contexte, il n'est guère étonnant que, malgré la participation des personnels au processus de modernisation, leur appréciation de plus en plus négative face aux réformes constitue un problème important. Ainsi, $57 \%$ des maires interrogés ont approuvé l'affirmation selon laquelle une certaine lassitude se serait installée à l'égard des réformes. $52 \%$ indiquent 
que le processus de modernisation est d'abord perçu comme une réduction des effectifs. Ces chiffres sont encore plus élevés (66 à $67 \%$ ) parmi les présidents des Personalräte, particulièrement critiques sur ces questions. Ces résultats peu enthousiasmants s'expliquent notamment par le manque d'influence du personnel dans les différents processus de participation. Ses représentants jugent son influence effective faible dans 70 \% des cas. En outre, la réforme NSM a entraîné la disparition des postes permettant une promotion, un problème affectant particulièrement les femmes (Wiechmann, 2005).

\section{Graphique 3 : Appréciation des effets du développement des ressources humaines}

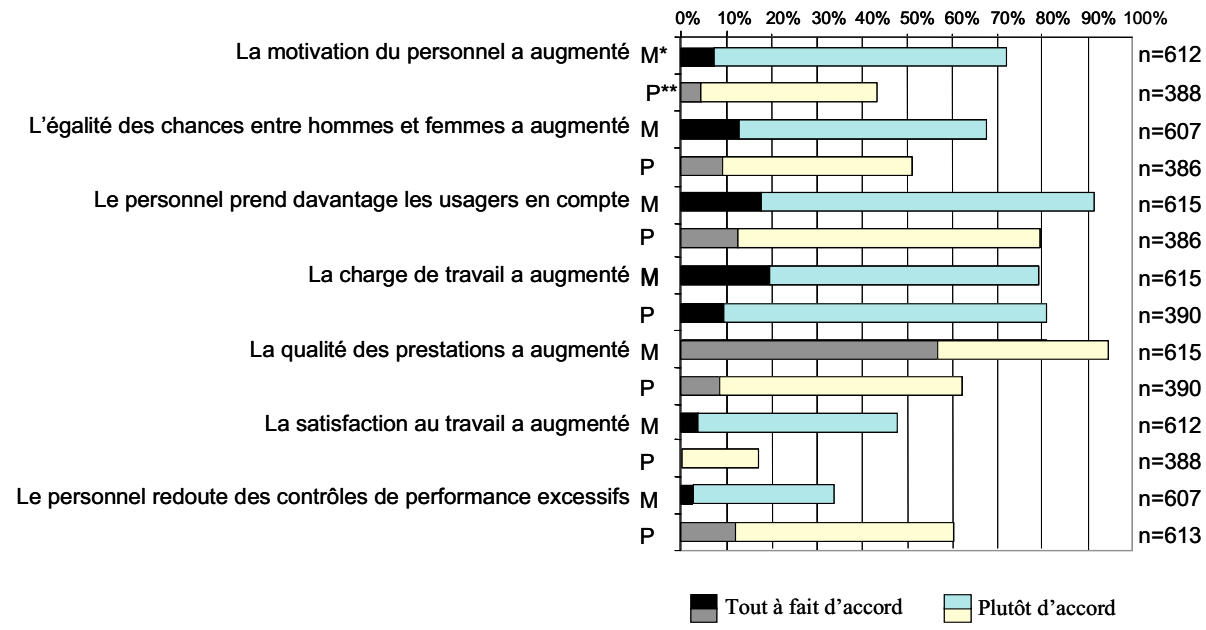

Source : enquête sur le projet HBS : « 10 Jahre NSM »* maires, ** présidents des « Personalräte »

\section{La fonction publique allemande : entre continuité et changement}

Cette contribution a montré que la fonction publique allemande se caractérise à la fois par une force d'inertie et - pour le formuler de façon plus neutre - une continuité dans le temps importantes, qui reflètent l'effet de dépendance (path dependence) à l'égard des traditions historiques, institutionnelles et culturelles, mais aussi l'influence des acteurs ayant intérêt à préserver le statu quo. Cela apparaît d'une part dans la dualité des statuts (fonctionnaire/employé), préservée malgré des critiques récurrentes et plusieurs tentatives de réformes. La portée globalement restreinte de la réforme montre à quel point les processus de changement sont difficiles et laborieux dans la fonction publique. Cette remarque vaut d'un point de vue normatif, c'est-à-dire relativement aux contenus de la loi de 1997, qui ne traitent pas les problèmes structurels fondamentaux (statuts, fonctionnariat). Mais elle s'applique également à la mise en œuvre de la réforme des statuts dans les administrations des Länder : introduction d'éléments d'intéressement et d'incitations à la performance en particulier, mais aussi pistes d'assouplissement du régime statutaire (postes de direction à l'essai/à durée déterminée, mutation/détachement). Au niveau local, les tendances à l'inertie et les résistances à la réforme se traduisent surtout par l'absence d'application à ce jour des composantes importantes pour une nouvelle gestion des ressources humaines axée sur la performance, et par la 
réticence des municipalités à introduire des mesures ambitieuses de développement des ressources humaines (rotations de postes par exemple).

Il ne fait toutefois aucun doute que la fonction publique allemande a été soumise ces dernières années à quelques « ruptures » et changements nets, qui remettent en question au moins en partie les traditionnels phénomènes de " path dependence ». Il faut évoquer en premier lieu les bouleversements survenus à la suite de la réunification allemande, associés, en particulier en Allemagne de l'Est, à des restructurations considérables, à des efforts en termes de qualification, mais également à des mesures très nettes de réduction des effectifs. Ensuite, à partir du milieu des années 1990, l'obligation de réduire le personnel pour des raisons budgétaires a marqué l'évolution de la fonction publique à l'Ouest comme à l'Est, les municipalités étant les plus touchées. Après plus de 30 années d'augmentation continue des effectifs, on observe un changement décisif avec, pour la première fois, des coupes significatives, en particulier au niveau local, entraînant notamment une intensification croissante du travail et - en lien avec le phénomène précédent - une baisse de la motivation et de la satisfaction au travail chez les personnels (encore présents). En ce qui concerne les étapes de modernisation dans la fonction publique, la capacité de changement se traduit par les tentatives de réforme modérées toutefois - du statut en 1997 et par la nouvelle convention collective de 2005 (voir note $\mathrm{n}^{\circ} 1$ ). D'autre part, la propagation rapide du débat sur le NPM a par moments déclenché dans les municipalités allemandes une véritable euphorie à l'égard de la réforme, qui trouve maintenant aussi un écho au niveau du Bund et des Länder. Les effets de ces mesures restent toutefois limités, les instruments économiques du NPM n'étant que partiellement « adaptés » aux besoins spécifiques du service public. Mais les municipalités allemandes auront été les pionnières d'un mouvement de réforme qui laissera une empreinte durable dans la fonction publique et ouvre de nouvelles perspectives.

Traduction de Marie GRAVEY

\section{Indications bibliographiques}

BANNER G., «Von der Behörde zum Dienstleistungsunternehmen - Ein neues Steuerungsmodell für die Kommunen», in : VOP 13(4),1991, p. 3-7

BENZ A., «Institutionentheorie und Institutionenpolitik», in : BENZ A., SIEDENTOPF H., SOMMERMANN K.-P. (éd.), Institutionenwandel in Regierung und Verwaltung. Festschrift für Klaus König zum 70. Geburtstag, Berlin, 2004, p. 19-31

Benz A., Bogumil J. «Civil Servants in a Federal System : The case of Germany.» Communication préparée pour le VIIle congrès de l'AFSP à Lille, septembre 2002

Berg F., Nagelschmidt M., WollmanN H., Kommunaler Institutionenwandel. Regionale Fallstudien zum ostdeutschen Transformationsprozess, Opladen, 1996

Bogumil J., GROHS S., KUHLMANN S., «Ergebnisse und Wirkungen kommunaler Verwaltungsmodernisierung in Deutschland - Eine Evaluation nach zehn Jahren Praxiserfahrungen», in : BoGUMIL J., JANN W., NULLMEIER F. (éd.), Politik und Verwaltung. PVS Sonderheft (i.E.), 2006

BogumiL J., JANN W., Verwaltung und Verwaltungswissenschaft in Deutschland. Einführung in die Verwaltungswissenschaft, Wiesbaden, 2005

BoGUMIL J., KUHLMANN S., «Zehn Jahre kommunale Verwaltungsmodernisierung - Ansätze einer Wirkungsanalyse», in : JanN W., Bogumil J., Bouckaert G., BudÄUs D., HoltKamP L., Kissler L., KuhlmanN S., 
MezGer E., Reichard C., Wollmann H., Status-Report Verwaltungsreform - Eine Zwischenbilanz nach 10 Jahren. (Modernisierung des öffentlichen Sektors, vol. 24), Berlin, 2004, p. 51-64

«Wirkungen lokaler Verwaltungsreformen : Möglichkeiten und Probleme der Performanzevaluation», in : JANN W., RöBer M., Wollmann H. (éd.), Public Management. Grundlagen, Wirkung und Kritik., Wiesbaden, 2006 (sous presse)

Bundesmyinisterium des Innern, Erfahrungsbericht zur Dienstrechtsreform, Berlin, 2001

DRLIEN H.-U., «Öffentlicher Dienst im Wandel», in : KÖNIG K. (éd.), Deutsche Verwaltung an der Wende zum 21. Jahrhundert, Baden-Baden, 2002, p. 229-254

ELLWEIN T., «Verwaltung und Verwaltungswissenschaft», in : Staatswissenschaft und Staatspraxis, cahier 8, 1997

JanN W., «Neues Steuerungsmodell», in: Blanke B., Bandemer S. v., Nullmeier F., Wewer G. (éd.), Handbuch zur Verwaltungsreform, 3e édition, Wiesbaden, 2005, p. 74-84

Jann W., Bogumil J., Bouckaer G., Budäus D., Holtkamp L., Kissler L., Kuhlmann S., Mezger E., REICHARD C., W OLLMANN H., in : «Verwaltungsmodernisierung auf Bundesebene», Status-Report Verwaltungsreform - Eine Zwischenbilanz nach 10 Jahren. (Modernisierung des öffentlichen Sektors, vol. 24), Berlin, 2004, p. 100-111

SCHRÖTER E. (éd.), «Verwaltungsreform und Verwaltungspolitik: Verwaltungsmodernisierung und Policy-Forschung», in:Empirische Policy- und Verwaltungsforschung. Lokale nationale und internationale, Perspektiven Opladen, 2001 p. 279-303

Kommunale Gemeinschaftsstelle (KGSt), «Personalentwicklung im Neuen Steuerungsmodell.» Rapport 6/1996, Cologne, 1996

KÖNIG K., «Zwei Paradigmen des Verwaltungsstudiums - Vereinigte Staaten von Amerika und Kontinentaleuropa», in : KÖNIG K. (éd.), Deutsche Verwaltung an der Wende zum 21. Jahrhundert, Baden-Baden, 2002, p. 393-423

Kuhlmann S (Lorenz), WollmanN H., «Kommunales Dienstrecht und Personal», in : WollmanN H., Roth R. (dir.), Kommunalpolitik. Politisches Handeln in den Gemeinden, 2ème édition, Bonn, 1999, p. 490-512

KuHLMANN S., «Wandel lokaler Verwaltung in Kontinentaleuropa : ein deutsch-französischer Vergleich», in : Bogumil J., Jann W., NullmeIER F. (éd), Politik und Verwaltung. PVS Sonderheft, 2006a (sous presse)

«La Fonction publique en Allemagne : Entre continuité et réformes», in : Association EUROPA (dir.), Entretiens universitaires réguliers pour l'Administration en Europe, L'emploi public en Europe, Presses Universitaires du Limousin (sous presse) Limoges, 2006b

«Evaluation lokaler Verwaltungspolitik: Umsetzung und Wirksamkeit des Neuen Steuerungsmodells in den deutschen Kommunen», in : Politische Vierteljahresschrift, cahier 3/2004, 2004, p. 370-394

KuHLMANN S., BogumiL J., «Civil Service Systems at Subnational and Local Levels of Government : a BritishGerman-French Comparison«, in : Raadschelders, Jos C.N., ToOnEN, ThEO A.J., VAN DER MEER, FriTs M. (dir.), Comparative Civil Service Systems in the 21st Century, 2006 (sous presse)

Kuhlmann S., RÖBER M., «Civil Service in Germany : Between Cutback Management and Modernization», in : Hoffmann-Martinot V., Wollmann H. (éd.), Modernization of State and Administration in Europe: A France-Germany Comparison, Wiesbaden, 2006 (sous presse)

Naschold F., BogumiL J., Modernisierung des Staates. New Public Management in deutscher und internationaler Perspektive, 2ème édition., Opladen, 2000

Pollitt C., BoucKaert G., Public Management Reform. A Comparative Analysis, 2ème édition., Oxford, 2004

ReICHARD C., «Aus- und Fortbildung in der Kommunalverwaltung», in: WollmanN H., Roth R. (éd.), Kommunalpolitik. Politisches Handeln in den Gemeinden, 2ème édition., Opladen, 1999, p. 512-529

WIECHMANN E., «Zehn Jahre kommunale Verwaltungsreform - eine gleichstellungspolitische Bilanz», in : GiP 6/2006, 2006 p. $22-26$

WOLLMANN H., «Institutionenbildung in Ostdeutschland : Neubau, Umbau und schöpferische Zerstörung», in : KaAse M., Eisen A., Gabriel O. W., Niedermayer O., Wollmann H., Politisches System, Opladen, 1996, p. $47-153$ 


\title{
Emploi public en France : état des lieux et perspectives d'évolution
}

\author{
Catherine ZAIDMAN
}

Le poids des effectifs publics dans l'emploi salarié total demeure élevé en France au regard des comparaisons internationales. Encore faut-il s'entendre sur les contours de l'emploi public et les services qu'il prend en charge. Car la question, sous-jacente, est bien celle des objectifs de l'Etat. Cette contribution de Catherine Zaidman, secrétaire générale de l'Observatoire de l'Emploi public, dresse un bilan de l'emploi public en France et s'intéresse à la stratégie engagée face aux enjeux démographiques qui attendent les trois fonctions publiques et aux défis que représente, en termes d'organisation, le double chantier de réforme en cours: réforme institutionnelle d'une part avec la relance de la décentralisation et la poursuite de la politique de déconcentration ; réforme de la procédure budgétaire engagée au 1er janvier 2006, d'autre part, clé de voute de la modernisation de l'Etat.

Cette contribution traitera de l'emploi public en France à partir d'un triple questionnement: qu'est ce que l'emploi public ? Quels sont les défis auxquels les fonctions publiques sont ou vont être confrontées? Quelles orientations retenir pour améliorer l'efficacité des fonctions publiques et garantir ainsi le meilleur service au moindre coût ? Le questionnement portera donc moins sur les modifications apportées aux règles de gestion des personnels, que sur la stratégie globale retenue pour réformer les fonctions publiques et plus particulièrement l'Etat. J'aborderai ces questions, à partir du prisme que sont les travaux de l'Observatoire de l'Emploi public (OEP).

\section{L'Observatoire de l'emploi public}

L'Observatoire de l'emploi public est une structure récente, créée par un décret du 13 juillet 2000 et installée le 19 septembre 2000 par le ministre chargé de la fonction publique. Deux missions lui ont été assignées : la première est d'assurer la transparence sur l'emploi public, la seconde est de favoriser la mise en place de la gestion prévisionnelle des emplois, des effectifs et des compétences (GPEEC), à la fois en aidant à la constitution d'outils, en organisant des échanges d'expériences et en impulsant des études. On verra en quoi cette gestion prévisionnelle de l'emploi, des effectifs et des compétences est au centre du débat sur la réforme de l'Etat aujourd'hui.

Il s'agit d'une instance consultative présidée par le ministre de la Fonction publique et regroupant 43 membres qui représentent à la fois les assemblées parlementaires (Sénat et Assemblée nationale avec respectivement 2 députés et 2 sénateurs), les directeurs d'administration centrale en charge des questions d'emploi public, c'est-à-dire les trois directeurs qui ont la tutelle de la fonction publique ${ }^{1}$ mais également les directeurs

\footnotetext{
${ }^{1}$ La fonction publique en France est décomposée en trois grands secteurs : la fonction publique de l'Etat, la
} fonction publique territoriale et la fonction publique hospitalière. Ces trois fonctions publiques sont régies par 
du budget, les directeurs d'études (le directeur de l'Insee, le directeur de la Prévision ou encore le directeur de la Dares, qui travaillent sur des questions d'emploi public), douze directeurs de personnel de l'Etat, un représentant des directeurs des hôpitaux (Fédération hospitalière de France) ou encore d'organismes chargés à des titres divers de la fonction publique territoriale (CNFPT et CSFPT), des élus de la fonction publique territoriale (un représentant de l'Assemblée régionale, de l'Assemblée départementale et de l'Assemblée des Maires de France) et enfin des représentants des organisations syndicales des trois fonctions publiques.

Il ne s'agit pas d'une structure de négociation mais d'une structure de concertation, de débats et d'échanges, au sein de laquelle on discute de ce qu'est aujourd'hui l'emploi public et de ce qu'il sera demain. Ces échanges doivent permettre à chacun de se forger ses propres convictions, afin d'aller ensuite dans d'autres instances négocier des réformes. L'Observatoire de l'emploi public est animé par un secrétariat, rattaché au Directeur général de l'administration et de la fonction publique.

Il établit tous les ans un rapport ${ }^{2}$, dont le dernier pour la période 2004/2005 présente des données sur la fonction publique selon différents concepts (juridique et économique) et procède à des analyses sur l'emploi public: importance relative des trois fonctions publiques, évolution des effectifs, études sur les caractéristiques des agents (poids des non-titulaires, niveau catégoriel, parité hommes-femmes, qualification-formation), études démographiques, analyse de l'attractivité de la fonction publique, études sur les mobilités des fonctionnaires, la localisation de l'emploi public et sur l'insertion des personnels handicapés. Le rapport se livre également à une présentation des démarches de GPEEC engagées par les trois fonctions publiques, et aborde de ce fait de manière frontale la question de la réforme des fonctions publiques.

\section{Qu'est-ce que l'emploi public ?}

Les travaux de l'Observatoire de l'Emploi public ont d'abord cherché à définir pour la France ce qu'était l'emploi public. On constate en effet que les tableaux de comparaison de l'OCDE ou de l'Union européenne ne définissent pas ce champ de façon identique d'un pays à l'autre, reprenant les données envoyées par les différents pays alors que les contours de l'emploi n'y sont pas les mêmes. A titre d'exemple, j'avancerai deux définitions différentes (une troisième est possible) de ce qu'est l'emploi public en France. Elles permettront de mettre en lumière le fait que, selon la définition retenue, le champ n'a rien à voir. Pour cela, nous avons constitué pour la France un tableau de synthèse de l'emploi public où est positionnée chaque structure (employeur) dans une case et pour laquelle se retrouve l'ensemble des agents qu'elle emploie (voir tableau ci-après).

\footnotetext{
un statut (le statut de la fonction publique), suivi de façon différenciée selon les fonctions publiques par trois directeurs : le directeur de la fonction publique qui anime le réseau des directeurs de personnel de la fonction publique de 1'Etat; le directeur de la DGCL (Direction générale des Collectivités locales) qui lui, au niveau constitutionnel, ne peut intervenir directement dans la gestion de la fonction publique territoriale mais est en charge des textes juridiques régissant les personnels de la fonction publique territoriale; le directeur de l'organisation et de l'hospitalisation, qui est responsable de la fonction publique hospitalière.

2 Les travaux de l'Observatoire sont disponibles sur le site de la fonction publique: Www.fonctionpublique.gouv.fr à la rubrique : Les trois fonctions publiques / Observatoire de l'emploi public.
} 
Tableau 1 : L'emploi public dans l'emploi salarié - classement des employeurs

\begin{tabular}{|c|c|c|c|c|c|c|c|c|}
\hline \multirow{3}{*}{ 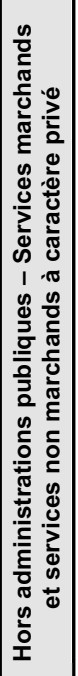 } & \multirow{3}{*}{ 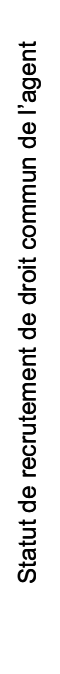 } & 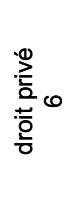 & & & 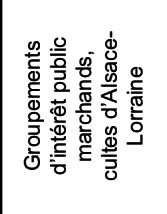 & 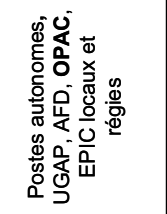 & 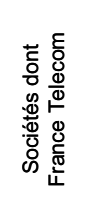 & 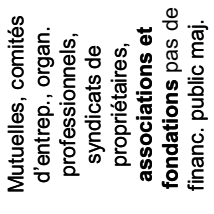 \\
\hline & & 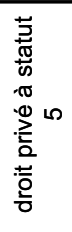 & & & & 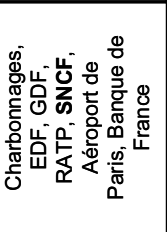 & & \\
\hline & & $\begin{array}{l}\frac{.0}{\overline{0}} \\
\frac{2}{2}+ \\
: \frac{0}{0} \\
\text { 음 }\end{array}$ & & & 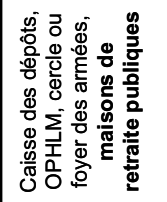 & 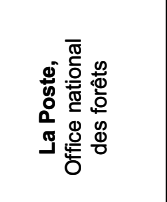 & & \\
\hline \multirow{6}{*}{ 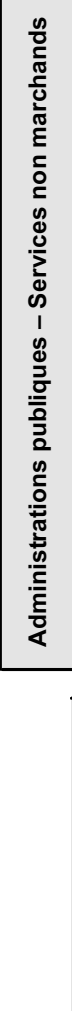 } & \multirow{3}{*}{ 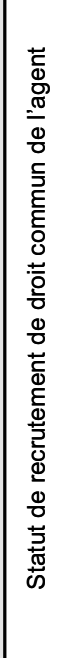 } & $\begin{array}{l}\stackrel{0}{\geq} \\
\frac{2}{2} n \\
: \frac{1}{0} \\
\frac{1}{0}\end{array}$ & & & 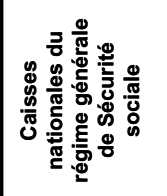 & 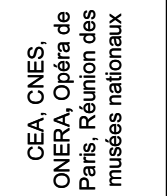 & 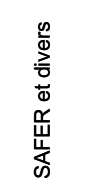 & 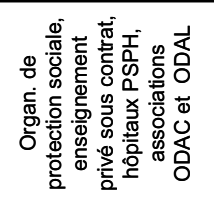 \\
\hline & & 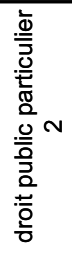 & & & 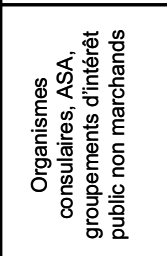 & & & \\
\hline & & $\begin{array}{l}\frac{.0}{0} \\
\frac{0}{2} \\
\frac{0}{0} \\
\frac{0}{0}\end{array}$ & 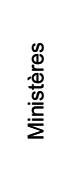 & 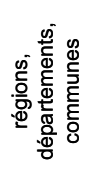 & 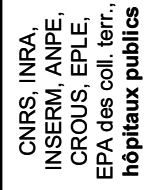 & 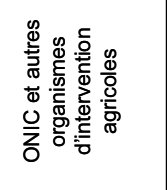 & & \\
\hline & & & $\Gamma$ & $N$ & $m$ & $\nabla$ & 10 & 0 \\
\hline & \multirow{2}{*}{ 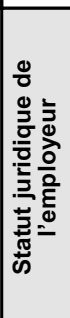 } & 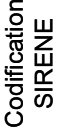 & $\bar{N}$ & N & $\Re \cdot \sigma \stackrel{R}{R}$ & t & 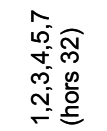 & $\stackrel{9}{\infty}$ \\
\hline & & 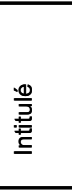 & 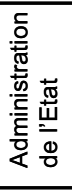 & 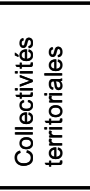 & 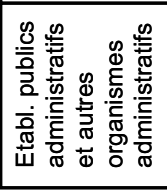 & 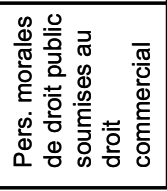 & 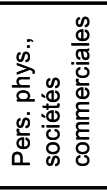 & 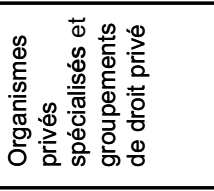 \\
\hline
\end{tabular}


Il s'agit d'un tableau à trois entrées permettant d'établir une classification de chaque structure en fonction de trois critères :

- en colonne majeure : le mode de financement. Il s'agit du critère retenu par la comptabilité nationale. Ce critère permet d'approcher l'emploi public selon une optique "prélèvements obligatoires », il permet de distinguer les employeurs financés majoritairement sur prélèvements obligatoires de ceux majoritairement financés par l'usager ;

- en colonne mineure : le droit de recrutement de l'agent. Ce critère permet de distinguer, pour les services non marchands « droit public, droit public particulier et droit privé » et, pour les services marchands «droit public, droit privé à statut, et droit privé (régi par des conventions collectives) $»$;

- en ligne : la nature juridique de l'employeur. Ce critère permet de distinguer les ministères, les collectivités territoriales, les établissements publics administratifs ou industriels et commerciaux, les sociétés commerciales ou encore les associations de droit privé.

Ainsi, le positionnement d'un ministère apparaît clairement, puisqu'il s'agit d'administration de l'Etat financée majoritairement par les prélèvements obligatoires et dont les agents relèvent du droit public, qu'ils soient fonctionnaires ou contractuels. ${ }^{3}$

Mais il est intéressant, pour bien cerner le terme d'emploi public, de s'intéresser à d'autres types d'exemples. Qu'en est-il ainsi des agents de la SNCF ? La SNCF est un EPIC (établissement public industriel et commercial) financé majoritairement par l'usager et ses agents relèvent du droit privé à statut. Ils ne relèvent donc pas du droit de la fonction publique. Les agents de la SNCF ne sont donc pas des agents de la fonction publique, ni au sens juridique -droit public de recrutement de l'agent-, ni au sens économique -financement majoritaire sur prélèvements obligatoires- (ils ne figurent ni dans la colonne 1, ni dans la colonne 4 du tableau ci-dessus). Les agents de la SNCF sont des agents «à statut» d'une grande entreprise publique financée principalement par l'usager, ce qu'il conviendra d'appeler le service public à la française, au sens où on l'entend généralement.

Autre exemple retenu : celui des maisons de retraite. L'agent de la maison de retraite est un agent de la fonction publique au sens juridique. Son statut relève de la fonction publique hospitalière et il n'y a donc pas d'ambiguitté quand on aborde la question de la réforme de la fonction publique au niveau statutaire : elle concernera également cet agent. En revanche, ce dernier n'est pas un agent de la fonction publique au sens économique, les maisons de retraite étant financées majoritairement par l'usager. En conséquence, il ne figure pas dans l'emploi public pour la comptabilité nationale, c'est-àdire dans le champ généralement retenu quand on aborde la question des prélèvements obligatoires.

Dernier type d'exemple : un agent de la sécurité sociale est-il un fonctionnaire ? Au sens juridique, un agent de la sécurité sociale n'est pas un agent de la fonction publique : son contrat de travail est un contrat de droit privé (régi par les conventions collectives). Il est en revanche un agent de la fonction publique au sens économique puisque la sécurité sociale est financée majoritairement par prélèvements obligatoires. ${ }^{4}$

\footnotetext{
${ }^{3}$ Le contractuel de la fonction publique de l'Etat n'est pas régi par une convention collective de droit privé. Il est régi par des règles juridiques différentes de celles des fonctionnaires mais relève du droit public. En conséquence, un agent de la fonction publique contractuel ne pourra pas faire appel aux prud'hommes.

${ }^{4}$ Le statut de la structure varie toutefois selon qu'il s'agit de l'établissement national (c'est un établissement public administratif) ou d'une structure locale (c'est une association au niveau juridique).
} 
On constate pour autant que certaines conventions collectives s'avèrent parfois bien plus protectrices pour les personnels que ne peut l'être le statut de la fonction publique. Toute analyse doit donc nécessairement être faite au regard de la question posée du droit des agents, ceci étant un point fondamental lorsque l'on se livre à des comparaisons internationales.

Si l'on retient les approches économique et juridique, on aboutit pour la France à deux évaluations de l'emploi public :

- dans une approche de comptabilité nationale, la fonction publique comprend, hors TOM et étranger, au 31 décembre 2003 5,9 millions d'agents (y compris emplois aidés), ce qui représente environ $25 \%$ des salariés de la métropole et des DOM. Parmi ces personnes, seules 3,9 millions sont titulaires ;

- dans une approche juridique, la fonction publique compte, y compris emplois aidés, 5,2 millions d'agents au 31 décembre 2003, ce qui représente $22 \%$ des salariés de métropole et des DOM (et $19 \%$ des salariés et non salariés).

Notons que, bien qu'assez proches, ces deux ensembles ne sont pas composés, comme on vient de le voir, des mêmes cases du tableau présenté ci-dessus.

L'analyse suivante se concentrera sur la fonction publique au sens juridique, c'est-àdire ne prendra en compte, pour reprendre les exemples précédents, ni la SNCF, ni la Sécurité sociale, mais inclura les maisons de retraite. Les 5,2 millions agents qui la composent se répartissent dès lors de la manière suivante : contrairement à l'exemple allemand, c'est la fonction publique de l'Etat qui emploie les effectifs les plus importants $(50 \%)$, contre $31 \%$ pour la fonction publique territoriale et $19 \%$ pour la fonction publique hospitalière (voir graphique ci-dessous). Cette répartition s'explique par l'appartenance à la fonction publique de l'Etat en France des personnels de l'enseignement et de la police. Il faut savoir que sur les 2,6 millions de fonctionnaires et d'agents publics de l'Etat, plus d'un million relève en effet du ministère de l'Education nationale. On voit donc bien là l'impact qu'aurait une éventuelle réforme de la fonction publique basée sur la création d'une quatrième fonction publique, ou encore sur un rattachement de l'enseignement à la fonction publique territoriale : la répartition serait du jour au lendemain considérablement modifiée. Il est important dans le cadre de comparaisons internationales de définir de quels services on parle.

\section{Graphique 1 : Les trois fonctions publiques (effectifs au 31.12.2003 en milliers et en \%)}

Fonction publique hospitalière

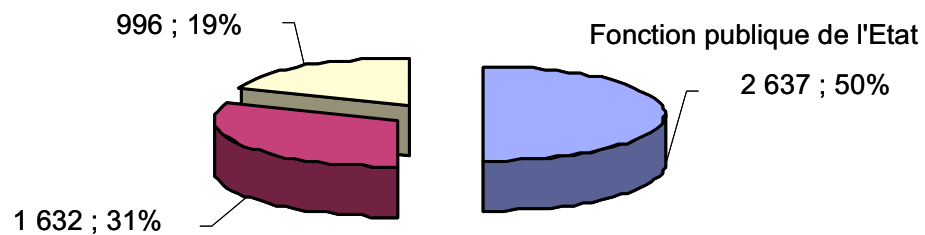

Fonction publique territoriale

\section{Effectif total: 5264 millions dont 5031 millions de titulaires et non-titulaires, 209000 emplois aidés et 23000 militaires volontaires}

Source : OEP - Rapport annuel 2004 - 2005 


\section{Comment évolue l'emploi public ?}

Si l'on s'intéresse maintenant à l'évolution des trois fonctions publiques, on constate que c'est la fonction publique territoriale qui tire la croissance de l'emploi public. C'est effectivement la fonction publique territoriale qui a progressé le plus vite sur la période 1992-2003 (+26,7\%). La fonction publique de l'Etat est celle qui a crû a contrario le moins vite $(+9,2 \%)$, moins d'ailleurs que l'emploi total ( $+11,4 \%$ sur 11 ans).

Parmi les facteurs explicatifs de ces évolutions différenciées entre la fonction publique territoriale et l'Etat on peut citer l'impact de la décentralisation. Celle-ci a fortement modifié la donne et va encore le faire dans les années à venir. La nouvelle loi va en effet entraîner le transfert de 130000 personnes de la fonction publique de l'Etat à la fonction publique territoriale dès 2006 .

Autre facteur explicatif : le développement de missions particulières relevant de la fonction publique territoriale : le secteur de l'action sociale, domaine d'intervention qui a fortement crû ces dernières années, pousse en effet à la croissance de la fonction publique territoriale.

Cette forte croissance des effectifs de la fonction publique territoriale, contrairement à une idée généralement répandue, s'est accompagnée d'une réduction de la part des non-titulaires. Que ce soit dans les ministères ou encore dans la fonction publique territoriale, les agents non-titulaires ${ }^{5}$ sont relativement moins nombreux depuis cette dernière décennie (voir tableau ci-après).

Ainsi, alors que la croissance globale des effectifs totaux dans les ministères s'est élevée à $6 \%$ sur la période considérée, on enregistre une forte baisse des non-titulaires de $16 \%$. Parmi les raisons de cette baisse on peut citer les politiques de déprécarisation des non-titulaires de la fonction publique de l'Etat dans le cadre des plans successifs de consolidation des personnels sur des statuts de fonctionnaire. On peut aussi citer plus largement l'impact de l'accord pris avec les organisations syndicales pour limiter le recours aux non-titulaires dans la fonction publique de l'Etat, avec notamment la mise en place plus systématique de politiques de gestion prévisionnelle des emplois, des effectifs et des compétences.

A contrario, pour les établissements publics nationaux, on observe à la fois des taux de non-titulaires très importants $(62,7 \%$ de non-titulaires dans les établissements publics nationaux) et une forte croissance de leur nombre $(+85,9 \%$ entre 1992 et 2003). L'existence d'établissements publics administratifs dérogatoires permet d'expliquer cette situation. On désigne sous cette appellation les établissements publics qui, par dérogation au statut, ne recrutent que des agents publics non-titulaires : c'est le cas, par exemple, de l'ANPE. Lorsque ces établissements publics dérogatoires croissent de façon importante, le taux de non-titulaires global augmente alors mécaniquement. Dans le domaine de la santé, tous les établissements publics nouvellement créés sont ainsi des établissements publics dérogatoires. Deux ministères importants n'ont d'ailleurs pratiquement que des établissements publics dérogatoires : le ministère de l'Emploi et le ministère de la Sécurité sociale.

\footnotetext{
${ }^{5}$ Notons que sauf exception très particulière de certains établissements publics bénéficiant du droit de recruter des agents de droit privé, les non-titulaires de la fonction publique sont pour l'essentiel des agents de droit public, non fonctionnaires.
} 
En ce qui concerne les communes et leurs établissements publics, les taux de croissance enregistrés (respectivement $+3,7$ et $+36,7 \%$ ), plus faibles pour les non-titulaires que pour les titulaires, font que le poids relatif des non-titulaires est à la baisse.

Dans la fonction publique hospitalière en revanche, on observe une croissance très importante des non-titulaires par rapport aux agents titulaires. Les analyses menées par l'Observatoire de l'emploi public établissent là un lien direct avec la loi sur les 35 heures qui a amené les hôpitaux à recruter massivement des agents non-titulaires pour faire face à la baisse d'activité induite par le passage aux 35 heures.

\section{Tableau 2 : Non titulaires des trois fonctions publiques au 31 décembre 2003} et évolution entre 1992 et 2003

\begin{tabular}{|lcrccc|}
\hline \multicolumn{1}{|c}{ Fonction publique } & \multicolumn{2}{c}{$\mathbf{2 0 0 3}$} & \multicolumn{3}{c|}{ Evolution 1992-2003 } \\
& $\begin{array}{c}\text { non- } \\
\text { titulaires }\end{array}$ & $\begin{array}{c}\text { effectifs } \\
\text { totaux }\end{array}$ & $\begin{array}{l}\text { \% non- } \\
\text { titulaires }\end{array}$ & $\begin{array}{c}\text { évol.des ef- } \\
\text { fectifs totaux }\end{array}$ & $\begin{array}{c}\text { évol. des } \\
\text { non-titulaires }\end{array}$ \\
ministères & 184401 & 2313430 & 8,0 & 6,0 & $-16,2$ \\
EPA nationaux & 144061 & 229921 & 62,7 & 56,7 & 85,9 \\
total fonction publique de l'Etat & $\mathbf{3 2 8} \mathbf{4 6 2}$ & $\mathbf{2 5 4 3 \mathbf { 3 5 1 }}$ & $\mathbf{1 2 , 9}$ & $\mathbf{9 , 2}$ & $\mathbf{1 0 , 4}$ \\
communes, départements, régions & 271991 & 1162490 & 18,8 & 18,3 & 3,7 \\
EPA locaux & 91328 & 359653 & 25,4 & 63,9 & 36,7 \\
total fonction publique terr. & $\mathbf{3 0 9 3 1 9}$ & $\mathbf{1 5 2 2} \mathbf{1 4 3}$ & $\mathbf{2 0 , 3}$ & $\mathbf{2 6 , 7}$ & $\mathbf{1 1 , 7}$ \\
fonction publique hospitalière * & 130135 & 966340 & 13,5 & 14,5 & 95,1 \\
total fonction publique & $\mathbf{7 6 7 9 1 6}$ & $\mathbf{5 0 3 1 ~ 8 3 4}$ & $\mathbf{1 5 , 3}$ & $\mathbf{1 5 , 0}$ & $\mathbf{1 6 , 6}$ \\
\hline
\end{tabular}

Source : Insee, Drees, DHOS. *L'évolution des effectifs de la FPH est calculée hors non-titulaires sur crédits de remplacement non disponibles en 1992 (DGAP, bureau des statistiques, des études et de l'évaluation).

\section{Les défis auxquels sont confrontées les fonctions publiques}

Au-delà de ce travail visant à établir les contours des fonctions publiques, l'Observatoire s'est attaché à préciser les défis auxquels les fonctions publiques sont ou vont être confrontées. Parmi ceux-ci, j'en développerai deux :

- les déséquilibres démographiques entre les régions qui constituent une contrainte forte dans la mesure où les fonctions publiques se doivent d'assurer un égal accès des citoyens aux services publics ;

- le retournement démographique du marché du travail avec le départ massif à la retraite des générations d'après guerre et l'arrivée sur ce même marché de générations moins nombreuses.

Je ne développerai pas dans cette contribution d'autres sujets de préoccupation présentés dans le rapport comme celui de l'égalité hommes/femmes, ou encore de la représentativité des différentes catégories sociales dans la fonction publique française. Il s'agit là de deux questions elles-aussi essentielles qui sont bien évidemment intégrées dans la stratégie actuelle proposée d'adaptation des fonctions publiques.

Le déséquilibre démographique entre les régions constitue une caractéristique majeure de la fonction publique en France. Il s'agit là d'un sujet très important quand on s'intéresse à la question du service à l'usager et à sa proximité. La réflexion sur la nature des services publics de demain va de pair avec la question : quel service pour quel usager? 
Ce que l'on constate, c'est que la fonction publique est inégalement répartie entre les territoires et que certaines régions sont fortement suradministrées : c'est le cas de l'Ile de France qui affiche un taux d'administration des trois fonctions publiques de 9,6\% (taux moyen pour l'ensemble du territoire : $8,1 \%{ }^{6}$ ), ce qui peut sembler normal puisque c'est là que siègent les administrations d'Etat et que se trouve toute la "matière grise " de l'Etat. C'est le cas également de deux autres régions : le Limousin et l'Auvergne, mais cette fois-ci, l'explication est la faible densité de leur population. Cet effet est particulièrement marqué quand on considère la fonction publique de l'Etat : on retrouve alors ce qu'on appelle la "diagonale aride », s'étendant de la région ChampagneArdennes à la région Midi-Pyrénées, où la densité démographique est très faible. A l'opposé, cette diagonale affiche des taux d'administration de la fonction publique de l'Etat supérieurs au taux moyen. Ce phénomène est d'ailleurs encore plus net si on ne considère que l'enseignement : cela signifie que l'enseignement, en assurant un égal accès sur l'ensemble du territoire à l'usager, coûte cher à la collectivité. Mais il s'agit là d'un objectif de l'Etat qui est d'assurer un égal service public à tous et partout. Pour autant, ce facteur très important va jouer lorsqu'on aborde les questions de démographie et de dynamique démographique. Assurer un service public sur l'ensemble du territoire et donc dans des régions de moins en moins peuplées entraîne automatiquement, par personne couverte, une augmentation de l'emploi public. Dès lors, on peut remarquer que la fonction publique de l'Etat, notamment dans sa fonction enseignante, a vu ses effectifs croître, alors même que le nombre d'élèves a baissé au cours des dix dernières années. On pourrait se demander s'il s'agit là de «laxisme » mais lorsqu'on mène une analyse beaucoup plus détaillée, on se rend compte que les effets de répartition géographique ont fortement pesé dans cet effet de ciseau entre la baisse des effectifs d'élèves d'une part et l'augmentation du nombre d'enseignants d'autre part. La question démographique régionale doit donc rester présente à l'esprit lorsqu'on parle d'efficacité publique et de baisse de l'emploi public.

Autre spécificité régionale très marquée: le poids de la fonction publique territoriale. Le Sud de la France (région PACA, Languedoc-Roussillon) a une fonction publique territoriale très forte par rapport au reste de la France, et au final on constate que les fonctions publiques occupent une place importante dans les régions touchées par le chômage.

S'interroger sur le rôle que jouent les fonctions publiques dans le soutien à une région constitue dès lors une autre question fondamentale que l'on devra avoir à l'esprit lorsque l'on abordera la réforme de l'Etat.

Autre questionnement dans le cadre la réforme de l'Etat: la dynamique démographique. Il n'y aujourd'hui absolument aucune incertitude sur cette question. La population active française va baisser dans les dix ans à venir avec les départs massifs à la retraite des générations nombreuses d'après-guerre non compensées par les entrées des jeunes actifs sur le marché du travail. En effet, quel que soit le contexte macroéconomique et institutionnel général retenu, ce retournement est prévisible, seule sa date varie. Si on privilégie un scénario à taux de chômage maintenu constant en projection à $9 \%$ et sans réforme des retraites, c'est-à-dire avec maintien des tendances passées, à conjoncture et législation inchangées (scénario Insee), la population active commencerait à baisser en 2007. Si on se fonde sur un scénario plus porteur misant sur une

\footnotetext{
${ }^{6}$ Emploi public y compris enseignement privé sous contrat et bénéficiaires d'emplois aidés, hors militaires.
} 
baisse régulière du chômage et une stabilisation par la suite à 4,5\% à l'horizon 2015 (scénario COR), à ce moment-là, le retournement se ferait en 2010. Quant à l'impact de la réforme des retraites de 2003 , il ne devrait décaler que de quelques années seulement le retournement inéluctable de la population active.

Or dans ce contexte, la fonction publique française apparaît plus marquée que le secteur privé par le vieillissement de ses agents. En dix ans, la part des plus de 50 ans a ainsi crû respectivement de moins d'un point et de plus de 6 points dans les secteurs privé et public. Elle s'avère dès lors plus élevée dans la fonction publique que dans le secteur privé puisque la part des plus de 50 ans est supérieure de 6,4 points à celle observée dans le privé (respectivement $24 \%$ et $17,6 \%$ en 2002, d'après l'Enquête emploi de l'Insee). La fonction publique connaitra donc le choc démographique plus fortement, plus vite, et avec beaucoup plus d'intensité.

Dans le cas de la fonction publique de l'Etat on observe ainsi un vieillissement très marqué des générations de fonctionnaires titulaires : en dix ans, la part des plus de 50 ans s'est ainsi accrue de presque 10 points, passant de $17,6 \%$ en 1992 à $27,3 \%$ en 2002. C'est là la conséquence des forts recrutements des années 60-70, lorsqu'il s'est agit d'ouvrir l'accès du système éducatif à tous les enfants, avec notamment la mise en place du collège unique. Les très forts recrutements d'un personnel jeune auxquels a alors procédé l'Education nationale pendant une période donnée se retrouvent aujourd'hui : d'ici trois ans, les départs à la retraite vont être considérables dans l'Education nationale. Pour certains ministères néanmoins, la question des départs massifs est déjà derrière eux : c'est le cas pour la Police nationale, dont la majorité des agents est classée en catégorie active et part dès lors plus tôt à la retraite : les recrutements pour remplacer les départs ont déjà commencé. L'analyse montre aussi que les recrutements des dernières années ont également été plus importants dans l'Education nationale. ${ }^{7}$

Ce phénomène se retrouve aussi pour la fonction publique hospitalière avec une pyramide différente (la part des plus de 50 ans s'élevait à $11,5 \%$ en 1992 et à $20,5 \%$ en 2002), et pour la fonction publique territoriale (17,9\% en 1992 et $25,5 \%$ en 2002). Cette dernière a toutefois procédé à un continuum de recrutements tout au long des années passées qui la préserve en partie aujourd'hui de cet effet pointe que l'on a pour la fonction publique d'Etat.

Il faut donc s'attendre à des vagues très importantes de départs à la retraite d'ici 2015. Ceux-ci concerneront $45 \%$ des agents de l'Etat, $41 \%$ des agents de la fonction publique hospitalière et $38 \%$ de la fonction publique territoriale (avant la réforme des retraites). La réforme des retraites ne devrait pas modifier fondamentalement ces chiffres car, s'il est vrai qu'elle permet de réaliser des économies budgétaires puisque les agents diffèrent leur départ, elle ne fait que provoquer un décalage qui ne changera pas fondamentalement les ratios à l'horizon 2015. Ainsi, sans préjuger de l'évolution du nombre des emplois nécessaires à l'exercice des missions des fonctions publiques, les besoins en recrutement devraient être importants dans les années à venir.

Or en vingt ans, les fonctions publiques ont fait de plus en plus appel à des diplômés (voir graphique ci-après) et aujourd'hui, l'Etat absorbe $30 \%$ des jeunes sortant du système éducatif avec une licence. A structure de recrutement inchangée, on estime que

\footnotetext{
${ }^{7}$ Pour une analyse plus précise permettant d'anticiper les effets des départs à la retraite selon les ministères et les catégories d'agents, voir le rapport annuel $2004-2005$ de l'Observatoire de la fonction publique.
} 
les besoins devraient augmenter d'au moins 5 points d'ici 2010. La concurrence entre fonctions publiques et secteur privé risque donc de s'accroître à l'horizon des 10 ou 15 prochaines années et l'on peut s'attendre à des pénuries de main d'œuvre pour les plus qualifiés, alors que le chômage restera élevé pour les non qualifiés. Cet effet structurel qui pose problème pour l'activité économique, constitue donc un vrai sujet de préoccupation pour l'ensemble de la société française.

\section{Graphique 2 : évolution des niveaux de diplôme des salariés âgés de 18 à 35 ans selon le secteur d'activité (public ou privé)}

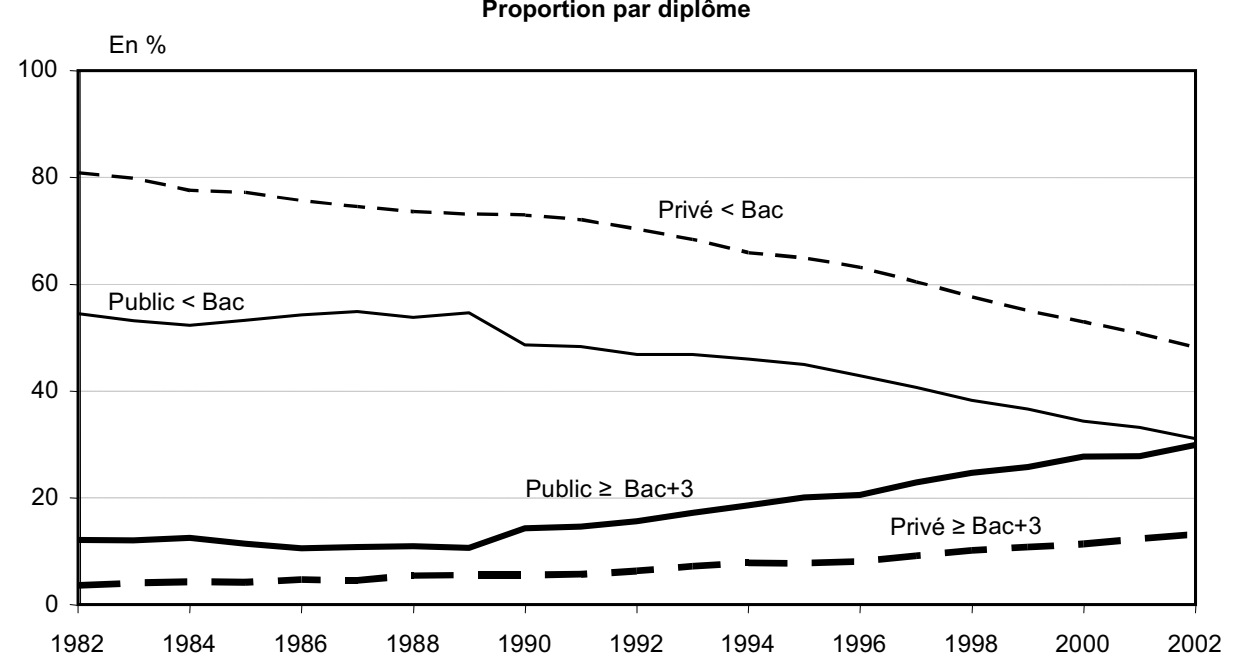

Champ: salariés âgés de 18 à 35 ans.

Lecture: Pour chaque secteur d'activité (public ou privé), les deux courbes représentées correspondent au pourcentage de jeunes (de 18 à 35 ans) recrutés avec un niveau de diplôme égal ou supérieur au BAC+3 ou inférieur strictement au BAC (CAP, BEP, brevet ou aucun diplôme). Pour arriver à $100 \%$, il faut rajouter les jeunes ayant obtenu strictement un diplôme de niveau BAC et BAC+2.

Source : Insee, enquêtes sur l'emploi

\section{Développement des démarches de gestion prévisionnelle des emplois, des effectifs et des compétences}

Pour faire face à ce contexte et améliorer l'efficacité des fonctions publiques, différentes réformes ont été introduites, comme en Allemagne, avec plus ou moins de succès (rémunération au mérite, développement de la mobilité, etc.). Parmi elles figure la mise en place de démarches de gestion prévisionnelle des emplois, des effectifs et des compétences (GPEEC) dans les trois fonctions publiques. Ces démarches, déjà anciennes puisqu'elles ont été initiées dès le début des années 90 , sont restées longtemps au stade du discours et non de l'action. Elles ont été relancées avec la signature du protocole d'accord sur la précarité du 10 juillet 2000 et la création de l'Observatoire de l'Emploi public le 13 juillet. Ces démarches ont été confirmées lors des comités interministériels pour la réforme de l'Etat (CIRE) des 12 octobre 2000 et 15 novembre 
2001, puis renforcées par la circulaire du Premier ministre du 2 décembre 2002. Aujourd'hui, l'idée de mieux calibrer, par la GPEEC, les recrutements de demain, a pris corps. La GPEEC est ainsi devenue au cours de ces cinq dernières années un des leviers de la modernisation de l'Etat.

L'intérêt de cette démarche est relativement clair : il s'agit de partir de la demande exprimée par les citoyens sur le service public de demain pour ensuite proposer les adaptations nécessaires. On constate en effet qu'en France, toute tentative de réformer l'Etat en suivant une approche exclusivement en termes de flexibilité, de performances ou de fermetures de services, ne permet pas de progresser. A contrario, lorsqu'on prend comme point de départ de la réforme la définition de l'Etat demain, des missions et de l'organisation, les chances d'avancer s'avèrent meilleures.

Cette démarche doit notamment permettre de relever quatre principaux défis :

- Répondre aux demandes croissantes des citoyens ;

- Faire face, dans un contexte de départs massifs à la retraite, à une concurrence prévisible accrue sur le marché du travail entre le secteur public et le secteur privé ;

- Maintenir durablement les compétences des agents et leur assurer des perspectives de carrières valorisantes ;

- Améliorer l'efficacité des fonctions publiques et notamment de l'Etat dans un contexte budgétaire contraint.

Au niveau de la fonction publique de l'Etat, trois objectifs précis ont été définis :

- la GPEEC doit permettre à l'Etat «employeur» de disposer des viviers de compétences dont il a besoin pour l'exercice de ses missions.

- la GPEEC doit permettre à l'Etat « garant de l'intérêt général » de ne pas recruter au-delà de ses besoins pour ne pas pénaliser la croissance économique. La démarche de gestion prévisionnelle a donc là pour objet d'améliorer la performance de l'Etat de façon à ce que la satisfaction de ses besoins n'assèche pas, dans les années à venir, le marché du travail.

- enfin, la GPEEC doit accompagner la mise en place de la Loi organique du ler août 2001 relative aux lois de finance, la LOLF, clé d'entrée pour la réforme de l'Etat. Dès 2006, la LOLF devrait constituer un véritable 'big bang' au niveau de l'Etat, faisant bouger considérablement les lignes budgétaires et la gestion des personnels. En effet, le budget de l'Etat sera dorénavant voté par missions et programmes, c'est-à-dire globalement par objectifs, lesquels seront soumis à des comptes rendus d'exécution : pour chacun des programmesactions de l'Etat, toute dépense devra être justifiée au premier euro à l'aide d'indicateurs de résultats et de performances, ce qui changera fondamentalement les lignes au niveau de la fonction publique de l'Etat. Dans le cadre de la mise en place de la LOLF, le Plan ministériel de GPEEC devrait permettre de servir de cadrage à l'allocation des moyens entre programmes et à la définition des politiques globales de recrutement et de formation. Il devrait aussi constituer un instrument de dialogue entre la DRH centrale, les directeurs de programme et, en leur sein, les responsables de BOP (budget opérationnel de programme) en permettant notamment de préciser les orientations en fonction des besoins exprimés par les responsables opérationnels. Il devrait enfin s'accompagner de plans locaux de GPEEC permettant de définir les besoins des services compatibles avec les orientations globales retenues.

La GPEEC se révèle donc avant tout comme étant un outil stratégique aidant à traduire les évolutions souhaitées en emplois budgétaires et besoins en compétences. Elle devrait donc favoriser la définition d'une politique globale en termes de formation et de recrutements : chaque ministère devra pouvoir définir et quantifier ses missions, ses actions, 
ses objectifs et, ce faisant, le personnel dont il a besoin. La GPEEC va donc constituer un élément de dialogue entre le ministère, sa direction des ressources humaines ministérielle, et les directeurs de programme, contraints de rendre compte au Parlement de l'efficacité de la dépense au premier euro, mais aussi avec les directeurs des budgets opérationnels de programme, c'est-à-dire au plus bas niveau de la structure administrative (par exemple une préfecture ou une académie qui vont à leur tout devoir justifier chaque dépense).

Il s'agit également d'un exercice de gestion des ressources humaines (GRH) puisqu'il faut désormais définir, par rapport à un objectif central, quel chemin GRH adopter dans le cadre d'un dialogue social soutenu : autrement dit, chaque ministère est sensé s'interroger sur l'espace de négociation avec les personnels pour atteindre un objectif visé. L'enjeu est de rechercher un scénario de moyen terme "gagnant/gagnant/gagnant»: gagnant pour l'usager, par l'amélioration du service rendu (objectif majeur) ; gagnant pour le personnel qui doit, en cas de baisse de l'emploi, obtenir des améliorations en termes de carrière; gagnant pour le contribuable in fine puisque l'on veut réduire la facture budgétaire.

Enfin, troisième caractéristique, la GPEEC est un exercice technique puisqu'elle nécessite pour sa mise en œuvre des systèmes d'information performants et des outils d'analyse.

A titre d'exemple, le Ministère de l'Intérieur (secteur préfectures) a défini l'an dernier une politique de GRH pour conduire le changement correspondant aux évolutions souhaitées de ses missions telles qu'elles sont présentées dans sa Directive nationale d'orientation. Après avoir analysé l'ensemble des interventions et actions des services et déterminé les moyens en personnel affectés à chacune de ces actions (soutien aux collectivités locales, délivrance des titres de séjour, sécurité, etc.), le ministère a élaboré son plan stratégique à l'horizon de 10 ans. Cette politique concerne tout d'abord la dynamique des emplois et des carrières et s'appuie sur un plan de requalification et de repyramidage des corps. Elle concerne ensuite les politiques de recrutement et la formation. Elle concerne enfin le développement des parcours professionnels, encouragé par l'aboutissement des réformes statutaires: réforme de la filière des services techniques du matériel, homogénéisation de la filière professionnelle des systèmes d'information et de communication, fusion des corps administratifs (administration centrale, préfectures, services de police).

Nous ne détaillerons pas ici les démarches en cours dans la fonction publique hospitalière ou dans la fonction publique territoriale bien que ces deux fonctions publiques mènent elles-aussi des réflexions et des actions similaires sur ces questions (voir rapport 2004 - 2005 de l'OEP).

\section{Indication bibliographique}

L'Observatoire de l'emploi public, Rapport annuel 2004-2005, Paris. La Documentation française, 2006, $198 \mathrm{p}+$ Annexes (CD-Rom) 


\title{
La gestion des ressources humaines dans le processus de réforme des administrations publiques : l'exemple de la Basse-Saxe
}

\author{
Susanne KöNIG
}

Cette contribution a pour objectif d'éclairer, en s'appuyant sur l'exemple du Land de la BasseSaxe, le rôle de la gestion des ressources humaines dans le processus de réforme des administrations publiques allemandes.

Ce n'est à vrai dire pas chose aisée. Une étude précise des concepts de «gestion des ressources humaines » (GRH) et de « développement des ressources humaines » montre par exemple qu'il n'en existe pas de définition homogène. Il faut donc analyser le sens des mots pour pouvoir apprécier avec justesse l'importance de la gestion et du développement des ressources humaines $(\mathrm{RH})$ dans le processus de réforme des administrations publiques. C'est pourquoi la présentation du processus de réforme est ici encadrée par une réflexion sur les problèmes que posent ces concepts. Le développement des $\mathrm{RH}$ en particulier comporte de multiples facettes; les responsables ont l'embarras du choix, s'agissant de sélectionner, dans une "palette d'instruments », les « outils » qui conviennent à leur organisation. L'article décrit ensuite comment le Land de Basse-Saxe a réalisé ce processus de sélection et quelles priorités il a établies. L'entretien entre un collaborateur et son supérieur hiérarchique est supposé avoir un rôle de première ligne, c'est pourquoi nous présenterons ici quelques résultats d'analyses consacrées à cet outil de développement des RH. Un bref résumé sur la GRH et le développement des $\mathrm{RH}$ dans le processus de réforme conclut cet article.

\section{Les défis lancés à la gestion des ressources humaines}

Selon Reichard (2000, p. 180-181), la gestion des ressources humaines regroupe toutes les fonctions managériales relatives aux employés, depuis la définition quantitative et qualitative des besoins jusqu'au licenciement des personnels en surnombre, en passant par le processus de recrutement, le développement, le management et le contrôle de la gestion des ressources humaines. Mais si l'on veut rendre compte des aspects plus élaborés de la GRH, ce concept va au-delà d'une simple liste de fonctions managériales. Les nombreux référentiels et programmes des administrations publiques revendiquent une dimension stratégique, à long terme, de la gestion du personnel, rappelant le Human Resource Management (HRM) nord-américain. Cette expression indique que la personne qui travaille ne doit plus être perçue uniquement comme une unité d'imputation de coût, mais aussi comme un facteur de réussite, qu'il faut encourager et motiver pour atteindre les objectifs de l'entreprise (ou, ici, ceux de l'administration) 
(Oechsler 2000, p. 20). Les employés sont appréciés à leurs postes en tant qu'expert(e)s dont les connaissances et les compétences peuvent devenir un facteur stratégique de performance (Staehle 1991, p. 718). Cette conception a ainsi deux sources d'inspiration : d'une part, l'approche comportementaliste (les collaborateurs comme porteurs de nombreuses aptitudes et compétences) ; d'autre part, l'approche économique (les collaborateurs comme « investissement » et " facteur concurrentiel de l'organisation ») (Staehle 1988, p. 580).

Le HRM n'est pas un concept théorique unique et homogène, mais un amalgame d'approches diverses. ${ }^{1}$ En dépit de multiples variantes, les différents courants présentent toutefois les points communs suivants :

- La gestion du personnel acquiert dans le HRM une importance stratégique propre, et devient une mission de management à part entière (Staehle 1991, p. 719).

- Dans les discours, on souligne que les employés sont un important facteur de succès.

- Le passage du concept traditionnel au concept moderne de HRM peut être illustré de manière caractéristique par les stéréotypes de Compliance et Commitment (Guest 1991, p. 152) : la relation psychologique à l'employeur évolue de la "soumission » à l' "attachement » des employés, les relations entre collaborateurs d'une Low Trust (confiance réduite) à une High Trust (confiance élevée) (voir également Schreyögg/Oechsler/Wächter 1995, p. 1920 ; Fischer 1998, p. 66). Pour créer l'engagement (commitment) et l'entretenir, il faut adresser à l'employé diverses propositions de participation, de communication, d'évolution et de rémunération.

La distinction entre HRM «souple» et «dur», telle que Storey l'a introduite dans le débat (par exemple, 1992, p. 26-27), est relativement peu représentée en Allemagne. La littérature distingue les notions d'approches "instrumentale » et " humaniste » (Goss 1997, p. 12 sv.). La variante «dure » ou « instrumentale» souligne que « la personne est une ressource » et un « facteur de coût». On s'efforce dans cette optique de gérer le facteur main d'oeuvre de manière tout aussi rationnelle que d'autres facteurs de production, conformément à la stratégie et aux objectifs de l'entreprise ou de l'organisation. La performance des employés et les moyens d'augmenter cette dernière sont donc au centre des activités de HRM. Cette interprétation met l'accent sur la gestion des ressources humaines (voir par exemple Storey 1992, p. 26). A notre avis, ces politiques de GRH ne se distinguent guère des considérations purement productivistes inspirées du management scientifique tayloriste.

La variante « souple» de la HRM souligne au contraire la dimension humaine des ressources. L'importance de la stratégie et de la réalisation des objectifs n'est nullement niée. Mais on est manifestement conscient que la main-d'œuvre humaine ne peut faire l'objet d'une "planification rationnelle » totale, qu'elle se distingue fondamentalement des autres facteurs de production (Fischer/Weitbrecht 1995, p. 374). La priorité consiste ici à susciter l'engagement par la communication, la motivation et une autre approche du leadership (Storey 1992, p. 26 sv.). Cette différenciation fait en somme clairement

\footnotetext{
1 Le modèle de référence de la Harvard Business School souligne ainsi surtout la «perspective de management général de la GRH » et l'importance des différents intérêts et parties prenantes (Beer et al., 1985 ; Staehle, 1991, p. 728). Le deuxième modèle américain en matière de GRH a été développé à l'université du Michigan. Ses représentants veulent intégrer la structure d'organisation, la GRH et la stratégie d'entreprise ou mission, les deux premiers paramètres devant être subordonnés à la stratégie (Tichy/Fombrun/Devanna 1982, p. 47). Pour une analyse critique des différents modèles de GRH, voir König 2005, p. 10 sv.
} 
apparaître que le passage de la «gestion traditionnelle du personnel » à la «HRM moderne » peut impliquer pour les employés des chances et des risques différents, selon que ce changement s'accompagne d'objectifs plutôt « souples » ou plutôt « durs » (pour plus de détails, König 2005, p. 15 sv.).

Le concept de GRH, au sens du HRM, s'est imposé depuis longtemps dans la plupart des entreprises du secteur privé en Allemagne; il n'est donc pas surprenant que le secteur public ait lui aussi adopté ce modèle dans sa conception des RH. L'approche « moderne », axée sur la stratégie, se heurte toutefois ici à un modèle ancien de gestion $\mathrm{du}$ personnel principalement basé, encore aujourd'hui, sur une fonction publique traditionnelle. Il existe pour les fonctionnaires, en tant que « loyaux serviteurs de l'Etat », un système de carrière prévisible, presque «figé », de bonnes garanties matérielles d'existence, un statut comparativement rigide et, jusque-là, des incitations à la performance peu nombreuses (ou, de fait, peu utilisées) (Reichard 2001, p. 181 sv.; Tondorf/Jochmann-Döll 2004, p. 428-429.). L'explosion des dépenses imputées aux budgets publics a dans certains cas entraîné de fortes réductions de personnel, d'autres restant sans doute à venir. S'ajoute le désaccord grandissant, récemment devenu flagrant, entre le Bund, les Länder et les municipalités sur la question des conventions collectives : alors que les employeurs s'efforçaient auparavant de «parler d'une seule voix » dans les négociations avec les syndicats et de formuler des revendications communes, les Länder se sont désolidarisés du groupement des employeurs publics en 2005 ; comme ils ne voulaient pas cautionner, à défaut de pouvoir la financer ${ }^{2}$, la politique du Bund et des municipalités, ils mènent désormais, dans leurs domaines de compétences, leurs propres négociations, conduites par la Basse-Saxe. Dans le pire des cas, il y a maintenant une menace de morcellement des conventions collectives, ayant pour conséquence des disparités accrues entre différents groupes de salariés de la fonction publique. Cette situation brouillée, associée à la crise budgétaire (souvent grave), constitue le cadre et la limite d'une « réorientation » de la gestion traditionnelle du personnel dans l'administration publique vers une approche plus «moderne » au sens de la GRH. Ces paramètres sont importants pour évaluer le rôle de la gestion ou du développement des RH dans le processus de réforme de l'administration publique allemande ; la section suivante leur est consacrée.

\section{Gestion et développement des RH dans le processus de réforme}

\section{La gestion des ressources humaines, élément constitutif d'un « modèle élargi »}

Dans le processus de réforme des administrations allemandes, la GRH n'est bien sûr pas un objectif en soi. Elle relève d'un contexte plus large de projets de réformes, qui correspondent largement aux idées de la Kommunale Gemeinschaftsstelle für Verwaltungsvereinfachung (KGSt). Au début des années 1990, la KGSt a lancé en Allemagne, sous le nom de «Neues Steuerungsmodell» (NSM), un mouvement de réformes. Cherchant à organiser les administrations publiques en "entreprises de services », le KGSt a proposé un modèle qui s'est précisé dans les années suivantes, et a retenu

\footnotetext{
2 Sur un total de 4,7 millions de postes dans la fonction publique, plus de la moitié sont affectés aux Länder, seulement 300000 environ au Bund et le reste, aux municipalités. Dans une situation financière parfois difficile, les Länder veulent à tout prix éviter d'augmenter le niveau des traitements et salaires.
} 
l'attention bien au-delà des seules municipalités. Ce modèle de transformation des administrations prévoyait principalement (KGSt 1993, p. 15 sv. ; Jann 2001, p. 85) :

- de constituer une structure de direction et d'organisation décentralisée, semblable à celle des entreprises ;

- de passer d'une gestion de l'imput (par le contrôle des ressources attribuées aux secteurs) à une gestion des « produits » de l'administration, donc une gestion de l'output ;

- de dynamiser cette nouvelle structure par plus de concurrence.

Pour atteindre le premier objectif, le KGSt recommande de piloter l'action de l'administration et l'attribution des budgets sur la base de contrats entre la politique, l'administration et les services. Les résultats, les ressources et l'utilisation adéquate du personnel devraient passer sous la responsabilité de niveaux décentralisés. Il conviendrait en contrepartie d'établir un service central de contrôle de gestion et de pilotage des missions stratégiques (KGSt 1993, p. 18-19). Pour réaliser efficacement le deuxième point, le passage à une gestion par les résultats, le modèle prévoyait la mise en place d'une comptabilité analytique, basée sur le produit, l'objectif étant une gestion constante de la qualité, destinée avant tout à identifier régulièrement les attentes des citoyens et de l'économie (KGSt 1993, p. 22). Enfin, des éléments de concurrence (comparaisons des prestations entre municipalités, benchmarks, comparaisons avec des prestataires externes) devraient contribuer à donner une impulsion à ces nouvelles structures (Jann 2001, p. 86).

On remarque que le thème de la «gestion des ressources humaines " n'est pas explicitement évoqué à ce stade. C'est pourquoi Bogumil et Kuhlmann (2004, p. 53) parlent d'un "modèle noyau », enrichi par la suite de nouveaux éléments. Parmi les compléments ultérieurs importants, il faut évoquer, outre une plus grande attention portée aux usagers et aux éléments de concurrence, la plus grande prise en compte des collaborateurs, à l'aide d'une gestion moderne des ressources humaines. On a progressivement pris conscience que les efforts de réforme, largement inspirés par des modèles de gestion d'entreprise, ne porteraient de fruits à long terme que s'ils pouvaient s'appuyer sur l'approbation et la motivation des salariés, et qu'il ne suffisait pas, pour réorganiser les administrations de manière constructive, de modifier les seules structures : il fallait donner aux collaborateurs les qualifications pour faire face à des besoins nouveaux et les inviter à participer activement aux changements. Cela supposait de nouveaux défis pour le mode de gestion, jusqu'alors traditionnel, du personnel. Les mesures de développement des RH gagnèrent par la suite une importance considérable dans le processus de modernisation (KGSt 2000, p. 7 ; Drescher 2001, p. 55 ; Lorse 2004, p. 195).

\section{Le développement des $\mathrm{RH}$ dans le processus de réforme : l'embarras du choix}

Un développement des ressources humaines prévoyant et anticipant les besoins à venir est l'une des composantes essentielles d'une GRH stratégique. Mais que faut-il entendre par développement des RH? L'expérience montre que les pratiques et les travaux de recherche sont loin d'en présenter une conception homogène (König/Rehling 2005). Les uns en ont une représentation très étroite, assimilant pour l'essentiel développement à «formation ». Les mesures vont alors surtout dans le sens de la formation professionnelle initiale, la formation continue, la formation accélérée et la 
reconversion. Cette approche correspond, dans le tableau 1 ci-dessous, au premier niveau « Développement des RH au sens restreint ». Si l'on en restait là, il consisterait uniquement à programmer des mesures de formation de manière judicieuse, et ne répondrait nullement aux besoins stratégiques. Selon une conception plus large, le développement des RH va plus loin : il s'agit aussi d'identifier celui qui a le potentiel pour prendre en charge d'autres tâches, éventuellement à un niveau supérieur. Le développement des RH inclut dans ce cas (outre la formation) la promotion des personnels jeunes, la planification des successions et des carrières, le recrutement et l'accompagnement des nouveaux collaborateurs, les procédures d'évaluation des résultats obtenus par les collaborateurs, et bien d'autres choses encore. Selon le tableau 1, la forme la plus large de développement des RH associe la formation, la promotion et le développement de l'organisation. Le développement des $\mathrm{RH}$ devrait être assorti d'un contrôle prévisionnel (et non seulement d'un contrôle des coûts et des données antérieures servant d'appui à la gestion).

Tableau 1 : le développement des ressources humaines comme modèle progressif

\section{Développement des RH: « l'embarras du choix »}

\section{Gender Mainstreaming}

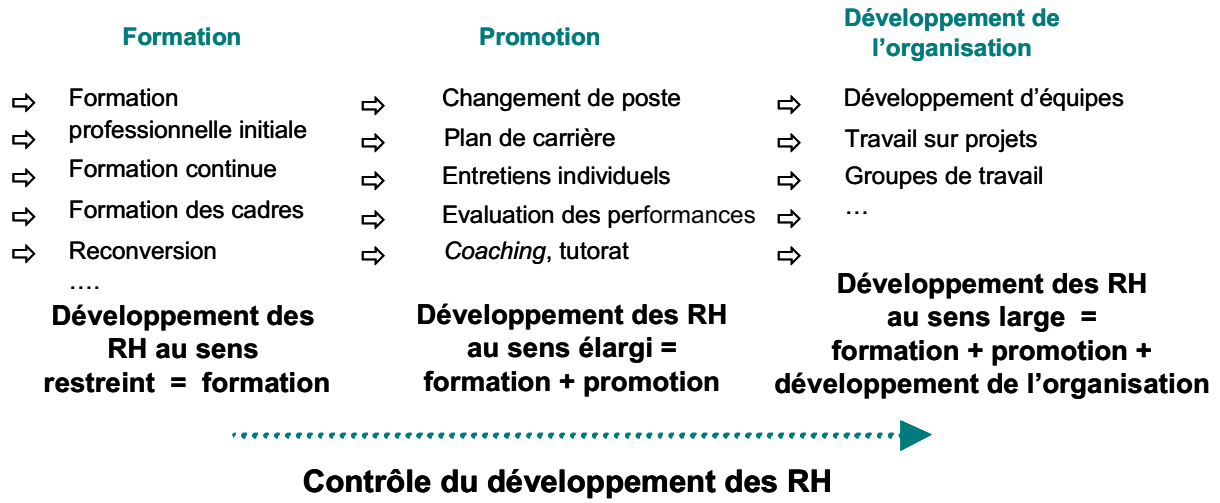

Source : Becker, 2002, p. 6 ; avec des modifications

Dans la fonction publique, le principe du Gender Mainstreaming 3 doit en outre faire l'objet d'une prise en compte transversale. Il a été officiellement établi comme stratégie politique en 1995, lors de la quatrième conférence mondiale sur les femmes organisée sous l'égide des Nations Unies, puis développé par les autorités et la commission européenne ; depuis l'entrée en vigueur du traité d'Amsterdam (1999), il est largement pris en considération dans les administrations publiques et, dans certains cas, dans le secteur

\footnotetext{
${ }^{3}$ Le concept de genre provient de la sphère linguistique anglo-saxonne et désigne (à la différence du sexe biologique) le « sexe social» issu d'évolutions socio-historiques. « Mainstream » est le terme anglais pour le « courant dominant ». Le Gender mainstreaming vise (de manière imagée) à intégrer les questions de parité au courant dominant.
} 
privé. Vouloir respecter les exigences du Gender Mainstreaming suppose d'examiner tous les niveaux, décisions et processus politiques, en se demandant quels sont leurs impacts respectifs sur les hommes et sur les femmes (Jess/Riggers, 2001, p. 187). En Allemagne, le législateur a créé les conditions pour que les décisions politiques prises au niveau du Bund mais aussi de la plupart des Länder respectent le principe du Gender Mainstreaming ; cela s'applique également au développement des RH.

\section{Développement des ressources humaines : l'embarras du choix}

La voie vers une conception homogène du développement des $\mathrm{RH}$, telle qu'elle est présentée dans le tableau 1, n'est qu'une possibilité parmi de nombreuses autres. Les modèles progressifs de ce type semblent toutefois présenter une certaine plausibilité pour les praticiens, et constituer une bonne base pour donner aux acteurs impliqués une idée claire du développement des $\mathrm{RH}$ et de ses objectifs. Cette conception progressive semble d'ailleurs nécessaire, car l'intégration du développement des RH dans les processus de réforme des administrations est organisée de façon variable. Elle intervient souvent par la mise en place de groupes de projets spécifiques, parallèlement à l'organisation ou à la hiérarchie habituelles (pour plus de détails, Rehling, 2006, p. 20 sv.). Ces groupes de projets sont constitués de représentants provenant de services administratifs très variés (par exemple Personalrat ${ }^{4}$, déléguée aux droits des femmes, cadres de services importants, experts du domaine, etc.). Il existe également des groupes de pilotage, qui interviennent en tant que coordinateurs et décideurs. Dans cette configuration, il est important de développer une vision commune du développement des $\mathrm{RH}$; car il paraît pour le moins difficile de vouloir introduire, conduire puis évaluer efficacement les effets d'un projet dont on n'a pas une conception claire.

Enfin, si les acteurs impliqués se sont entendus sur un «langage commun », ils s'accorderont aussi sur des objectifs et mesures communs. Le tableau 1 donne une idée des multiples outils dont dispose théoriquement le développement des $\mathrm{RH}$ dans le processus de réforme. Dans les administrations allemandes qui n'avaient jusqu'alors qu'une gestion du personnel traditionnelle, beaucoup de ces outils (surtout ceux des niveaux les plus élevés) étaient, au début, inhabituels. Le développement des RH se présente ainsi pour les acteurs comme un problème d'embarras du choix, consistant à sélectionner dans une palette d'instruments les outils qui conviendront à leur organisation. D'une administration à une autre, ce problème a été résolu de manière différente, avec des succès divers et à des rythmes variables. Il n'est donc pas possible de faire état d'un stade de développement identique pour le Bund, les Länder et les communes ; Lorse (2004, p. 194) évoque plutôt un " patchwork de considérations conceptuelles ». La section suivante montrera les conséquences que le Land de Basse-Saxe a tirées de cet « embarras du choix ».

\section{L'exemple de la Basse-Saxe : concept-cadre et premières applications}

Dans le Land de Basse-Saxe, la réforme administrative a été introduite à partir de 1994 environ, avec les décisions correspondantes. Selon la déclaration gouvernementale du 23 juin 1994, les principaux projets de réforme devaient comprendre (Rehling, 2006, p. 131):

\footnotetext{
${ }^{4}$ NDT : Instance représentant les intérêts des personnels dans les administrations publiques.
} 
- Une réduction des missions ;

- La diminution des procédures administratives ;

- La motivation des personnels ;

- L'optimisation des moyens mis en oeuvre.

Dans un premier temps, ce n'est pas un vaste plan global qui a été introduit, mais différents projets pilotes. En Basse-Saxe, la réforme présente les spécificités suivantes : l'existence de fortes contraintes budgétaires, une entente relativement précoce avec les syndicats sur la modernisation de l'Etat et une priorité clairement donnée aux mesures de réduction des missions au sein des ministères eux-mêmes (König, 1997, p. 90 ; Rehling, 2006, p. 132).

Trois ans plus tard, le gouvernement régional a adopté un concept-cadre sur le développement des $\mathrm{RH}^{5}$, un service spécifique du ministère de l'Intérieur du Land étant chargé de son élaboration. Par la suite, les compétences ont changé de mains, passant de la chancellerie au ministère des Finances puis (de nouveau en 2000) au ministère de l'Intérieur (pour plus de détails, Rehling, 2006, p. 133). Sur le fond, le concept-cadre a défini les priorités suivantes pour le futur travail de développement des RH (Niedersächsisches Innenministerium 1997a : p. 5 ; Rehling, 2006, p. 134) :

- L'établissement de plans de développement des RH propres aux différentes administrations (Behördenkonzepte) avant la fin de l'année 1999 ;

- L'introduction d'une action de qualification du personnel (sur des questions telles que la prise en compte des usagers, les outils de pilotage empruntés à la gestion des entreprises privées ou encore la gestion de projet) et de formation continue des cadres (au management coopératif ou au contrôle de gestion par exemple);

- La réalisation d'enquêtes de satisfaction des usagers et des collaborateurs ainsi que

- L'introduction à grande échelle d'entretiens entre collaborateurs et supérieurs hiérarchiques d'ici la fin de l'année 1999.

Le gouvernement du Land a octroyé deux fois cinq millions de DM, pour les années 1997 et 1998, à l'expérimentation et à la mise en place de ces mesures. Pour accompagner les procédures sur place, des salariés du Land ont été formés au " conseil en développement des $\mathrm{RH} »^{6}$. Le gouvernement du Land souhaitait ainsi manifester son soutien à ces démarches et créer dans les administrations l'enthousiasme d'un «nouveau départ $»$.

Le gouvernement de Basse-Saxe avait d'emblée accordé une grande importance au contrôle de gestion et à l'évaluation des mesures. Une évaluation centrale et systématique des résultats devait permettre d'avoir un premier aperçu de l'état du développement des RH dans le Land. Dans le cadre d'une enquête exhaustive, les services administratifs du Land ont été interrogés sur leurs activités de développement des RH durant la période $1997-1999^{7}$. Globalement, les responsables en ont conclu que, pour la

\footnotetext{
5 «Concept-cadre» signifie que les administrations subordonnées pouvaient se baser sur les projets du ministère, mais sans que celui-ci puisse imposer une application terme à terme ni aux niveaux inférieurs alors au nombre de trois - de l'administration de Land, ni dans d'autres domaines de compétences du ministère.

${ }^{6}$ Selon les chiffres de septembre 2000, ces formations ont concerné 86 personnes (Niedersächsisches Innenministerium 2002, p. 53).

${ }^{7}$ Municipalités et écoles n'étaient pas comprises.
} 
période étudiée, le thème du développement des RH avait non seulement pris de l'importance, mais qu'il avait, "pour la première fois à grande échelle, entraîné une multitude d'activités » (Niedersächsisches Innenministerium, 2002, p. 6). Les personnes interrogées sur les effets des mesures évoquaient avec prudence notamment une augmentation de la productivité du travail ( $53 \%$ d'approbation), une meilleure conscience des coûts, une hausse de la qualité des prestations et une plus grande volonté d'efficacité ( $52 \%$ d'approbation pour chaque question). Il convient toutefois de nuancer en précisant que seules les directions des services ont été interrogées, si bien qu'on ne peut exclure une déformation dans le sens de ce qui serait « socialement souhaitable». Concernant le détail des mesures, l'enquête indique que :

- Jusque fin 1999, des plans de développement des RH avaient été élaborés dans $46 \%$ des services, la plupart ayant explicitement envisagé de soutenir les femmes ;

- Des plans visant un développement systématique des personnels de direction existaient dans environ un tiers des services ;

- $30 \%$ avaient réalisé des consultations auprès des collaborateurs, à peine $17 \%$ auprès des usagers ;

- Des entretiens individuels structurés avaient été introduits dans $36 \%$ des services.

Outre ce bilan, globalement très positif, les chiffres indiquent donc que des besoins restent à couvrir. Dans le rapport d'évaluation, des objectifs chiffrés ont donc été fixés pour les trois à quatre années suivantes sur la base des résultats actuels. Jusqu'en 2003, le nombre de plans de développement des RH existants dans les administrations devait par exemple passer à $93 \%$, celui des consultations des collaborateurs à $51 \%$ et celui des entretiens entre un salarié et son supérieur hiérarchique à $84 \%$ (Niedersächsisches Innenministerium, 2002, p. 6 ; p. 15 sv.).

Ces chiffres donnent un aperçu significatif de l'état du développement des RH dans le Land de Basse-Saxe. Ils n'indiquent toutefois pas pourquoi, au moment de l'enquête, certains outils étaient nettement moins répandus dans l'administration régionale que les responsables ne l'avaient escompté. Un projet de recherche de l'université Carl von Ossietzky d'Oldenburg s'est penché plus particulièrement sur l'entretien individuel, auquel le plan cadre préalable accordait un rôle privilégié et une importance particulière : le décalage entre l'introduction prévue à grande échelle pour la fin 1999 et le niveau de réalisation (36\% environ) est tout à fait flagrant dans son cas. La section suivante va étudier plus précisément cet outil spécifique. Nous expliquerons d'abord comment l'administration de Basse-Saxe conçoit l'entretien individuel, avant de présenter quelques résultats de recherche.

\section{L'entretien individuel : un outil spécifique}

\section{Le principe de l'entretien individuel salarié - supérieur hiérarchique}

Dans de nombreuses municipalités et administrations de Länder, un rôle particulier est attribué à l'entretien individuel. Il s'agit d'un entretien structuré, préparé, en face à face individuel entre un cadre et un salarié ; il a généralement lieu une fois par an. On le qualifie souvent d'entretien annuel, d'entretien de progression ou d'entretien avec le collaborateur. Il ne doit pas se dérouler spontanément, mais être mené de façon directive, et régulièrement, ce qui le différencie clairement des entretiens " quotidiens » dans le monde du travail. Par rapport au modèle progressif établi selon Becker (voir 
tableau 1), l'entretien ressort de la rubrique «Promotion» (développement des RH au sens élargi). Cet entretien a pour axes essentiels la «coopération», la "gestion et le développement des ressources humaines » et la « détermination d'objectifs ». Contrairement au secteur privé, la fonction publique dissocie explicitement l'entretien individuel de l'entretien d'évaluation. Les cadres ont souvent l'obligation de proposer des entretiens, tandis que la participation des collaborateurs suit dans certains cas le principe du volontariat.

L'introduction d'entretiens va généralement de pair avec des objectifs ambitieux. L'implication de l'employé à travers l'entretien est supposée augmenter la motivation et l'identification à son propre travail, et renforcer la coopération. La « décompression » et "l'assainissement de l'ambiance» ont également une fonction de soupape non négligeable. Mais l'information réciproque et la communication, ainsi que l'ancrage d'une culture du feedback sont particulièrement importants (voir par exemple Breisig/König/ Wengelowski, 2001, p. 36 sv. ; Niedersächsisches Innenministerium, 1997b, p. 5). Les entretiens ne sont néanmoins pas une fin en soi. Ils sont destinés à apporter un accompagnement et un soutien positifs aux efforts de réforme. Le KGSt indique ainsi que l'entretien doit servir «à atteindre les objectifs stratégiques et opérationnels d'une organisation conjointement avec les employés » (KGSt 2002, p. 13). Les entretiens s'ancrent donc (au moins sur le plan théorique) dans le contexte plus large des nouveaux outils de pilotage, en particulier dans le management dit « contractuel ».

Comme il a été dit plus haut, l'entretien constituait en Basse-Saxe une activité prioritaire du plan cadre de GRH. Le ministère de l'Intérieur a établi en guise de référentiel une brochure comportant des conseils de réalisation, reprenant les premières expériences tirées de projets pilotes menés au ministère de l'Environnement ou dans une administration de district (Niedersächsisches Innenministerium, 1997b). Les modules proposés pour un entretien y sont intitulés module de "Coopération", «Missions et environnement de travail », «Possibilités de progression et de développement $\gg .8$ Selon la conception du ministère, une liste de mots-clés peut servir de fil conducteur pour aider l'employé et son supérieur hiérarchique à préparer individuellement l'entretien. Cette liste peut servir à fixer en début d'entretien les thèmes prioritaires. Le tableau 2 montre la liste des mots-clés sous forme condensée.

L'ensemble des thèmes cités doit permettre de déboucher sur des conventions d'objectifs ; c'est pourquoi un module correspondant a été placé en fin de liste. Les conventions d'objectifs doivent y être consignées par écrit, accompagnés de la date de l'entretien et des signatures des deux parties. L'entretien suivant peut ainsi peut ainsi se référer aux objectifs définis l'année précédente ainsi qu'à leur niveau de réalisation (ou aux raisons d'une réalisation inexistante ou partielle).

Comme pour le modèle de la KGSt, les conventions d'objectifs sont qualifiées « d'élément central de l'entretien » et l'entretien lui-même de «condition essentielle à un fonctionnement administratif orienté vers des objectifs». A long terme, le plan du Land de Basse-Saxe s'inscrit, au moins implicitement, avec ses conventions d'objectifs lors de l'entretien, dans une logique de management contractuel, et oriente davantage

\footnotetext{
${ }^{8}$ L'entretien individuel présente en cela des parallèles avec le modèle du KGSt, qui recommande aux municipalités de commencer par ces modules, les piliers étant l'entretien de travail, de définition des objectifs, de coopération ou de développement des RH. Le KGSt recommande en outre de programmer un module sur l'actuel processus de modernisation de l'administration, alors que cela n'est pas prévu dans l'administration régionale de Basse-Saxe (KGSt $1992 ; 2002)$.
} 
l'action administrative sur les objectifs et les résultats : « Il [l'entretien] constitue, avec le principe du « management par objectifs » à mettre en pratique et surtout à l'épreuve, une possibilité de pilotage importante, ce qui en fait une condition de réussite des nouveaux outils de l'administration régionale de Basse-Saxe » (Niedersächsisches Innenministerium, 1997b, p. 5). Dans les faits, la convention d'objectifs dans le cadre de l'entretien ne doit toutefois pas être considérée comme obligatoire mais comme possible ou conseillée.

Tableau 2 : Liste de mots-clés pour aider à préparer et à réaliser l'entretien MVG

\begin{tabular}{|lr|}
\hline Thème : coopérations : & Je souhaite aborder \\
$\bullet$ Flux d'informations & $\square$ \\
$\bullet$ Aide et soutien & $\square$ \\
$\bullet$ Comportement du management & $\square$ \\
Thème : missions et environnement de travail & Je souhaite aborder \\
$\bullet$ Objectifs de travail et priorités & $\square$ \\
$\bullet$ Marges de manœuvre et de décision & $\square$ \\
Thème : possibilités de progression et de développement & Je souhaite aborder \\
$\bullet$ Souhaits et attentes dans le cadre des missions actuelles & $\square$ \\
$\bullet$ Besoins et souhaits en termes de formation & $\square$ \\
\hline
\end{tabular}

Source : Niedersächsisches Innenministerium 1997b, p. 14 ; abrégé

En résumé, l'entretien individuel est sous cette forme un outil plutôt «souple » de développement des RH, dont on escomptait notamment un fort impact sur la motivation des employés. Il est donc étonnant que l'objectif d'une " introduction à grande échelle » avant la fin de l'année 1999 ait été, avec seulement $36 \%$ de réalisation, très clairement manqué. De plus, même dans les services où l'entretien avait été introduit, tous les employés sont manifestement loin d'avoir été touchés, leur part étant parfois même inférieure à $50 \%$. Les raisons de cet échec et les réserves possibles à l'égard de ces entretiens individuels ont été explorées dans le cadre du projet «PerMit» (Personalentwicklung und Mitarbeiter/innengespräche - Développement des RH et entretiens individuels), de l'université Carl von Ossietzky d'Oldenburg. Nous allons maintenant présenter quelques observations tirées des résultats de cette recherche. ${ }^{9}$

\section{Résultats de notre recherche sur l'entretien individuel}

L'enquête ministérielle dans l'administration du Land a apporté des indications sur le degré de diffusion de l'entretien dans la pratique. Qualitativement trop peu alimentée, et s'appuyant uniquement sur les déclarations des directions des services, elle ne permet toutefois que de supposer pourquoi l'entretien n'avait jusque-là pas été introduit aussi largement que prévu dans les administrations de Basse-Saxe. Les bilans n'ont ainsi pour l'essentiel pas indiqué si l'entretien avait réellement été accepté par les employés et par les cadres et s'il était perçu de manière positive, ou s'il avait au contraire rencontré de l'hostilité et des résistances. Le projet PerMit s'était donné pour but d'apporter quelques

\footnotetext{
${ }_{9}^{9}$ J'ai réalisé ce projet conjointement avec Mette Rehling, diplômée en sciences de gestion ; je lui adresse ici toute ma gratitude pour ce travail commun, toujours stimulant, et la remercie de me permettre de présenter certains résultats ici.
} 
éclairages sur ces questions. La sélection des unités analysées a été réalisée en accord avec la fondation Hans-Böckler et le service du ministère de l'Intérieur de Basse-Saxe chargé des questions de développement des RH. L'accès aux services s'est fait à trois niveaux, intégrant à la fois la direction du service, autrement dit la DRH, le Personalrat et la déléguée aux droits des femmes. Les objectifs du projet étaient ainsi d'emblée portés et soutenus par des acteurs internes importants. Des enquêtes ont été réalisées dans sept administrations au total, ${ }^{10}$ dont trois municipalités et quatre administrations de Land. ${ }^{11}$ Les données suivantes s'appuient d'une part sur 234 entretiens en face à face avec des cadres et des employés (barres claires dans le tableau 3), d'autre part sur les réponses aux questionnaires de la ville A (868 questionnaires) et de l'administration régionale 1 ( 88 questionnaires). Cette étude n'a aucune prétention à la représentativité. Mais, dans la mesure où les entretiens de la fonction publique sont à de multiples égards conçus de manière semblable et où il peut y avoir tout au plus de minimes différences de contenu entre les Länder, les observations faites en Basse-Saxe devraient donner une idée claire de la problématique liée à l'entretien. Sur la multitude de résultats, seuls les points suivants ont été retenus ici :

- Le degré de diffusion dans les administrations interrogées, donc le nombre d'employés ayant passé un entretien ;

- L'opinion des personnes interrogées avant et après l'entretien ;

- L'importance des conventions d'objectifs dans le cadre de l'entretien.

\section{Degré de diffusion de l'entretien dans les administrations}

Le tableau 3 fait apparaître de manière synthétique des différences d'accueil d'une administration à l'autre : le degré de diffusion oscille entre 27 et presque $100 \%$. Pour interpréter ces résultats, il faut toutefois prendre en compte la durée depuis laquelle ces institutions travaillent avec cet outil. Au moment de l'enquête, l'introduction de l'entretien dans les administrations Ville $\mathrm{A}$ et Land 1 à 4 datait de cinq à six ans environ. Le «coup d'envoi» de la première série d'entretiens avait été donné environ trois ans avant l'enquête dans le Kreis (district) C. La ville B en était encore à la phase d'introduction, si bien que l'évaluation PerMit présente, au milieu de l'année 2004, un premier bilan intermédiaire.

Ces données seraient à elles seules peu éloquentes, les programmes prévoyant généralement que les entretiens aient lieu chaque année. Il fallait donc approfondir en demandant combien de séries d'entretiens annuels avaient été menées. Dans les administrations de Land 2, 3 et 4, non seulement le degré de diffusion était particulièrement élevé, mais la régularité plus forte : plus souvent que dans d'autres administrations, trois séries d'entretiens annuels voire plus avaient été conduites. Comparativement, la ville A et l'administration de Land 1 avaient, malgré une introduction au même moment, rarement dépassé la deuxième série d'entretiens. Compte tenu de la date d'introduction dans le Kreis $\mathrm{C}$, on aurait pu en être déjà à la troisième série, mais ce scénario ne s'appliquait à aucune des personnes interrogées.

\footnotetext{
${ }^{10}$ Nos moyens financiers et notre temps limités ne nous ont pas permis d'analyser en profondeur un plus grand nombre d'administrations.

${ }^{11}$ Les municipalités avaient développé leurs propres programmes MVG, ceux-ci ne se distinguant toutefois pas fondamentalement dans leurs composantes du modèle des administrations de Land. Il nous semble donc possible de regrouper ici certains résultats.
} 
Tableau 3 : degré de diffusion du MVG dans les administrations

\section{Résultats de l'enquête sur les entretiens}

( $n=234$ entretiens ou 956 questionnaires)

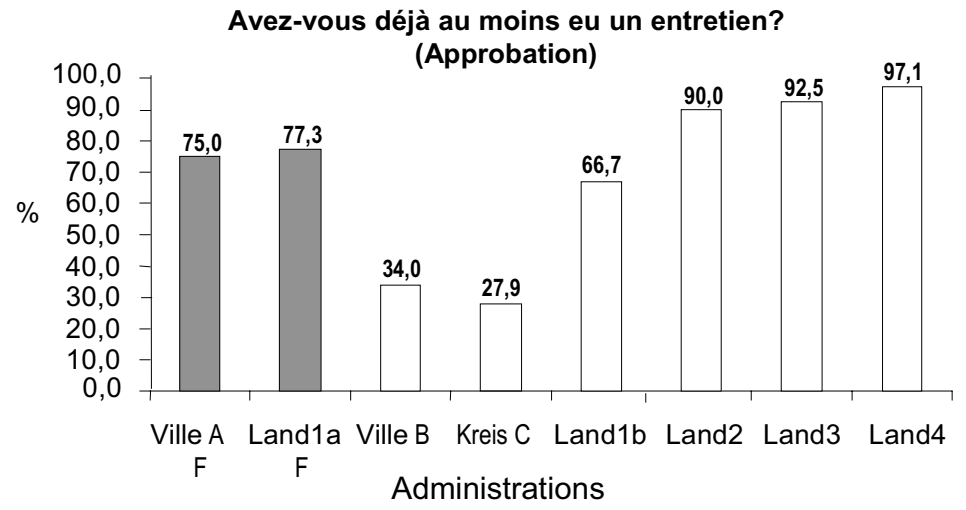

Source : König/Rehling, 2006, p. 42

Avec un outil « souple » et non contraignant tel que l'entretien, il y a de toute évidence un risque d'enlisement pendant ou après la deuxième série annuelle. Il est difficile d'émettre un avis définitif sur les causes possibles ; plusieurs paramètres sont envisageables. Il ressortait de certains entretiens une curiosité éphémère : si l'on était a priori intrigué par ce que cet outil de DRH allait apporter, cette curiosité était satisfaite dès la première série, et il aurait fallu des incitations supplémentaires pour que d'autres entretiens aient lieu. En outre, une partie des cadres ou des employés avaient, seuls ou en concertation, décidé que cet instrument ne pouvait être d'aucune utilité dans leur domaine. Le manque de temps et des problèmes plus urgents en période de réforme ont également pesé. Il faut noter que dans les administrations de Land 2 à 4, qui avaient atteint un nombre de séries particulièrement élevé, la procédure avait été l'objet d'un contrôle centralisé plus fort. L'explication à une diffusion moindre n'est de toute évidence pas à rechercher du côté d'un manque de mesures de formation : dans le Kreis $\mathrm{C}$ (degré de diffusion le plus bas), les collaborateurs et les cadres avaient reçu une formation particulièrement ambitieuse. L'ambiance des entretiens a globalement reçu d'excellentes notes dans toutes les administrations ; la confidentialité des entretiens n'a pratiquement jamais posé de problème. La satisfaction quant aux thèmes abordés est, elle aussi, générale.

Opinion des personnes interrogées avant - après

Quand les employés ont pu être amenés à passer un entretien, leurs expériences sont donc rétrospectivement jugées positives (voir tableau 4). 
Tableau 4 : Opinion sur l'entretien salarié / supérieur hiérarchique avant et après l'entretien

\section{Opinion avant le premier entretien}

( $n=106$ collaborateurs ou 41 cadres)

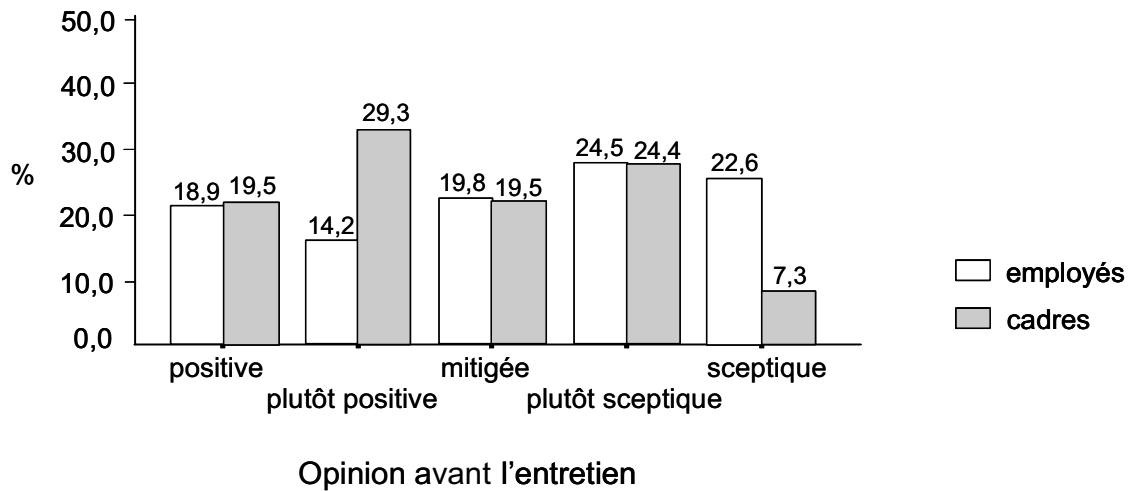

\section{Opinion après le premier MVG}

( $n=108$ collaborateurs ou 42 cadres)

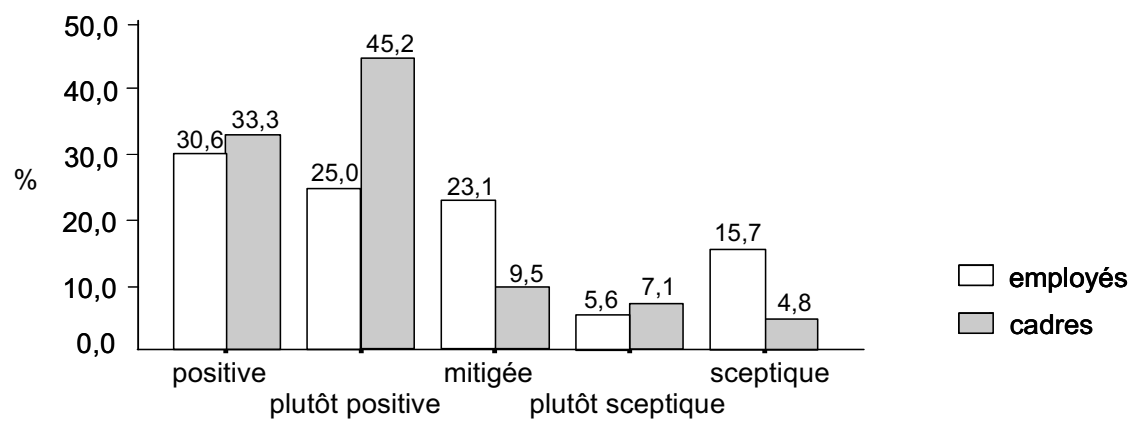

Opinion après l'entretien

Source : König/Rehling, 2006, p. 54

Les déclarations relatives à l'opinion avant le premier entretien indiquent, comme on pouvait s'y attendre, un certain scepticisme - on pouvait s'y attendre car l'entretien restait une procédure nouvelle, inhabituelle. Parmi les personnels, environ 47 étaient initialement sceptiques ou plutôt sceptiques, contre $33 \%$ des cadres. $33 \%$ des employés et $49 \%$ des cadres ont qualifié leur opinion préalable de positive ou plutôt positive. Les autres personnes se sont montrées indécises (position " mitigée ») ou n'ont pas donné de réponse. Après les entretiens, environ $21 \%$ des employés et $12 \%$ des cadres restaient tendanciellement sceptiques. Mais on observe globalement une opinion assez positive (au total, plus de $55 \%$ des employés et près de $80 \%$ des cadres avaient une opinion positive ou plutôt positive). 


\section{Importance des conventions d'objectifs dans le cadre des entretiens}

La convention d'objectifs devrait, selon la vision de la KGSt et du ministère de l'Intérieur de Basse-Saxe, être un élément prioritaire et mener progressivement l'administration à répondre à des objectifs précis. Les municipalités ayant participé à l'enquête s'étaient toutefois sciemment éloignées des recommandations de la KGSt, utilisant, au lieu du concept de «conventions d'objectifs », la formulation non contractuelle de «conventions » ou d'《accords $»^{12}$. Aucune véritable volonté de pilotage n'y était liée. Ce constat vaut d'ailleurs - indépendamment du choix des termes - pour la majorité des administrations étudiées. Seule une administration s'efforçait de s'ancrer à long terme dans une logique de management contractuel, sans vraiment y parvenir car les contrats avaient été passés avec le ministère concerné trop tard pour être établis suffisamment dans le détail. Sur la base des résultats tirés des entretiens préparatoires, on pouvait considérer que tous les termes (conventions d'objectifs, conventions et accords) désignaient principalement la même idée : introduire, comparativement à des arrangements « seulement» oraux, une notion d'engagement. Cela nous autorise à utiliser dans ce qui suit ces différents termes comme quasi-synonymes.

Il a d'abord été demandé si, dans le cadre des entretiens, des changements semblaient nécessaires en termes de coopération, de missions et d'environnement de travail ou de développement des RH. Dans trois entretiens sur quatre, des besoins avaient été identifiés, ce qui laissait présager un potentiel suffisant pour des conventions d'objectifs. La moitié des personnes interrogées indiquait aussi avoir déjà passé des conventions d'objectifs (ou, selon les termes employés dans l'administration, des accords). Les trois quarts des personnes estimaient en plus qu'il leur avait été plutôt facile de parvenir à de telles conventions dans un entretien en face à face. Il apparaissait néanmoins que plus de $40 \%$ des personnes interrogées y avaient de manière générale renoncé. Les personnes interrogées, notamment les personnels de l'administration du Land, avaient manifestement une position très ambivalente sur cet outil. Les conventions d'objectifs définies dans le cadre de l'entretien semblaient curieusement plus importantes pour les employés que pour les cadres, une configuration inhabituelle. La grande dispersion des résultats (se traduisant par un écart-type important) a souligné cette impression d'ambivalence. Les réponses font apparaître que des accords (sur des objectifs ou non) n'ont eu lieu régulièrement que dans environ $40 \%$ des entretiens ; près d'un quart des personnes interrogées était même d'avis que les entretiens ne mènent pas du tout à des accords concrets. Globalement, la "culture d'engagement » des accords ou des conventions d'objectifs semblait très variable selon les administrations. Dans certains secteurs, cet outil était pratiquement déjà établi ; dans d'autres, il restait des réserves importantes quant à son sens. Le contenu et la qualité des conventions d'objectifs passées n'ont en tout cas pas pu être étudiés dans le cadre de cette évaluation.

\section{Bilan intermédiaire de la conduite d'entretiens}

Les projets soulignent très souvent l'importance de l'entretien comme outil essentiel de développement des RH. Quel éclairage apportent les résultats empiriques - présentés ici de manière condensée ? Il semble être un outil tout à fait adapté pour renforcer la

\footnotetext{
${ }^{12}$ Les acteurs souhaitaient même établir une distinction claire avec les « vraies » conventions d'objectifs, ces
} dernières étant passées à d'autres niveaux (par exemple celui de l'équipe). 
motivation, la communication et la coopération. Lorsque des accords sur l'évolution professionnelle avaient pu être conclus malgré la situation difficile des administrations dans le processus de réforme, la satisfaction des employés semblait élevée, ce qui est tout à fait compréhensible. D'un point de vue plus stratégique, des actions ou remaniements semblent cependant nécessaires :

- Sous sa forme actuelle, l'entretien n'ouvre pas encore la voie à un «management par objectifs » dans les administrations publiques allemandes ;

- Les questions relatives à la réforme administrative et le rôle des personnes interrogées dans des processus de travail en évolution (des sujets « brûlants ») ne font pas l'objet d'entretiens ;

- Comme il n'y a dans l'entretien pratiquement aucune harmonisation explicite entre l'évolution à venir des besoins et les qualifications, et que l'entretien semble parfois isolé d'autres outils, les potentiels de développement prévisionnel (au sens d'une GRH stratégique) ne sont pas exploités.

En raison de ces résultats, les entretiens semblent pour l'instant peu à même d'ancrer les administrations dans une logique de management contractuel ou de servir de base à un accord sur la rémunération dans la fonction publique..$^{13} \mathrm{Il}$ y a donc un décalage entre le plan théorique et la réalisation pratique, si bien que des clarifications ultérieures apparaissent nécessaires. ${ }^{14}$

CET ARTICLE VISAIT à apporter un modeste éclairage sur le rôle de la gestion et du développement des RH dans le processus de réforme de l'administration allemande. Nous avons ainsi brossé à partir du cas de la Basse-Saxe un large tableau des différentes stratégies possibles, tantôt « dures » tantôt « souples » de développement des ressources humaines, illustrant ainsi la variété des choix et des instruments qui s'offrent aux décideurs du changement. L'exemple particulier de l'entretien individuel a montré que des projets de développement des RH très élaborés « en haut lieu » se heurtent dans la pratique à différents problèmes de réalisation. Mais, comme chaque instance est finalement elle-même seule responsable de la mise en œuvre sur le terrain, elle ne peut escompter que peu de soutien concret du niveau central. La réorientation de la gestion du personnel, d'une définition traditionnelle à une GRH moderne, doit souvent être effectuée avec les «moyens du bord », ce qui représente une tâche énorme pour chaque administration. Selon un récent sondage, seul un tiers d'entre elles se sent très bien ou bien armée face aux défis du changement (Drescher, 2005, p. 16). ${ }^{15}$

Le Bund n'a pas ouvert la voie en la matière : il a fallu attendre 1999 pour qu'il prenne la décision de programmer l'introduction à grande échelle de plans de dévelop-

\footnotetext{
13 Tondorf, Bahnmüller et Klages (2002) distinguent dans le domaine des administrations publiques trois types idéaux (théoriques) d'objectifs : 1. Des objectifs dans le MVG en guise d'alternative à l'évaluation des performances; 2. Des objectifs comme outil de pilotage (par exemple dans le cadre d'un management contractuel) ; 3 . Une rémunération liée aux objectifs.

${ }^{14}$ Après l'évaluation partielle, le ministère de l'Intérieur de Basse-Saxe de l'époque (2002 : Berichtsteil II, p. 27) tenait par exemple encore à ce que les MVG se développent en un système comportant «des conventions d'objectifs, contractuelles et contrôlables, entre l'employé et son supérieur hiérarchique ».

15105 administrations allemandes ont participé à cette enquête. Les personnes interrogées ont cité comme principaux enjeux à venir : 1. Renforcement de la compétence de direction; 2. Evolution financière/suppressions de postes ; 3. Maintenir la motivation des employés ; 4. Conséquences de l'évolution démographique ; 5. Appliquer les nouvelles conventions collectives (pour plus de détails, Drescher 2005, p. 14).
} 
pement des RH dans ses services (Lorse, 2004, p. 194). Ce sont plutôt les municipalités qui ont un rôle précurseur. Les efforts de modernisation des Länder s'avèrent variables en termes de rythme comme de volonté. Au niveau régional, on travaille souvent avec des projets qui présentent un caractère pilote. Globalement, ce processus est plutôt qualifié de modernisation par adaptation (Reichard 2004, p. 88 sv. ; p. 98-99). Le personnel des Länder représentant des effectifs comparativement importants (voir note $\mathrm{n}^{\circ} 2$ ), la grande difficulté, dans une situation budgétaire parfois précaire, consiste à gérer des missions croissantes avec de moins en moins de personnel. Sur ce point, les efforts de modernisation sont fortement masqués par des mesures d'économie et des réductions d'emplois.

Les remarques suivantes valent probablement au même titre pour le Bund, les Länder et les municipalités : dans le processus de réforme, des éléments empruntés à la gestion et la notion d'efficience économique ont la priorité sur des paramètres plus « souples » comme la gestion des ressources humaines ou son développement. Cela ne signifie pas qu'une «ligne dure » de GRH soit d'emblée visée. Mais, face à une pression budgétaire croissante, il est difficile pour les partisans d'une variante « souple » de la GRH de mettre leurs idées en pratique. On leur demande de démontrer précisément les effets et succès des mesures introduites. Pour les employés, cette juxtaposition d'éléments durs et souples émet ce que l'on appelle des messages contradictoires ou ambivalent : ils se demandent s'ils seront finalement les gagnants ou les perdants de la réforme.

\section{Traduction de Marie GRAVEY}

\section{Indications bibliographiques}

BeCKer M., Personalentwicklung : Bildung, Förderung und Organisationsentwicklung in Theorie und Praxis, 3ème édition, Stuttgart, 2002.

Beer M., Spector B., Lawrence P., Mills D. Quinn, Walton Richard E. (éd.), Human Resource Management : A General Manager's Perspective. Text and Cases, New York ; London, 1985

BoGUMIL J., KUHLMANN S, «Zehn Jahre kommunale Verwaltungsmodernisierung», in : JANN, W./BoGUMIL, J./ Bouckaert, G./BudÄus, D./HoltKamp, L./KißLer, L./KuhlmanN, S./MeZGer, E./Reichard, C./ Wollmann, H. (éd.), Status-Report Verwaltungsreform. Eine Zwischenbilanz nach zehn Jahren, Berlin, 2004

Breisig T., KÖNIG S., WengelowsKi P., Arbeitnehmer im Mitarbeitergespräch. Grundlagen und Tipps für den Erfolg, Frankfurt/Main, 2001

DRESCHER A., «Personalentwicklung im Reformprozess. Eine Standortbestimmung zur Personalentwicklung im öffentlichen Dienst», in : BECKER M., SchwARZ V. (éd.), Theorie und Praxis der Personalentwicklung. Aktuelle Beiträge aus Wissenschaft und Praxis, Mering, München, 2001, p. 54-70

Umfrage zur Personalentwicklung in der öffentlichen Verwaltung - Auswertung, Berlin (unveröffentlichtes Manuskript), 2005

FISCHER S., Human Resource Management und Arbeitsbeziehungen im Betrieb: eine empirische Untersuchung in der Chemischen Industrie Deutschlands, Mering, München 1998

Fischer S., Weitbrecht H., «Individualism and Collectivism: Two Dimensions of Human Resource Management and Industrial Relations. The Case of Germany», in : Industrielle Beziehungen, cahier 2-4, 1995

Goss D., Human Resource Management : The Basics, London u. a., 1997

GuEST David E., «Personnel Management: The End of Orthodoxy? », in : British Journal of Industrial Relations, cahier 29-2, 1991 p. 149-175 
JanN W., «Neues Steuerungsmodell», in : Blanke B., Bandemer S. von, Nullmeier F., Wewer G. (dir.), Handbuch zur Verwaltungsreform, 2ème édition, Opladen, 2001, p. 82-92

JESS A., Riggers M., "Verwaltungsreform und Gender Mainstreaming », in : BLANKE B., BANDEMER S. von, Nullmeier F., Wewer G. (éd.), Handbuch zur Verwaltungsreform, 2ème édition, Opladen, 2001, p. 187-195

KGSt [Kommunale Gemeinschaftsstelle für Verwaltungsvereinfachung], Das Mitarbeitergespräch. Rapport n¹3, Köln, 1992

Das neue Steuerungsmodell. Begründung-Konturen-Umsetzung. Rapp. n5, Köln, 1993

Personalentwicklung im Veränderungsprozess. Rapp. N³/2000, Köln

Das Mitarbeitergespräch in der Praxisbewährung. Rapp. N²/2002, Köln

KÖNIG K., Modernisierung von Staat und Verwaltung : Zum Neuen Öffentlichen Management, Baden-Baden, 1997

KÖNIG S., Human Resource Management, strategische Personalauswahl und Theorien industrieller Beziehungen. Eine Negotiated Order-Perspektive, Mering, Münick, 2005

KÖNIG S., REHLING M., «Wirkungsraster für mehr Transparenz in der Personalentwicklung öffentlicher Verwaltungen», in: KLuSMeyer, J., MeYerholt U., Wengelowski P.(éd.), Evaluation - Beratung Transfer, Oldenburg, 2005

KÖNIG S., REHLING M., Mitarbeitergespräche. Erfolgsfaktoren, Potenziale und Defizite in der öffentlichen Verwaltung, Düsseldorf, Edition der Hans-Böckler-Stiftung, vol. 156, 2006

LORSE J., «Personalentwicklung im öffentlichen Dienst - eine Zwischenbilanz», in : Verwaltungsrundschau, cahier 50-6, 2004, p. 194-198

Niedersächsisches Innenministerium, Geschäftsstelle für Verwaltungsreform (éd.), Die niedersächsische Landesregierung durch Personalentwicklung zukunftsfähiger gestalten. Das Rahmenkonzept der Personalentwicklung in Niedersachsen, Hannover, 1997a

Leitfaden: Das Mitarbeiter/Vorgesetzten-Gespräch (MVG) - ein Instrument der Personalentwicklung, Hannover, 1997b

Niedersächsisches Innenministerium (éd.), Auf dem Weg zu einem modernen Personalmanagement. Personalentwicklung in der niedersächsischen Landesverwaltung. Berichtsteil I (Bericht zur Umsetzung des Kabinetts-Beschlusses vom 18.02.1997 und Handlungsorientierungen für die weitere Entwicklung) und Berichtsteil II (Einzelergebnisse der Evaluation Personalentwicklung 1997 bis 1999), Hannover, 2002

Oechsler Walter A., Personal und Arbeit: Grundlagen des Human Resource Management und der Arbeitgeber-Arbeitnehmer-Beziehungen, 7ème édition, München ; Wien, 2000

REHLING M., Pilothafte Erprobung von Personalentwicklung an einer Hochschule: Ein strukturationstheoretisches und mikropolitisches Erklärungsmodell (Dissertation in Vorbereitung), 2006

ReICHARD C., «Personalmanagement», in : Blanke B., BANDEMER S. von., Nullmeier F., WeWER G. (éd.), Handbuch zur Verwaltungsreform, 2ème édition, Opladen, 2001, p. 180-186

REICHARD C., «Verwaltungsmodernisierung in den Bundesländern», in : JANN W., BOGUMIL J., BOUCKAERT G., Budäus D., Holtkamp L., Kißler L., Kuhlmann, S., Mezger E., Reichard C., Wollmann H., (éd.), Status-Report Verwaltungsreform. Eine Zwischenbilanz nach zehn Jahren, Berlin, 2004, p. 87-99

SCHREYÖGG G., OECHSLER Walter A., WÄCHTER H., Managing in a European Context : Human Resources, Corporate Culture, Industrial Relations. Texts and Cases, Wiesbaden, 1995

STAEHLE Wolfgang H., «Human Resource Management (HRM) », in : ZfB, cahier 58-5/6, 1988, p. 576-587

Management - eine verhaltenswissenschaftliche Perspektive, 6ème édition, München, 1991

STOREY J. : Developments in the Management of Human Resources. An Analytical Review, Oxford, 1992

Tichy Noel M., Fombrun Charles J., DeVAnNA M.A., «Strategic Human Resource Management», in : Sloan Management Review, 1982, H. 23-2, p. 47-61

TONDORF K., BAHNMÜLleR R., Klages H., Steuerung durch Zielvereinbarungen : Anwendungspraxis, Probleme, Gestaltungsüberlegungen, Berlin, 2002

TONDORF K., JOCHMANN-DÖLL A.,), «Monetäre Leistungsanreize im öffentlichen Sektor», in : WSI Mitteilungen, cahier 57-8, 2004, p. 428-434 



\title{
Deutsche Telekom AG: vers un management rénové des ressources humaines
}

\author{
Dietmar FRINGS
}

Les transformations économiques qu'ont connues les secteurs dérégulés ne sont pas sans conséquences sur les pratiques sociales des entreprises. La façon dont sont considérées les ressources humaines dans la stratégie de l'entreprise joue ici un rôle crucial: ressource stratégique ou variable d'ajustement? L'exemple de la Deutsche Telekom AG présentée ici par son responsable des relations internationales du travail est à cet égard éloquent : entreprise de services dans un secteur technologique hautement concurrentiel, elle s'efforce depuis dix ans d'élaborer une politique de gestion des ressources humaines spécifique qui, en s'appuyant sur les partenaires sociaux, contribue à surmonter les conséquences sociales des mutations du secteur tout en s'engageant sur la voie de l'innovation sociale.

La Deutsche Telekom a fêté en 2005 son dixième anniversaire. Nous ne nous considérons donc plus comme une entreprise publique de services mais comme une entreprise privée ayant ses origines dans la Poste allemande, dont l'histoire s'étend sur plus de 500 années. La Deutsche Telekom AG, par la voix de la Direction de Ressources humaines mais aussi par la voix du management de l'entreprise et en particulier du président du Directoire, a toujours souligné que le personnel constitue le principal capital de l'entreprise

Si l'on veut avancer avec les collaborateurs, il faut aujourd'hui se doter d'un nouveau concept directeur. Pour la Deutsche Telekom, cela signifie que ce concept directeur ne doit pas être décrété d'en haut, mais être élaboré dans le cadre d'un dialogue mené avec les personnels. C'est ce que nous avons entrepris en 2004 afin de donner une nouvelle orientation au groupe et un point d'appui aux collaborateurs en leur transmettant les valeurs de l'entreprise.

L'idée du groupe Deutsche Telekom est de s'engager, en sa qualité d'entreprise de services leader dans le secteur des télécommunications et des technologies de l'information, pour offrir un meilleur avenir à la société, en proposant un niveau optimal d'efficacité, de qualité et d'innovation, au bénéfice du client. En interne, six valeurs propres à l'entreprise doivent permettre d'atteindre cet objectif. Il faut ainsi augmenter la valeur de l'entreprise, pour les collaborateurs au même titre que pour les actionnaires : il est essentiel de maintenir, voire d'augmenter la valeur d'une entreprise, d'une part afin de garantir à l'actionnaire un revenu satisfaisant sur le capital investi, d'autre part pour être en mesure de proposer ultérieurement aux collaborateurs des emplois stables.

Etre «partenaire du client » constitue la seconde valeur. Nous ne fournissons pas de services publics : nous avons des clients. Nous n'avons donc plus comme auparavant des abonnés déposant une demande afin d'obtenir, après un certain temps, une ligne 
téléphonique. Aujourd'hui, nous nous devons de satisfaire dans les meilleurs délais et avec un haut niveau de qualité les souhaits du client, si nous voulons nous maintenir dans la compétition.

Une entreprise technologique vit de l'innovation. Nous voulons créer pour nos collaborateurs un climat favorable au développement, où l'on puisse proposer de nouvelles idées, même si elles n'aboutissent pas en l'état et doivent être retravaillées.

Autre valeur, le respect signifie que nous devons exploiter notre diversité culturelle et nous soutenir mutuellement. L'innovation représente la troisième valeur, tandis que l'intégrité garantit un mode de communication ouvert et sincère : nous tenons nos engagements. Enfin, la valeur «Top excellence » : elle signifie que des prestations de qualité doivent être rémunérées en conséquence et qu'il convient donc de se doter de systèmes idoines.

Figure 1 : Evolution des valeurs

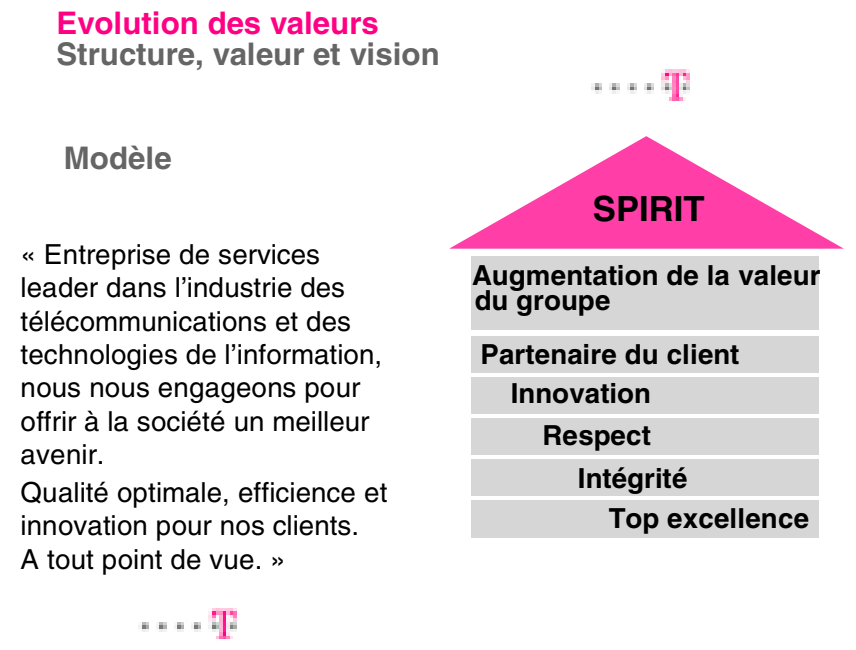

Source : Deutsche Telekom AG

Nous développerons différents points dans les paragraphes suivants. Nous proposerons un rappel de la privatisation, des conséquences sur l'emploi et de l'accompagnement de cette mutation par les partenaires sociaux. Nous poserons alors la question suivante : comment le personnel, partenaire stratégique du management, peut-il contribuer à la mise en oeuvre du changement, c'est-à-dire ne pas seulement réagir mais aussi agir ? Quelles évolutions sont en jeu, en termes de personnel et de valeurs ? Quels défis l'avenir réserve-t-il ? Les changements ne font en effet que commencer.

D'où venons-nous? Notre passé est celui d'une administration publique et nous sommes maintenant depuis dix ans une société anonyme. Au cours de ces dix années, le changement a été permanent. Depuis 2000, l'entreprise est structurée en quatre grands secteurs qui se concentrent chacun sur leurs clients respectifs : T-Mobile pour les clients de la téléphonie mobile, T-Online pour l'internet, T-Systems pour la clientèle professionnelle et T-Com qui s'adresse à la clientèle privée et a donc en charge le réseau à large bande. 
Figure 2: Retour sur les mutations - du prestataire national à l'acteur global

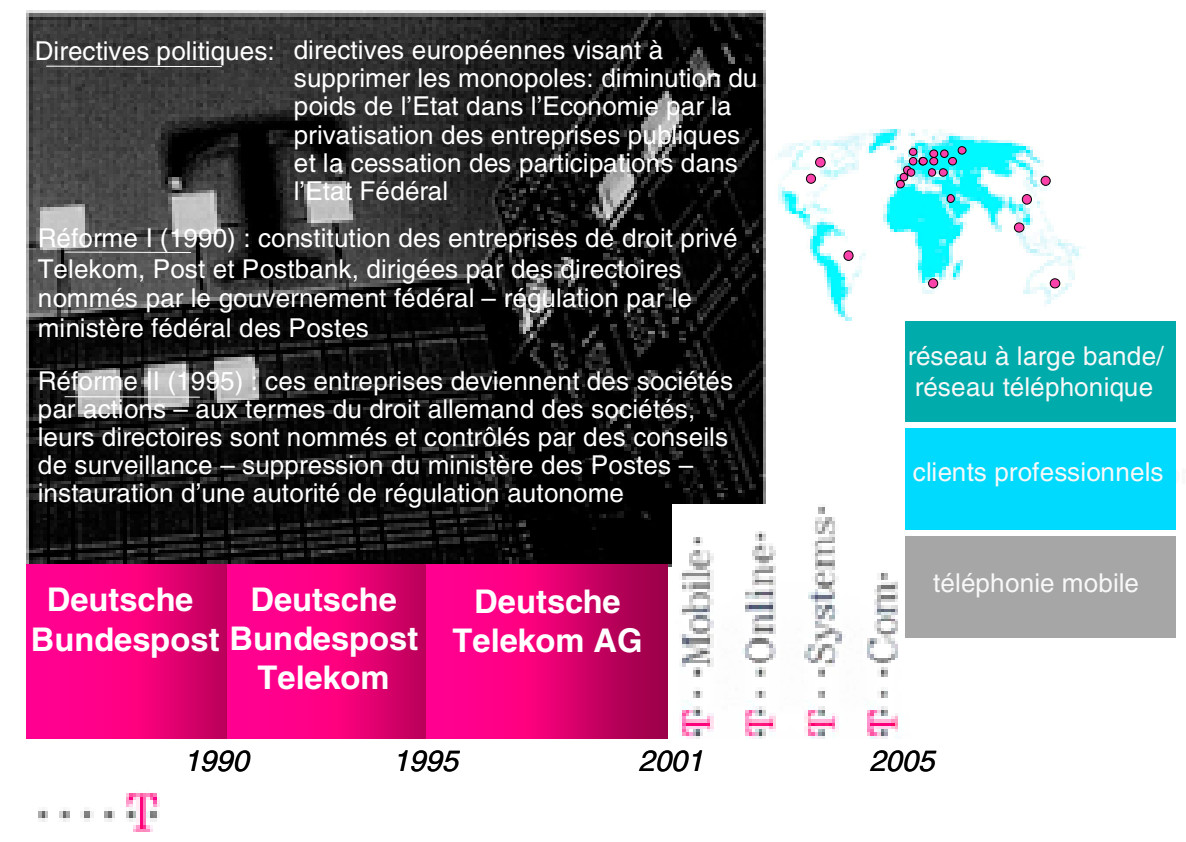

Source: Deutsche Telekom AG

Le marché et la libéralisation ont entraîné un profond bouleversement plaçant l'entreprise face à des enjeux de taille. On compte dorénavant en Allemagne 2300 prestataires de services téléphoniques. Les tarifs ont fortement diminué. Certains prix à la minute ne sont plus qu'à $3 \%$ du niveau antérieur. Ces bouleversements n'ont évidemment pas épargné le personnel et les effectifs et d'importants efforts doivent être déployés pour développer de nouveaux domaines d'activité pour le groupe Deutsche Telekom et ses collaborateurs. Mais nous affrontons cette compétition et voulons stimuler l'innovation en Allemagne. Au cours des dix dernières années, le groupe a investi 25 milliards d'euros dans les nouveaux Länder, puisqu'il a fallu reconstruire entièrement le réseau dès 1990, au lendemain de l'unification. Les standards valables sous le régime socialiste n'étaient ni adaptés à l'activité économique allemande, ni en mesure de soutenir le développement des Länder orientaux. Les télécommunications constituent l'épine dorsale de l'activité économique. Sur le plan macroéconomique, le groupe Deutsche Telekom a dès lors payé le prix fort pour élever les télécommunications dans l'Est de l'Allemagne au plus haut niveau.

Nous poursuivons ces efforts, la tendance actuelle étant de développer le réseau à large bande pour l'internet. Désormais, le client veut être partout joignable grâce aux réseaux mobiles et nous devons donc répondre à cette exigence. Nous avons multiplié au cours des dernières années les lieux permettant de se raccorder directement au réseau avec un ordinateur portable (hôtels, gares, aéroports, ....). 
Du point de vue de l'emploi, la Deutsche Telekom était en 1994 une entreprise nationale, focalisant son activité sur l'Allemagne et employant environ 230000 salariés. Aujourd'hui, les effectifs du groupe s'élèvent à 250000 , ce qui ne signifie nullement que nous ayons augmenté le nombre de collaborateurs en Allemagne. 110000 emplois y ont au contraire été supprimés. Chaque année, 10000 collaborateurs ont quitté l'entreprise ; par ailleurs, nous avons transféré 27000 agents du siège vers des filiales, avec des programmes de qualification. L'émergence de nouveaux secteurs d'activité, par exemple dans les nouvelles technologies ou le marché du mobile, qui a littéralement explosé au cours des dix dernières années, a donné naissance à de nouveaux emplois que nous avons pu pourvoir en puisant dans les effectifs en place, c'est-à-dire les agents du réseau téléphonique.

Le niveau actuel de nos effectifs résulte des nouvelles acquisitions à l'étranger. En clair, le groupe Deutsche Telekom s'est internationalisé en dix ans et a gagné 80000 nouveaux collaborateurs dans le monde entier. Pour l'heure, c'est aux Etats-Unis que les effectifs ont le plus augmenté pour s'établir à près de 20000 salariés de T-Mobile US.

Figure. 3: Variation des effectifs au sein du groupe

\section{Des réductions d'effectifs socialement acceptables}

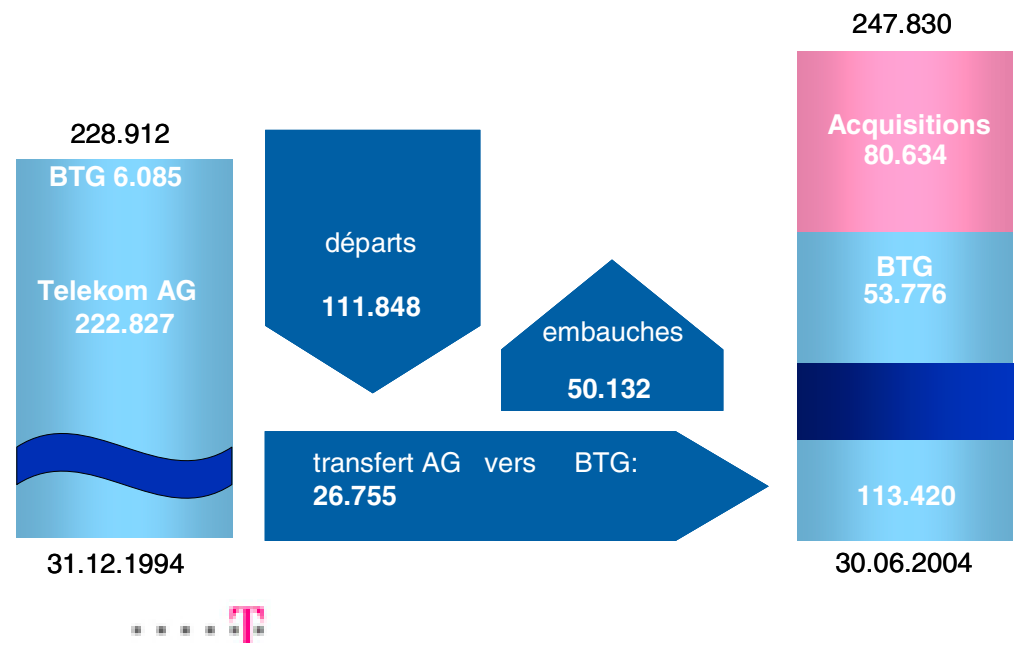

Source : Deutsche Telekom AG

Nous conservons un héritage historique spécifique dans notre effectif, ce dernier étant marqué par des structures d'emploi issues de la fonction publique. En ce qui concerne les rémunérations ou les garanties d'emploi, il a été quasiment impossible de modifier ces structures de telle sorte que nous puissions effectivement congédier un agent : en Allemagne, $50 \%$ de nos collaborateurs sont fonctionnaires. Il s'agit d'agents disposant donc d'une situation administrative spécifique et permanente vis-à-vis de l'Etat mais qui travaillent au sein de la Deutsche Telekom.

Par ailleurs $25 \%$ des agents bénéficient d'une protection permanente contre les licenciements, équivalente à celle des fonctionnaires. Et pour encore $24 \%$ des salariés 
actuellement, nous avons négocié avec les syndicats le non recours aux licenciements jusqu'à fin 2008. Cela signifie que ces collaborateurs ne se retrouveront pas sur le marché du travail en cas de mesures de suppression de postes.

Au cours des dix années précédentes, Deutsche Telekom n'a procédé à aucun licenciement. Nous avons financé des programmes de suppression de postes, nous avons versé des indemnités financières, présenté des programmes de retraite anticipée pour un montant total dépassant les 4 milliards d'euros. La suppression de 110000 postes en 10 ans dont il a été question précédemment est quasiment passée inaperçue dans l'opinion publique. Il s'agit là d'une réduction d'effectifs socialement très acceptable, accompagnée par les partenaires sociaux, mais qui a néanmoins représenté une charge financière considérable pour Deutsche Telekom.

Figure 4: Restrictions relatives aux suppressions de poste chez DTAG Part des fonctionnaires dans les effectifs de la Deutsche Telekom : différences régionales

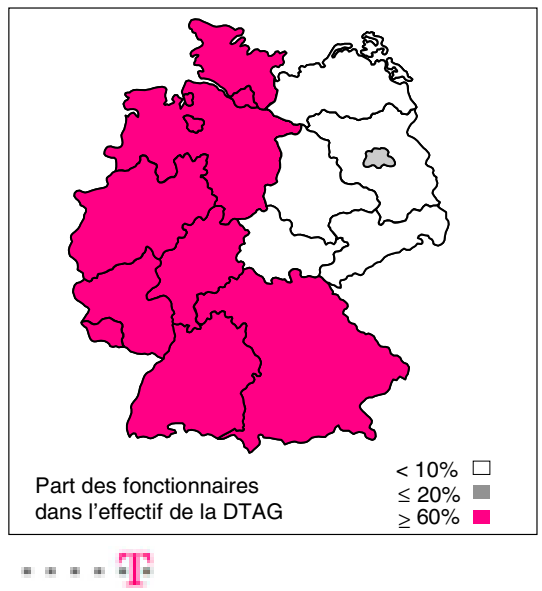

- Imbroglio entre nécessité régionale/ fonctionnelle et possibilités juridiques/effectives

- Le licenciement de 30000 salariés provoquerait par ailleurs:

-une hausse du ratio de fonctionnaires à $65 \%$. -une nouvelle dégradation de la structure d'âge puisque les licenciements porteraient tendanciellement sur les salariés les plus jeunes

Source: Deutsche Telekom AG

Le graphique précédent montre où surgissent les problèmes en cas de rationalisation ou de simplification des processus. Dans la partie occidentale du pays, le groupe emploie majoritairement des fonctionnaires, tandis qu'il emploie majoritairement des salariés dans la partie orientale. Autrement dit, les collaborateurs employés à l'Ouest bénéficient d'un niveau élevé de protection de leur emploi, contrairement à leurs collègues des nouveaux Länder. Si nous devions agir comme le ferait une entreprise normale, il nous faudrait donc licencier des collaborateurs dans les Länder de l'Est et transférer des effectifs des anciens vers les nouveaux Länder. On imagine aisément ce que cette politique aurait d'explosif sur le plan social, ce que nous refusons.

Deutsche Telekom assume par ailleurs des responsabilités sociales considérables. Nous-mêmes avons un besoin quasi nul de nouveaux collaborateurs puisque nous avons supprimé des postes en 2004 et allons nous séparer de 30000 collaborateurs environ durant les prochaines années. Malgré cela, nous formons chaque année 4000 apprentis et investissons 220 millions d'euros par an dans l'apprentissage. Sur ces 4000 
apprentis, seuls 400 d'entre eux intégreront le groupe, en l'absence de besoin. On voit là que la Deutsche Telekom assume une responsabilité sociale sensiblement plus importante en matière d'apprentissage que bien d'autres entreprises comparables, qui ne sont pourtant pas confrontées à ce problème majeur de réduction des effectifs.

Nous avons conclu en 2004 un pacte pour l'emploi pour le groupe Deutsche Telekom. Nous étions alors face à un défi : prolonger l'accord de protection contre les licenciements, qui expirait fin 2004, mais seulement si de nouvelles activités étaient créées. Car comme nous l'avons dit précédemment, nous enregistrons un excédent de 30000 employés qui normalement auraient dû être rendus au marché du travail. Nous avons donc négocié avec les instances syndicales la création d'environ 10000 nouveaux emplois par une réduction collective du temps de travail hebdomadaire. La durée hebdomadaire de travail est passée de 38 heures pour l'ensemble du groupe Telekom à 34 heures avec pour effet la création de 10000 emplois. Cette mesure ne suffit évidemment pas à remettre dans l'emploi les 30000 personnes que nous ne pouvons plus employer dans le domaine traditionnel des télécommunications. Pour un tiers, le problème a été résolu grâce à la réduction du temps de travail. Nous avons créé notre propre agence de service à la personne, qui s'occupe de remettre en emploi les collaborateurs ayant perdu leur travail. Nous avons créé de nouvelles entreprises, subventionnées naturellement, qui reçoivent des contrats et emploient des collaborateurs. A titre d'exemple, nous disposons d'une unité employant près de 3000 salariés dans le domaine des centres d'appel, ou encore d'une unité, en cours de constitution, qui comptera à terme 5000 salariés environ pour des tâches de montage.

Nous avons mis en oeuvre d'autres projets destinés à générer de l'emploi. Le troisième pilier de cette agence pour l'emploi est le placement individuel sur le marché externe du travail. Par le biais de cette initiative pour garantir l'emploi, nous voulons somme toute accompagner les collaborateurs quittant groupe Deutsche Telekom vers un nouvel emploi, et non les abandonner au chômage.

Ce modèle coûte au groupe un milliard d'euros par an. Nous ne sommes pas une entreprise publique. $43 \%$ de notre capital est, il est vrai, toujours détenu par l'Etat allemand, mais nous avons aussi des actionnaires extérieurs dans le monde entier. Les actionnaires aux Etats-Unis ou au Japon nous interrogent naturellement sur les motifs de nos dépenses et nous devons donc envisager une réduction de ces dépenses dans les prochaines années.

En résumé, on peut donc affirmer que la mutation du groupe n'a pas eu pour conséquence un afflux de chômeurs pesant sur le marché du travail allemand. Nous n'avons pas eu recours à des subventions de l'Etat allemand et avons, lorsque cela s'avérait possible, transféré des emplois dans des régions économiquement fragiles, à l'instar des call-centers. Enfin, nous avons renoncé aux licenciements économiques. Nous avons par ailleurs notre propre unité chargée du placement des collaborateurs privés d'emploi. Toutes ces mesures ont été largement prises en accord avec les partenaires sociaux.

Qui sont donc les partenaires sociaux de la Deutsche Telekom ? Nous avons d'une part naturellement des partenaires avec lesquels nous menons des négociations contractuelles : il s'agit en Allemagne du syndicat des services Ver.di (Vereinigte Dienstleistungsgewerkschaft), le plus grand syndicat allemand avec son département sectoriel Télécommunications. D'autre part, nos partenaires sont les conseils d'établissement au sein de la Deutsche Telekom. Le fait que les conventions collectives soient négociées directement avec l'entreprise est en réalité un cas assez peu typique en Allemagne, mais 
qui s'explique par la tradition de Deutsche Telekom : nous négocions nous-mêmes nos accords collectifs, alors que généralement en Allemagne, ce sont les fédérations patronales, donc des regroupements d'employeurs, qui négocient avec les syndicats de branche. Nous sommes nous-mêmes directement impliqués et négocions accords collectifs et rémunérations au sein de notre unité pour le compte de 20 entreprises, comme le ferait une association interne d'employeurs. Le modèle allemand de cogestion prévoit la participation des représentants des salariés aux décisions de l'entreprise, au niveau du directoire et du conseil de surveillance. Le conseil de surveillance est un organe de contrôle qui veille sur le travail du directoire, mais dans des limites strictement définies. A cet organe de contrôle participent à la fois des délégués du personnel et des représentants syndicaux. Pour citer un exemple, le responsable des négociations collectives au sein de Ver.di est également vice-président du conseil de surveillance de la Deutsche Telekom. C'est évidemment un exercice périlleux dans la pratique, puisque ce modèle repose fortement sur des personnes et sur leur intégrité. D'un côté, les syndicats ont une connaissance très exacte de l'entreprise ; mais de l'autre, il faut naturellement élaborer des positions dans le cadre des négociations collectives, afin d'amener les partenaires à des concessions. Il faut pourtant souligner que la Deutsche Telekom s'est toujours préoccupée d'entretenir de bonnes relations avec les partenaires sociaux, et qu'elle y reste très attachée. Un dernier point, assez inédit en Allemagne : nous prélevons pour le syndicat les cotisations de ses membres directement sur le salaire.

Quelle a été la mission de la direction des ressources humaines dans ce processus de changement? Dans bien des entreprises, la DRH est un prestataire de services: opérations d'écriture, établissement des contrats. Nous ne nous sommes jamais reconnu dans ce rôle: la mission de la DRH doit toujours être l'accompagnement actif des processus et des évolutions. D'où notre initiative stratégique.

Figure 6: Systématique de la stratégie des ressources humaines du groupe Le management comme partenaire stratégique

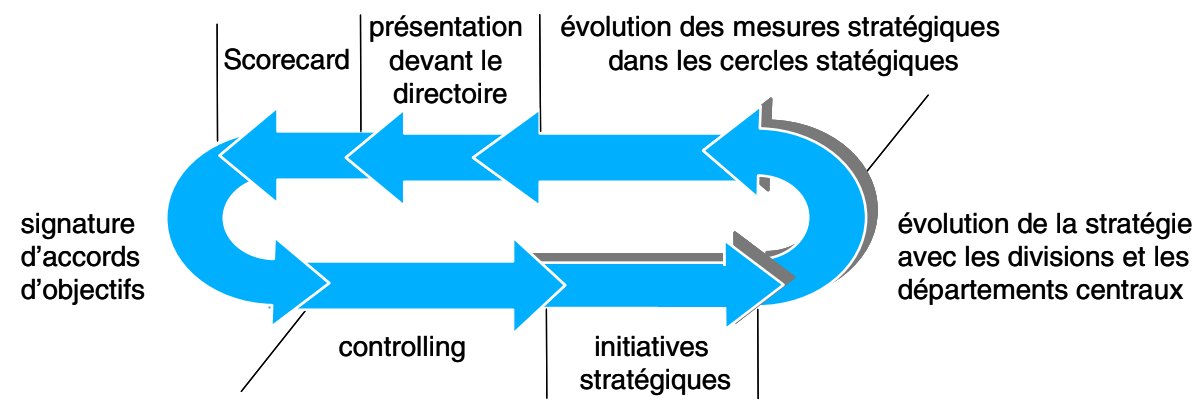

Source : Deutsche Telekom AG

Chaque année, nous étudions quelle sera la stratégie du groupe l'année suivante et analysons, au niveau du groupe et au niveau des divisions, ce que nous devons faire en matière de ressources humaines pour renforcer la stratégie du groupe. Ces mesures sont élaborées au sein de groupes de travail, puis présentées au directoire ; nous définissons 
également des indicateurs, c'est-à-dire des chiffres vérifiables, intégrés dans chacun des accords d'objectifs conclus avec l'encadrement, ceci afin d'être en mesure d'évaluer des objectifs individuels. Le contrôle de gestion mené en fin d'année implique que le contenu de la stratégie est à son tour évalué afin que nous puissions voir si nous avons atteint nos objectifs.

Figure 7: Mission et objectifs de la stratégie RH du groupe

\section{La compétence, l'engagement et le professionalisme des collaborateurs sont les principaux facteurs de succès}

En tant que business partner, nous effectuons une gestion des RH active et respectueuse des intérêts du client, dont la contribution à l'augmentation de la valeur de l'entreprise peut être mesurée.

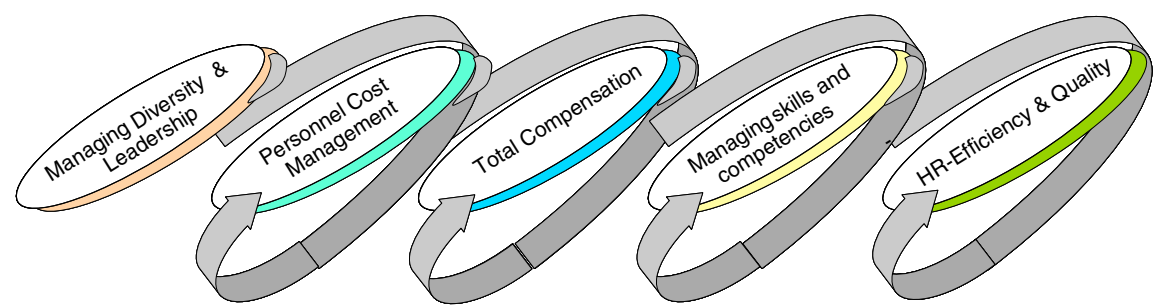

- Introduire la notion Manager les coûts

- Augmenter

l'engagement du personnel dans une logique de productivité

des collaborateurs - Gérer les effectifs

- Promouvoir l'idée de au niveau du groupe performance sociale $\bullet$ Promouvoir

- Améliorer l'image de l'employabilité et l'employeur

le placement hors du Groupe pour des missions externes

- Optimisation - Recrutement en du système de interne des cadres rémunération et des primes

- Harmonisation

des conditions d'emplois des fonctionnaires et salariés sur le long terme

- Développer l'idée pétences en terme de service
- Diminution des coûts de gestion RH

- Augmentation de la des salariés envisagé qualité du service $\mathrm{RH}$ de client et les com-

$$
* .+.72
$$

Source: Deutsche Telekom AG

En notre qualité de manager des ressources humaines, nous avons tout d'abord envisagé les cinq grands points sur lesquels Deutsche Telekom veut se concentrer prioritairement. Nous devons d'abord mettre en avant différents facteurs : les collaborateurs, l'engagement (commitment) des personnels, la performance sociale (Social Performance). En d'autres termes, il nous faut expliquer clairement aux collaborateurs les valeurs de l'entreprise, les faire vivre et faire en sorte que les cadres s'attachent sincèrement à les transmettre. La gestion des coûts du personnel, c'est-à-dire la problématique coûts/ productivité, constitue un point très important. La concurrence nous contraint à nous interroger sans cesse sur les moyens de réaliser des économies sur les coûts de personnel sans procéder à des licenciements. Troisième point : il faut continuer d'aménager le système des rémunérations. Pour être un employeur attractif, il nous faut garantir l'attractivité du système de rémunération. Le quatrième point concerne le 
management de la formation et du développement du personnel. Une entreprise en mutation doit justement accorder une grande importance au développement du personnel sous peine de se priver d'un know how renouvelé. La diminution des embauches contraint à mettre les collaborateurs en situation de miser sur les nouvelles techniques et l'innovation. Un management effectif des RH va donc de soi.

Figure. 8: Evolution des valeurs - Commitment

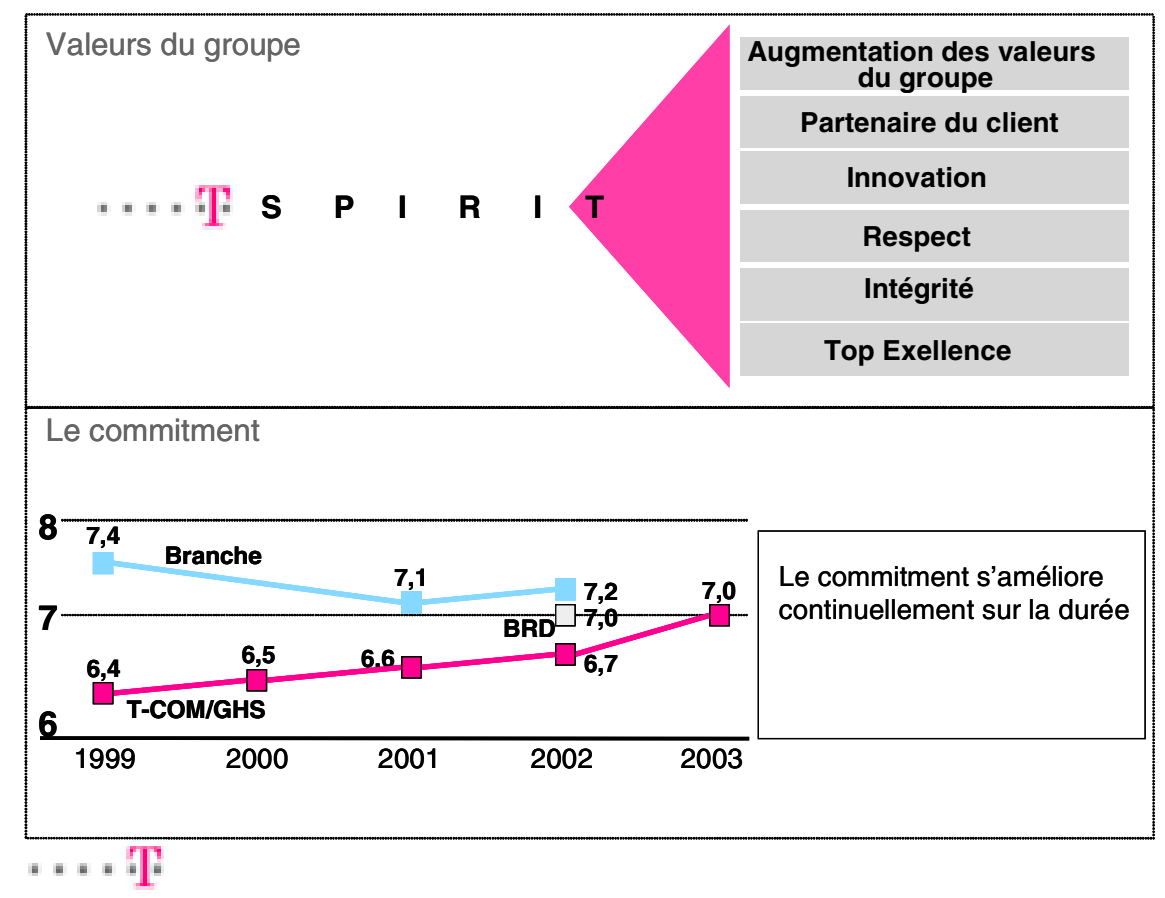

Source: Deutsche Telekom $A G$

La mesure de «l'engagement» (commitment) montre-t-elle que nous sommes sur la bonne voie ? Evalué chaque année par une enquête auprès des collaborateurs, cet indicateur nous permet de connaître leur opinion de l'entreprise. Au cours de ces dernières années de changement, nous avons enregistré une hausse du commitment, c'est à dire de l'engagement des collaborateurs envers la Deutsche Telekom, en dépit des nombreuses ruptures qui ont été exigées d'eux. Nous considérons donc que nous sommes sur une excellente voie, ce qui ne signifie nullement qu'à l'avenir, nous pourrons nous reposer sur nos acquis. La concurrence montre que l'efficacité, les nouveaux produits, et les innovations devront nous aider à développer de nouveaux domaines d'activité, et donc de nouveaux emplois. Dans le domaine des communications de proximité, notre part de marché est actuellement encore de deux tiers. En un an, la concurrence nous a déjà privés d'une part importante de ce marché et dans le domaine de la téléphonie mobile, le moteur de l'emploi pour la Deutsche Telekom, nous enregistrons aujourd'hui une saturation du marché, $90 \%$ de la population utilisant aujourd'hui un portable. 
Figure 9: Modifications de la structure du marché et de la concurrence

\section{Concurrence plus agressive sur le segment de la bande large. Couplage téléphonie mobile et réseau. Renforcement des parts de marchés des compétiteurs.}

\section{Observation actuelle du marché}

-Une concurrence
plus agressive
sur le segment
de la bande large.
Ex: Hansenet/
Telecom Italia
Couplage
téléphonie mobile/
réseau.
Ex: coopération
entre Freenet
et E-Plus

- Développement des parts de marché des compétiteurs dans le nouveau domaine des connexions à bande large
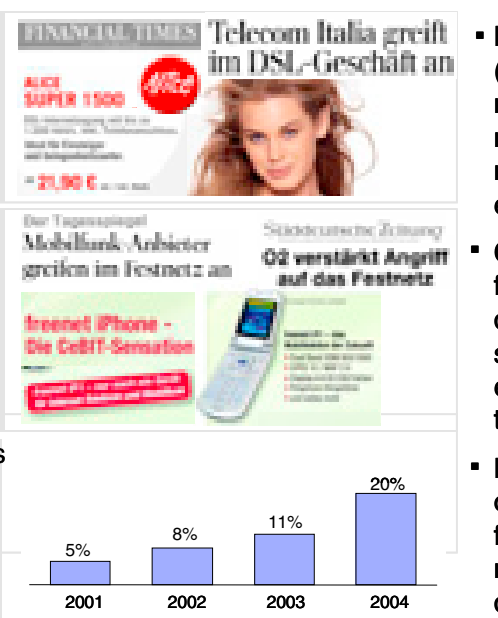

- Les nouvelles technologies (VolP, internet mobile) modifient l'évolution des marchés de la téléphonie mobile, du réseau et de l'interne

- Certains produits et services de fournisseurs de télécommunications, de software ou de hardware se recoupent davantage et suppriment les frontières traditionnelles

- Les cessions et offres de coopération font émerger sur des marchés traditionnels de nouveaux acteurs (Tschibo par exemple)

Source: Deutsche Telekom AG

Le renforcement de la concurrence vient de l'étranger: Certains grands fournisseurs attaquent la Deutsche Telekom dans les zones urbaines par une politique d'offres promotionnelles, comme le fait Telecom Italia, ou en proposant des bouquets de prestations (réseau + téléphonie mobile). Cette situation de concurrence assumée en Allemagne nous contraint donc à toujours convaincre par de nouveaux produits et de nouvelles idées.

Si l'on se réfère à la figure 10, l'avenir semble évident. Nous comptions, il y a 20 ans, encore quelque 6300 relais locaux, hébergeant des installations techniques dont la maintenance était assurée par des techniciens. Aujourd'hui, nous n'avons plus que 2500 relais digitaux pourvoyeurs d'emplois sur place. Mais on sait déjà que moins de 10 centres de calcul suffiront dans quinze ans à piloter l'ensemble du réseau téléphonique allemand, les réseaux de téléphonie mobile et la clientèle professionnelle. En termes d'emplois et pour parler clairement, nous ne savons pas encore comment les choses vont évoluer. Nous avons en outre un déficit de productivité, la Deutsche Telekom ayant toujours été une entreprise proposant un haut niveau de rémunération. Si l'on prend l'exemple des centres d'appel, les salaires sont ainsi le double de ceux proposés par nos concurrents. Dans un contexte concurrentiel, nous devons réduire nos coûts élevés de personnel si nous voulons continuer d'exister, ce qui signifie que les partenaires sociaux 
et les collaborateurs vont devoir accepter des coupes dans les systèmes de protection et de rémunération, afin de garantir les emplois sur le long terme.

\section{Figure 10: Nouvelles technologies}

\section{Renforcement de l'automatisation dans les domaines du Marketing et de la vente. Concentration technique et effets sur l'emploi}

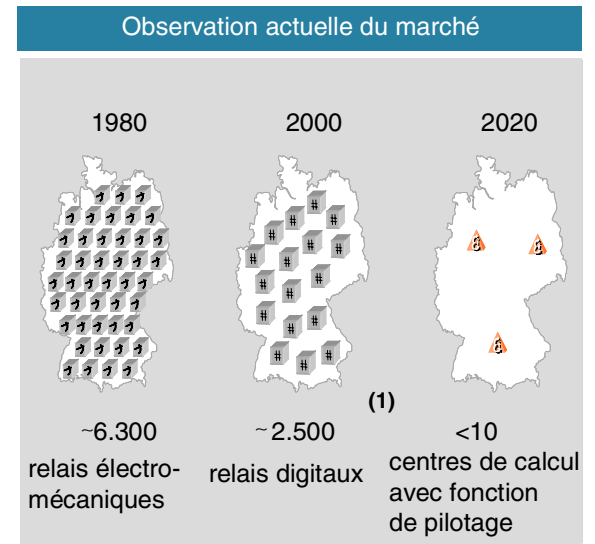

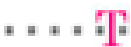

Les nouvelles technologies induisent des effets en termes d'emploi pour la Deutsche Telekom à différents stades de la chaîne de création de valeur.
- Marketing/vente : automatisation des processus de GRC (CRM-Customer Relationship Management/gestion de la relation client), substitution des canaux de vente traditionnels par le concept de eChannel, automatisation des procédures de commandes
- Production/technique : concentration technique au sein de centres de calcul assumant des fonctions de pilotages

Source : Deutsche Telekom AG

DÈS LORS, mon message tient en quatre points:

- Le management des ressources humaines est un facteur essentiel pour le succès d'une privatisation. Il faut se confronter à certains défis, en particulier dans une situation de concurrence.

- En raison du poids des dépenses de personnel, il faut augmenter impérativement la productivité.

- Nous tentons de placer des collaborateurs sur de nouvelles missions.

- Les valeurs et la culture de l'entreprise ne doivent pas être négligées. Il faut en outre proposer des systèmes de rémunération et d'emploi axés sur les performances, ainsi que des systèmes de développement des compétences des personnels. On ne doit pas se focaliser exclusivement sur les suppressions de postes mais aussi investir dans les collaborateurs, c'est à dire dans leur employabilité. 

- III -

RELATIONS SOCIALES

ET REPRÉSENTATION DU PERSONNEL 



\title{
Evolution des relations sociales dans les services d'intérêt général : l'exemple de la RATP
}

\author{
Philippe MONCOURRIER
}

La mutation profonde du cadre économique prévalant pour les services d'intérêt général, marqué depuis les années 90 par des logiques de libéralisation et de dérégulation, n'est pas sans conséquences sur les modèles organisationnels et les pratiques sociales des entreprises évoluant dans ce secteur. La complexification du contexte économique et social, mais aussi l'impasse en termes d'efficacité, d'équité et de capacité d'adaptation aux besoins exprimés par la population, à laquelle avaient abouti certaines pratiques de gestion publique et de relations sociales, ont rendu inéluctable l'évolution des organisations et des cultures de travail. De nouveaux modes de management sont adoptés qui laissent apparaître des phénomènes d'hybridation intéressants entre spécificités des contraintes de la gestion publique et logique de marché. Cette contribution apporte le témoignage d'un observateur et acteur privilégié sur la capacité de transformation au cours des années 90 de la RATP, grande entreprise française de transport public, d'une structure sclérosée en une entreprise qui peut aujourd'hui prétendre anticiper les évolutions futures, dans une relation triangulaire prenant en compte les besoins de l'usager-client.

L'histoire de la RATP est assez singulière au regard des évolutions actuelles dans le secteur d'activité des services publics en France. La RATP a eu beaucoup de chance parce qu'elle a failli mourir il y a quinze ans environ. Et c'est grâce à ce risque énorme que l'entreprise a finalement réussi à se reconstituer et à aborder la période d'aujourd'hui d'une manière assez différente et avec suffisamment d'antériorité dans de nouvelles pratiques pour que l'on ait atteint un niveau de confiance relativement élevé par rapport à la mise en concurrence, à l'ouverture des marchés, et la fin du monopole annoncé, qui sera effectif d'ici quelques années.

\section{Une évolution en décalage avec la demande des usagers}

Un rappel historique s'avère nécessaire pour comprendre ce qui s'est passé. La RATP a cinquante ans d'histoire. Elle est née après la guerre dans l'effort de reconstruction nationale avec la volonté des pouvoirs publics de constituer sur le noyau de l'actuelle Ilede-France, à savoir Paris et sa proche banlieue, une structure de transports en commun suffisamment performante, avec un maximum de continuité dans son activité pour acheminer la main d'œuvre. ${ }^{1}$ A cette époque déjà, cette région constituait un poumon

\footnotetext{
${ }^{1}$ Ce thème revient aujourd'hui avec la problématique du service minimum.
} 
économique important pour la France. La RATP est une entreprise contrôlée à $100 \%$ par l'Etat avec un statut d'EPIC. ${ }^{2}$

La RATP était en péril pour plusieurs raisons, qui tenaient principalement à la structure de l'entreprise, aux modalités managériales et à la forme de ses relations sociales. Une dynamique s'était installée au fil des décennies qui avait généré une incapacité à répondre aux demandes de ceux qu'on n'appelait pas encore les 'clients'. L'entreprise, de sa création jusqu'en 1990, était structurée de manière classique, centralisée et pyramidale, avec des décisions prises presque exclusivement au sommet. Une multitude de niveaux et d'appellations hiérarchiques conduisait à une impossibilité à exercer une quelconque responsabilité locale réelle. Cette situation a fini par créer des difficultés, y compris d'ordre psychologique, parmi le personnel encadrant. Dans le même temps, ce mode de fonctionnement était couplé à une culture syndicale fortement politisée avec la CGT comme syndicat majoritaire qui cultivait un lien très étroit avec le parti communiste. De ce contexte est né un mode d'échanges entre partenaires sociaux basé sur le conflit et l'opposition et sur une pratique très limitée de la négociation collective. Ce mode de fonctionnement a prévalu jusque dans les années 70. Cette situation aurait d'ailleurs pu perdurer si plusieurs facteurs convergents n'avaient conduit à un résultat sans appel: les clients ne suivaient plus. La fin des années 60 / début des années 70 correspond en effet à l'éclosion en France des villes nouvelles et d'une urbanisation étendue dans l'espace, qui a généré un recours disproportionné à la voiture particulière. Paradoxalement, la RATP a contribué à la création de ces villes nouvelles et zones périphériques par l'installation et le développement du RER, trains de moyenne capacité exploités en coopération avec la SNCF et qui permettent précisément d'irriguer en partie ces populations, sans toutefois couvrir l'ensemble de leurs déplacements. On a donc assisté à un accroissement spectaculaire de l'utilisation de la voiture individuelle au détriment des transports en commun, qui s'est ajouté aux mutations culturelles que l'on a connues un peu partout, mai 68 étant le symptôme significatif de ces évolutions, avec le refus de la triade "métro, boulot, dodo », la RATP incarnant là le métro. Le métro a alors commencé à être moins utilisé par des populations dont le comportement évoluait avec une nouvelle approche de leurs déplacements, et le choix d'autres moyens de transports répondant à une envie de vivre autrement (plus de loisirs dans le quotidien, déplacements différenciés).

\section{L'organisation interne dans l'impasse}

Cette situation a obligé l'entreprise à s'adapter à cette nouvelle demande, alors même que sa production était structurée en fonction de demandes simples (heure de pointe du matin, heure de pointe du soir, et entre ces deux moments, moyenne assez stable). Du coup, le mode d'organisation convenait assez bien à ce schéma assez immuable, dès lors que l'entreprise était incapable de s'adapter. Mais ces dysfonctionnements ont progressivement grippé le système, tandis que venaient s'ajouter des difficultés particulières à la France et notamment à la RATP : l'alternance politique de 1981 a en effet créé une situation exceptionnelle pour la RATP qui est alors devenue la seule entreprise publique à être dirigée par un dirigeant communiste, lui-même sous tutelle d'un des quatre ministres communistes du gouvernement. Compte tenu de l'antériorité des équipes

\footnotetext{
2 Etablissement public à caractère industriel et commecial
} 
managériales, ce bouleversement à la tête de l'entreprise, avec la CGT comme syndicat majoritaire, a créé des conditions de réflexion dans le champ syndical sur la signification de l'action syndicale : est-ce l'accompagnement d'une modernisation de l'entreprise ? Est-ce un syndicat purement d'opposition mais le cas échéant, comment s'opposer à quelqu'un à la tête de l'entreprise qui a des affinités avec les idées défendues ? Cette situation inédite a ajouté au désordre ambiant et conduit à la fin des années 80 à une situation de blocage, avec une incapacité à s'adapter aux changements de plus en plus fréquents : on constate à cette époque que la demande de transports en commun en Ile-de-France est fluctuante, change fréquemment et ne répond pas forcément à des logiques de moyen/long terme, ce qui appelle une nécessité évidente d'adaptation. Or qui dit capacité d'adaptation, dit capacité de changer les conditions de travail des salariés. Cette équation s'avère forcément compliquée dès lors que les décisions sont prises en haut, et que l'on évolue dans une culture d'opposition.

\section{Une modernisation salutaire}

La grande chance est d'avoir atteint cette situation de blocage à la fin des années 80 . Il y a eu alors une conjonction rare, et qui peut-être sauva l'entreprise, de cet état de très fort malaise interne, ressenti à tous les niveaux de l'entreprise, avec d'une part l'intervention d'un homme à caractéristique particulière en la personne de Christian Blanc, et une volonté politique, dans le même temps, d'engager une modernisation du service public en France. Enfin, il y a eu afflux convergent vers la RATP pour en faire une sorte de phare et de symbole de cette modernisation. Cette conjonction a également permis alors de desserrer les contraintes financières de manière à ne pas bloquer l'entreprise en sus. Christian Blanc est donc arrivé en 1990 pour diriger l'entreprise, après avoir réussi au préalable une mission très délicate en Nouvelle-Calédonie en conciliant l'inconciliable sur un terrain politique extrêmement miné. C'est ce qu'il a fait dans l'entreprise et a réussi, en tout cas jusqu'à ce jour. Sa première action a été de complètement changer la structure, en un an, sur la base de constats existants qu'il a renforcés. Il a ainsi réduit à 3 les niveaux hiérarchiques d'une entreprise qui en possédait entre 7 et 9 , selon les estimations. Mais la chance de l'entreprise est que la contrainte qui l'a animée, est venue alors de l'intérieur plus que de l'extérieur: le souci du client, la perspective de l'intégration européenne, les difficultés à venir liées à la concurrence, l'incapacité d'alors à s'y préparer. Tous ces éléments ont bien servi de support à la communication, mais la réalité simultanée était le malaise fondamental vécu à tous les niveaux et qui se voyait par tous les pores de l'entreprise. Cette situation, qui était proche du désespoir dans certains cas, peut être considérée comme la grande chance de l'entreprise, car elle a permis de capitaliser l'ensemble des énergies. A quelques exceptions près, l'entreprise s'est alors mise en marche.

Les conséquences ont été une décentralisation, une responsabilisation managériale et donc une activité en termes de ressources humaines qui entraîne l'introduction de contrats d'objectifs, la responsabilisation des parcours professionnels, qui était alors quasi impossible avant, l'introduction d'une part variable de rémunération, et donc une incitation à la fois salariale et liée à la richesse de l'activité. 


\section{Un dialogue social apaisé et rénové}

Plusieurs étapes se sont succédées pour arriver à la période actuelle : la réforme des structures, les impulsions de la dynamique managériale et, dans le même temps, un accompagnement des organisations syndicales pour faciliter leur évolution. Effectivement, de la même manière, la structure bureaucratique existait bel et bien des deux côtés de la table. Le "centralisme démocratique», pour reprendre une expression souvent employée à une certaine époque, fonctionnait très bien. Il y avait une sorte de phasage entre la façon de fonctionner d'un côté et de l'autre, qui correspondait à certaines évolutions culturelles. Evidemment, lorsque l'on change la structure et que l'on adopte des comportements différents d'un côté de la table, le jeu d'acteurs doit aussi changer sous peine de ne plus fonctionner. Il y a donc eu un accompagnement basé essentiellement sur la formation et l'information, parfois aussi coercitif avec l'établissement de jurisprudences, notamment sur l'utilisation des préavis de grève, leur rédaction et leur utilisation, afin de recaler les pratiques dans la réalité des lois existantes, au regard des dérives qu'il y avait pu avoir. Cet accompagnement a été également un travail informel de sensibilisation avec les organisations syndicales qui souhaitaient évoluer dans leurs pratiques. On a rencontré un écho favorable dans la plupart d'entre elles, ce qui a permis au bout de cinq ans (1990-1995), quelques mois après la grève de 1995 qui avait paralysé les transports en commun d'Ile-de-France, de formaliser en mai 1996 les règles du dialogue social au travers d'un protocole qui a pour point central la préoccupation du client.

C'est autour de cette notion que l'on a en effet réussi à établir un constat commun et des pistes d'évolution. Ce protocole contenait d'ailleurs à l'époque une partie nommée « code de déontologie ». On s'était alors accordé sur la philosophie de départ des relations sociales et pas seulement sur les modalités de leur exercice. C'est à ce moment qu'a été inventé le dispositif d'alarme sociale, dispositif de prévention des conflits qui peut permettre d'éviter l'utilisation d'un préavis de grève. Il faut rappeler qu'en France, le préavis de grève a été pendant de longues années le seul moyen pour les organisations syndicales d'exprimer leur mécontentement voire d'obtenir un échange avec certaines directions d'entreprise (800 à 900 préavis de grève par an à la RATP à la fin des années 80). La mise en place de règles structurées de concertation et de négociation a abouti à la situation actuelle de relative stabilité, qui a permis de diviser par cinq la conflictualité à la RATP. La négociation collective qui, en 1996/1997, produisait sept accords par an, a permis au cours de chacune des deux dernières années, d'en conclure 42 , issus pour beaucoup de la négociation collective locale. Cette dynamique permet aujourd'hui, compte tenu des règles qui se sont installées et que l'on a pratiquées de part et d'autre et du niveau relationnel qui s'est établi, d'échanger des informations, y compris stratégiques, au Conseil d'Administration, susceptibles d'anticiper les évolutions de long terme. Ce sont par exemple l'automatisation des lignes de métro ${ }^{3}$ ou encore la disparition progressivement testée des tickets de métro au profit d'une carte à puce servant de passe, qui aboutit socialement à la disparition quasi générale de l'ensemble des guichets. Mais cette situation a été anticipée suffisamment à l'avance, en partenariat avec

\footnotetext{
3 Antérieurement, ce sujet aurait provoqué l'arrêt immédiat de l'ensemble des lignes de métro, alors qu'aujourd'hui une ligne est automatique et une autre sera automatisée sans que cela ait donné lieu à des conflits sociaux
} 
les organisations syndicales, pour que puissent être abordées et mises en place, y compris par des expérimentations, ces évolutions technologiques, managériales et organisationnelles. Cet ensemble d'évolutions structurelles nous permet aujourd'hui d'estimer que la RATP est préparée à la mise en concurrence qui s'approche et à laquelle l'entreprise s'entraîne déjà par les réponses à des mises en appel d'offres. Certains ont été remportés, certains ont conduit à l'échec mais la différence s'est en général faite sur d'autres critères que des critères purement économiques ou organisationnels. L'entreprise se sent en cela prête à aborder le 'vrai marché', sans que cela génère pour autant des craintes élevées chez les salariés. Aujourd'hui, les craintes qui se font jour, telles qu'elles sont appréhendées par des enquêtes, s'expriment moins sur le statut du salarié que sur le statut de l'entreprise.

VOILÀ EN QUELQUES MOTS comment, par l'effet d'une conjonction exceptionnelle et d'un grippage généralisé, on a peut-être créé des conditions permettant non pas de se rapprocher d'une normalisation des relations sociales, même si selon les termes qu'elle emprunte, la RATP souhaite être une entreprise à part entière, mais en ayant su créer, compte tenu d'une histoire particulière, une spécificité des relations sociales dont on n'exclut pas complètement de faire un atout concurrentiel pour la suite. 



\title{
La représentation du personnel, acteur de la modernisation? Expériences de pilotage partagé de la réforme
}

\author{
Hermann HIBELER
}

Dix années de représentation des intérêts du personnel dans une municipalité en mutation laissent des traces et transforment durablement le rôle et les méthodes du Personalrat (l'instance de représentation du personnel). Les dix années de travail de l'auteur comme président du Personalrat de la ville de Detmold et son expérience de la réforme de l'administration constituent la base des réponses à la question : " la représentation du personnel peut-elle être un acteur de la modernisation?»

Les expériences ont été réalisées à Detmold, ville moyenne de la République fédérale (75 000 habitants), dans l'Est de la Rhénanie du Nord-Wesphalie (NRW), qui emploie environ 1000 personnes. Le nouveau modèle d'organisation de la ville se base fortement sur le «nouveau système de pilotage » développé par la KGSt (Kommunale Gemeinschaftsstelle, Cologne). A Detmold comme ailleurs, on pensait que seule une gestion plus efficiente et plus efficace de l'administration permettrait de dégager à l'avenir de nouvelles marges d'action. Cette idée s'appuyait sur l'hypothèse selon laquelle l'administration centrale était trop encadrée, et les participations pas assez. Detmold s'est aussi très largement inspirée de modèles d'organisation des entreprises privées (la ville comme grande entreprise). Cette représentation - qui, comme nous le verrons, n'était pas tout à fait sans poser problème - était certainement aussi influencée par l'activité professionnelle des acteurs du processus de transformation. A Detmold, les modalités d'application ont été caractérisées, outre par les acteurs, par certaines conditions qui ont marqué et accéléré les résultats. Les personnes, les conditions et la stratégie ont également fortement influencé la représentation des intérêts du personnel, le Personalrat. Ses expériences du processus de réforme doivent être resituées dans ce contexte.

\section{Le contexte général}

La ville de Detmold est un centre administratif et de services, d'orientation plutôt conservatrice. L'action de l'administration était perçue avec une certaine passivité, ses prestations n'étant donc pas soumises à la critique, et le budget municipal était équilibré grâce à une gestion solide. Les difficultés financières ne constituèrent donc pas le principal moteur des processus de modernisation. A l'inverse, le Stadtdirektor (chef des services administratifs municipaux) et quelques rares cadres, mus par le souci d'assurer durablement la capacité d'action de la ville, ont joué un rôle décisif. Le passage de Detmold, au milieu des années 1990, de sa quiétude contemplative à une entrée en force 
dans l'ère des réformes, s'explique par trois facteurs, qui constituèrent conjointement la « masse critique » indispensable au démarrage.

\section{La « masse critique de départ » comme facteur d'impulsion}

Le premier facteur décisif fut la présence d'acteurs ayant la vision d'une administration moderne, efficace. Comme il s'agissait des principaux décideurs, disposant de pouvoir et d'influence, ils étaient en situation d'imposer leur vision. Ils avaient aussi la liberté d'admettre une « culture de l'erreur ». Ce n'est pas la sécurité comme résultat d'une planification détaillée qui était visée, mais priorité était donnée à l'essai et à l'expérimentation. Cette culture, nouvelle pour une administration publique, libéra une énorme motivation latente.

Cette évolution fut rendue possible par un changement d'équipe, avec l'arrivée d'une autre génération disposant de nouvelles qualifications. Le Stadtdirektor et sa première adjointe, nouvellement élus à peu d'intervalle, venaient de l'extérieur, étaient jeunes, diplômés en économie, et s'ils avaient une expérience relativement modeste de l'administration, leur expérience politique était d'autant plus solide. Leur élection constitua une césure, car leurs fonctions étaient traditionnellement une chasse gardée des juristes. ${ }^{1}$

La situation géographique constitua un deuxième facteur. La région avait été le théâtre de débats sur la réforme de l'administration, et des projets pilotes y avaient été soutenus. Les modèles et inspirateurs furent notamment la ville de Tillburg aux PaysBas, la KGSt avec son directeur M. Banner et la fondation Bertelsmann, qui soutenait fortement plusieurs projets pilotes dans la région, et qui, plus tard, organisa conjointement avec la fondation Hans-Böckler et la KGSt le réseau «Communes de l'avenir », auquel la ville de Detmold participa avec plusieurs projets. Les projets «L'avenir grâce aux services publics », du syndicat des services publics et des transports (ÖTV), donnèrent des pistes de réflexion et des impulsions utiles au Personalrat.

Les prévisions économiques constituèrent le troisième facteur décisif: si les difficultés budgétaires n'étaient pas encore d'actualité, une détérioration générale des finances locales s'annonçait à moyen terme pour les municipalités. Car, à long terme, la participation des communes au financement de la reconstruction des nouveaux Länder, l'évolution durablement négative du marché de l'emploi et de l'activité économique et les dépenses en forte hausse au titre du système de protection sociale allaient, selon les estimations, peser considérablement sur les budgets municipaux. Pour pouvoir conserver à l'avenir une capacité d'action politique, il fallait une organisation plus efficace et plus efficiente. Les éléments moteurs de la réorganisation devaient être la transparence, la concurrence et la participation. Il fallait également contrôler davantage les objectifs et les résultats, pour mieux « prendre en main » les problèmes.

A Detmold, les difficultés financières, qui apparaissent souvent comme le principal moteur de la modernisation des administrations, jouèrent donc dans un premier temps un rôle indirect et secondaire. Les administrés et les personnels ne furent pas plus décisifs, aucune critique significative n'émanant de leur part. De ce point de vue non plus, Detmold n'avait donc pas matière à faire la une de la presse. Mais cette situation changea fondamentalement à partir de 1995 : après deux années de préparation interne, Detmold gagna, avec son projet de réforme, l'intérêt du public.

\footnotetext{
${ }^{1}$ Deux autres changements de direction ultérieurs corroborent la thèse selon laquelle les personnes exercent une influence importante, voire décisive, sur l'orientation et la rapidité des processus de réorganisation.
} 
Comme d'autres villes, Detmold choisit d'abord des domaines pilotes, pour expérimenter dans la pratique les idées développées. C'est la démarche adoptée qui donna son dynamisme au processus de changement et l'accéléra : mis à part quatre objectifs principaux, aucune contrainte ne fut imposée lors du processus de réalisation, ce qui laissait une grande marge de décision.

La démarche

La démarche fut déclarée expérimentale, ce qui ouvrait tout un éventail de solutions possibles, laborieusement regroupées au sein d'un comité de pilotage (instance de décision). Bien qu'une enquête effectuée en début de parcours attestât d'une grande satisfaction chez les collaborateurs, une insatisfaction latente existait manifestement parmi les employés, et celle-ci, associée à des attentes élevées, débouchait sur un fort désir d'implication. Les attentes étaient surtout nourries par la perspective d'une participation accrue et d'une plus grande liberté de décision, ainsi que par celle d'une amélioration des conditions de travail. La volonté de participer aux groupes de travail fut donc importante et la charge supplémentaire afférente acceptée sans protestation. Le Personalrat demanda une compensation pour le travail supplémentaire, mais ne trouva aucun soutien chez les personnels concernés. Sous la pression de la demande, la direction générale des services dut rapidement renoncer à sa stratégie, consistant à expérimenter les idées de changement dans quelques domaines pilotes, pour intégrer l'ensemble de l'administration. Le Personalrat se rallia lui aussi à l'unanimité à ce projet.

Le $1{ }^{\text {er }}$ janvier 1995, une organisation de l'administration restructurée se mit donc en place. Elle attira l'attention au niveau régional et national, car une administration municipale sans découpage classique en plusieurs services n'avait jamais existé ; c'était quasi-inimaginable.

Tableau 1 : organisation administrative de la ville de Detmold

Jusqu'en 1995

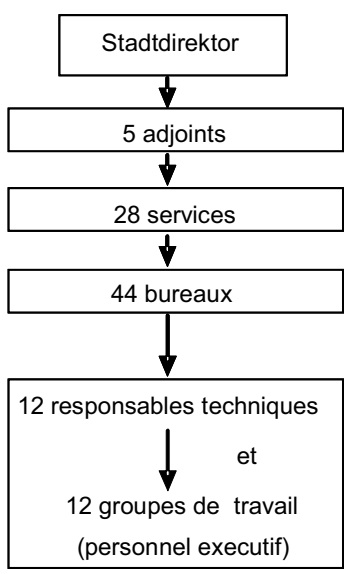

A partir de 1995

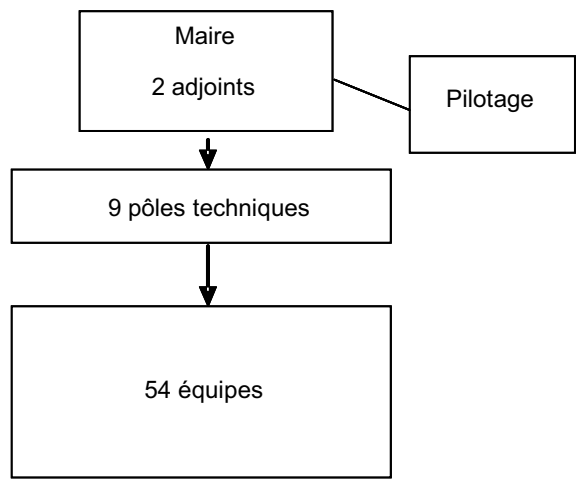

Le nombre de commissions passe à 10 , soit une réduction de moitié 


\section{Description des processus et objectifs}

Cet ambitieux projet de restructuration, radical pour la ville de Detmold, fut précédé d'une période préparatoire de deux ans, au cours de laquelle il fut débattu, sans contrainte ni limite, d'une organisation administrative visionnaire. Au début, rares furent ceux qui participèrent à ce débat, porté uniquement par quelques cadres et responsables de services. C'est dans ce cercle que furent développées les visions 1993 1998, comportant quatre objectifs principaux que les employés furent invités à discuter dans le cadre de forums. Suivit ensuite un processus participatif en ateliers auquel tous les salariés eurent la possibilité de se joindre, sur la base du volontariat, durant leur temps de travail, une occasion que saisirent la majorité d'entre eux.

Quatre objectifs (principaux)

On commença par discuter des quatre objectifs principaux (visions) pour la période 1993-1998 :

- Amélioration de la qualité des prestations et du service ;

- Augmentation de la productivité ;

- Optimisation des conditions et processus de travail ; humanisation du travail ;

- Amélioration de l'image de l'administration.

A l'instar de l'objectif d' " amélioration de la qualité des prestations », ont été développés lors du processus de discussion les sous-objectifs suivants :

\section{Sous-objectifs}

- Meilleur accueil des administrés ;

- Meilleure prise en compte des souhaits des administrés ;

- Souci de qualité dans l'action administrative ;

- Espaces de conseil et de travail respectueux des administrés ;

- Décisions pertinentes et prises dans des délais rapides

Pour chacun de ces sous-objectifs, des propositions de mesures ont été élaborées en concertation avec des salariés concernés. Sur l'initiative du Personalrat, les propositions ont pour une part été formulées dans une grande autonomie par des cercles de qualité (CQ). Cette méthode, d'abord rejetée par les instances dirigeantes, fut finalement acceptée en raison de la position du Personalrat. Cette réserve initiale s'explique par le fait que les personnels d'encadrement ne devaient en principe pas être associés ou bien, le cas échéant, en tant qu'experts et non dans leur rôle de cadres. Les premiers CQ présentant très rapidement des résultats remarquables, très bien acceptés, cette méthode se généralisa très vite.

\section{Au cœur du projet : le guichet unique comme centre multiservices moderne}

Le premier grand projet de restructuration, qui prit une importance décisive pour le déroulement de la réorganisation, fut la conception d'un " Guichet unique ». Cette mesure visait à regrouper sur un site central les services les plus sollicités et de les coordonner de manière à pouvoir traiter chaque demande dans sa globalité. En adéquation avec les situations concrètes, les services relatifs aux déclarations de domicile, cartes d'identité 
et passeports, exonérations de la redevance audiovisuelle, taxe sur les chiens, déclarations de création ou transfert d'activité professionnelle ainsi qu'à l'aide au logement et aux assurances sont organisés de telle manière que l'administré peut depuis lors effectuer ses démarches en un seul endroit. Les files d'attentes habituelles ont ainsi disparu. Comme à la réception d'un hôtel, l'usager peut, au besoin, se renseigner à un accueil central, ou se diriger directement vers l'un des guichets libres. Afin de pouvoir répondre complètement aux besoins des administrés, il a fallu remplacer la division du travail, autrefois importante, par un traitement "global », soutenu par une banque de données réorganisée. Un échelon hiérarchique disparut du même coup, pour être remplacé par une organisation participative en équipes.

L'innovation ne se limita pas à la reconfiguration des lieux d'accueil du public, à des processus spatiaux, organisationnels et à des contenus, mais intégra aussi une extension des horaires d'ouverture, décidée en accord avec les salariés et à la demande des administrés, d'environ 20 heures à 41 heures hebdomadaires. Ceci entraîna une réorganisation de l'emploi du temps des personnels, largement effectuée par le groupe lui-même, et adaptée aux nouveaux horaires ou aux heures les plus chargées. Ce haut niveau d'autonomie fonctionne encore aujourd'hui, dix ans plus tard. Les horaires d'ouverture sont de temps en temps adaptés de manière flexible aux variations de fréquentation. Dans les enquêtes auprès des administrés, ce service est particulièrement bien noté. Le Personalrat a apporté son soutien exprès à ce projet, d'une part en raison de la très large suppression de la division des tâches et de la modernisation des conditions de travail, mais surtout de la possibilité donnée aux employés de s'autoorganiser.

Cette mesure, qui a permis à l'administration municipale de donner l'image d'un service moderne, a non seulement augmenté la satisfaction des administrés, mais aussi la valorisation du personnel, ce qui a eu des répercussions sur les autres domaines de l'organisation administrative.

Une approche globale qui met fin à la division du travail et au manque de visibilité

Ce projet a clairement montré qu'il existait une alternative attractive à l'organisation jusque-là cloisonnée et hiérarchique de l'administration, répondant à la fois aux nouvelles exigences d'efficacité et au souhait d'une participation accrue. C'est pourquoi, au sein du comité de pilotage, composé de représentants de la hiérarchie administrative des directions des pôles techniques, du Personalrat, et au sein des groupes de projets, des voix se sont élevées pour demander que l'organisation soit structurée en fonction des procédures, de l'extérieur vers l'intérieur, donc à partir des besoins et intérêts des administrés. Pour y parvenir, on débattit du remplacement de la division du travail par ce qui fut appelé le traitement global. Car, sans ce changement, il n'y avait pas de place pour le concept de "guichet unique » répondant à l'ensemble des besoins des administrés. L'objectif fut formulé ainsi : c'est aux dossiers et non aux personnes de se déplacer. Cette base permettait de concilier l'objectif organisationnel de satisfaction des administrés avec la suppression de la division du travail réclamée par le Personalrat. Pour atteindre ce double objectif, il fallait un processus ambitieux et porté collectivement, afin d'analyser avec les personnes concernées les processus de travail, d'établir des synergies judicieuses, de développer un soutien technique et de mettre en place les formations adéquates. 


\section{Engagement et persévérance}

Les cadres et le Personalrat durent faire preuve de longue haleine, ce qui apparut en particulier dans l'exemple de la réorganisation du bureau de l'état civil. Au fil d'un processus de plusieurs mois, qui suscita d'abord le rejet violent par des personnes concernées, fatalistes, on parvint à trouver un compromis satisfaisant pour les administrés et pour les employés, qui générait en outre des économies. La persévérance et la participation conséquente des personnels concernés, ainsi que la patience de la direction des pôles techniques et du Personalrat réussirent à transformer les expériences négatives en une attitude durablement positive. Non seulement l'opposition éventuelle du Personalrat n'aurait rien changé aux inégalités des charges de travail et aux structures organisationnelles développées sur la durée, mais elle aurait pérennisé cette situation insatisfaisante, si bien que l'ambiance se serait encore dégradée. Maintenant, les processus de travail sont organisés de manière plus globale, il n'existe plus de doublons; volumes et temps de travail sont adaptés les uns aux autres, et l'ambiance s'est considérablement améliorée.

\section{Des missions aux produits}

Comme il fallait de toute façon redéfinir les missions et prestations de toute l'administration et les qualifier de produits, ceux-ci furent ensuite rassemblés en groupes de produits ayant des liens concrets entre eux. Au plan organisationnel, ces ensembles furent regroupés en pôles techniques et dotés d'un budget annuel ainsi que d'objectifs à atteindre. Ces unités devaient donc réaliser leurs résultats sous leur propre responsabilité et en rendre compte à la hiérarchie (jusqu'alors à la direction des services) et au conseil municipal dans des rapports trimestriels. Les excédents devaient pour une part être affectés au pôle technique, récompensant ainsi ses performances. Les premières années, cette réussite du management fut elle aussi portée pour une part $(30 \%)$ au crédit du budget technique correspondant. Avec la détérioration croissante de la situation financière de la ville, les responsables politiques intervinrent et reprirent la main sur l'utilisation de ces moyens.

\section{Réduction de la hiérarchie et participation}

L'étape suivante consista logiquement à créer, à partir des 28 services existants, 8 pôles techniques plus un service de vérification des comptes (instance obligatoire) et, parallèlement, à réduire le nombre de commissions spécialisées du conseil municipal. Cette étape ne fut possible que parce que plusieurs cadres âgés quittèrent leurs fonctions. Le Conseil apporta son soutien unanime. Le projet de modernisation s'avança ici, pour la première fois, sur un terrain dangereux. La réduction du nombre de postes de chef de service restreignait les perspectives de progression, ce qui fit naître des craintes chez les cadres désormais en surnombre. Le processus de réforme créa ainsi un « potentiel d'opposition » latent. Les pôles techniques devaient être divisés en équipes partiellement autonomes, mises en place en accord avec le Personalrat. Cette étape remettait en question 44 directions de bureaux. L'une des exigences du Personalrat - faire élire la direction de l'équipe par le groupe lui-même - renforça d'abord cette inquiétude. La direction des services n'aurait jamais accepté cette demande, sans doute par crainte que les chefs de bureaux soient évincés. Mais, à une exception près, les équipes, suivant 
l'appel du Personalrat, élirent - avec l'indulgence des directions des pôles techniques, parfois par vote secret - les chefs déjà en fonction. Si les craintes de la direction des services s'avéraient ainsi infondées, le rapport entre chefs d'équipes et salariés se trouva toutefois considérablement transformé. Les décisions étaient prises et assumées de manière plus collective.

\section{Un processus soutenu aussi par le conseil municipal}

Pour le conseil municipal, cette simplification impliquait une réduction de moitié du nombre de commissions spécialisées, ce qui diminuait du même coup le nombre de fonctions de direction prestigieuses. La perte d'influence sur des questions de détail pesait encore plus lourd, puisque, d'après le nouveau modèle de pilotage (NSM), la politique devait se concentrer sur le contenu, et les pôles techniques de l'administration, sur les modalités. La direction de l'administration, composée du Stadtdirektor et de trois (puis deux) adjoints, devait, selon ce modèle, se considérer comme une instance collégiale et se défaire des compétences spécialisées détenues jusque-là, afin d'assurer collectivement le pilotage de la nouvelle organisation et de définir sa stratégie. Les politiques s'écartèrent ensuite encore souvent des modalités convenues et tentèrent de se décharger de leurs responsabilités. Il leur était tout aussi difficile de cesser d'intervenir directement que de porter conjointement la responsabilité des missions et des dépenses. Même les budgets et catalogues de missions définis pour une période d'un an furent considérés comme une entrave à l'action politique.

\section{La participation du Personalrat}

L'ensemble du processus de transformation fut accompagné, depuis sa toute première phase de développement, donc depuis le débat sur le projet, par le Personalrat. Au-delà des obligations légales (Loi du Land NRW sur la représentation du personnel) cette instance fut associée dès la toute première phase de conception des mesures, ses représentants siégeant et votant dans les différents comités, y compris le comité de pilotage. Cette forme de participation précoce et directe fut toutefois source de difficultés pour le Personalrat. En effet, le processus de transformation était conçu de manière ouverte, c'est-à-dire sans préjuger des résultats, exception faite de l'objectif général d' "augmentation de la performance de l'administration ». Les expériences étaient explicitement encouragées, et, quelle qu'en fût la forme, elles furent finalement perçues comme des moyens de trouver la voie la plus appropriée.

\section{Changement dans la représentation du personnel}

Ni les employés ni le Personalrat n'avaient l'expérience de tels processus ouverts de développement des organisations. Chez une partie du personnel, la peur d'un avenir qui ne leur semblait plus prévisible se fit sentir. Un groupe assez important de salariés développa quant à lui des attentes qui soit étaient irréalistes, soit allaient contre les intérêts d'autres groupes. Il s'avéra difficile pour le Personalrat de réconcilier ces intérêts divergents, d'autant qu'il ne pouvait s'engager à donner des informations fermes sur l'avenir et sur les conséquences des processus de transformation. Il se trouva donc pris en étau entre euphorie, inquiétude, scepticisme et éloignement, et dans une dissension des intérêts qui le menaçait d'implosion. Il risquait de perdre toute vision 
d'ensemble et des répercussions du projet. Ni la réalisation des objectifs ni l'impact sur la sécurité de l'emploi, des revenus, les perspectives d'évolution et les charges de travail ne pouvaient être déterminés.

\section{Légitimation de la représentation du personnel}

Il apparut ici que la loi sur la représentation du personnel n'était qu'insuffisamment adaptée à des processus aussi participatifs. La législation en vigueur part du principe d'une participation «en aval». Après avoir été élaborée par la direction des services, une mesure est présentée au Personalrat: celui-ci doit disposer de l'ensemble des informations nécessaires à la prise de décision; il se concerte ensuite au sein de ses instances dans de brefs délais et en suivant les "intérêts du service et des salariés ». Il décide alors, à la majorité de ses membres, d'accepter ou de refuser. S'il estime qu'il lui manque des informations, il prend la décision de « ne pas accepter délibérément », afin de discuter de cette question avec le service et de trancher ensuite. En cas de nouveau refus, une instance de conciliation peut être convoquée : composée de trois représentants de chaque partie (personnels et employeur), elle prend, sous la direction d'un président neutre, une décision qui peut alors, en dernier recours, être acceptée ou rejetée par le conseil municipal.

Cette procédure suit des modalités de décision très hiérarchiques et n'associe généralement pas le Personalrat à l'élaboration des mesures. Selon la loi sur la représentation du personnel, la mission de développement de l'organisation peut être définie dans le cadre d'une procédure de co-décision avec le Personalrat. Mais il n'est en fait plus possible de définir précisément les répercussions et toute une série de réalités relevant de la co-décision. En outre, une fois la décision prise par consensus au sein de l'équipe de développement de l'organisation, il ne reste pas de marge de décision réelle pour le Personalrat, qui doit prendre position dans le sens de l'intérêt général (par exemple emploi de personnes souffrant d'un handicap léger ou lourd, protection du travail et de la santé, lutte contre la discrimination, etc.).

\section{Processus de transformation dans la représentation du personnel}

Cette méthode signifiait que le Personalrat devait participer à un processus auquel il n'était pas préparé et pour lequel il ne disposait pas des outils adéquats. Il travailla conformément à ses expériences et à sa mission légale, demandant au directeur des services d'expliciter les objectifs concrets de la réorganisation et de se prononcer sur ses conséquences. Il reçut en réponse les objectifs cités plus haut et on lui indiqua que les répercussions sur le personnel seraient gérées « dans le respect des exigences sociales ». Mais, les objectifs étant plutôt flous et le processus volontairement conçu comme ouvert et participatif, le Personalrat ne pouvait pas entreprendre grand-chose. Les représentants du Personalrat déjà impliqués dans le processus et les personnes y participant activement s'éloignèrent de cette stratégie classique de l'instance de représentation, ne voulant en aucun cas revenir à une action encadrée par des procédures ou limitée à des mesures au cas par cas. Le Personalrat entra ainsi dans un conflit qui menaçait de le diviser. La réélection du Personalrat tomba à ce moment-là, ce qui entraîna des changements de personnes au sein même de la structure. L'élection mit un peu de distance par rapport au conflit et donna lieu à un bilan critique de la stratégie du Personalrat et, par conséquent, à une reformulation des contenus et des stratégies. 


\section{Changement de stratégie du Personalrat}

Les nouveaux élus prirent conscience que continuer comme avant entraînerait à moyen terme une perte de crédibilité du Personalrat et que, dans des processus modernes de développement de l'organisation, une participation représentative strictement conforme aux règles, intervenant en aval, conformément à la législation sur la représentation du personnel, ne suffirait pas à elle seule à assurer une représentation efficace du personnel. Si la participation directe continuait de se développer, elle nuirait à la participation collective classique, car ce qui était décidé sur place, à l'amiable, entre les acteurs concernés, pourrait difficilement être remis en cause au niveau « supérieur » par le Personalrat, même sur la base du dispositif réglementaire de co-décision. De plus, l'incertitude grandissante quant aux conséquences possibles, ainsi que le conflit qui se dessinait peu à peu entre les «modernisateurs » et les «traditionalistes » allaient faire apparaître des intérêts très différents, difficilement conciliables par le Personalrat dans le cadre du processus participatif.

\section{Positionnement}

Concrètement, le Personalrat devait se positionner et choisir :

- d'empêcher ;

- de fermer les yeux ;

- de se résigner ;

- de (co-)décider.

Les deuxième et troisième alternatives n'entraient pas sérieusement en considération. Dans ce processus de positionnement, le Personalrat arriva majoritairement à la conviction que, compte tenu du large soutien du personnel, seule une stratégie " proactive » pouvait fonctionner, et opta pour une logique du «contre-pouvoir par la co-décision ». Un autre élément fut décisif : la prise de conscience que les objectifs de transformation pourraient se combiner à ceux des employés ou du Personalrat, s'ils étaient intégrés de manière active au processus et représentés par des méthodes adéquates.

\section{(Nouveau) rôle du Personalrat}

Ce processus d'évolution du Personalrat avait pour base six (hypo)thèses (ou encore « constats »), indiquant une nécessité d'agir évidente, voire urgente dans certains cas :

- Le besoin de changement est considérable, en particulier dans l'organisation des procédures et la gestion des ressources humaines ; cela signifie que la hiérarchie, très cloisonnée jusquelà, a entraîné l'hétéronomie et doit laisser place à davantage de participation et d'auto-organisation (démocratie sur le lieu de travail);

- Pour une grande partie des prestations administratives, la concurrence est de plus en plus importante et rude, soutenue qui plus est par des motifs idéologiques qu'un manque de transparence et donc de possibilités de comparaison empêche de réfuter de manière crédible ( «les prestataires privés sont plus efficaces que le service public bureaucratique »);

- On constate des tensions latentes entre usagers et administration à cause d'une organisation ; - Les différences entre performances de l'équipe dirigeante attendues et effectives sont considérables. C'est de là que provient une large part des conflits qui occupent le Personalrat ; 
- Le travail du Personalrat présente un déficit d'efficacité - et pas seulement du fait de la loi sur la représentation du personnel - qui l'expose à un risque de perte de crédibilité ;

- Les intérêts des trois camps de salariés - les perdants, les gagnants et les hésitants - sont nettement divergents, mais tous doivent être représentés de la même manière. Cela signifie en définitive que le rôle du Personalrat doit à l'avenir à la fois consister, en fonction des situations, à garantir, soutenir, conseiller et, si nécessaire, empêcher.

Le Personalrat a fait suivre ces appréciations d'une redéfinition de sa stratégie, afin de faire des propositions pertinentes, qui lui permettent d'être perçu par un maximum de salariés comme une instance de représentation efficace de leurs intérêts. Faute de connaître les possibles répercussions de la réforme, le Personalrat n'était toutefois pas en mesure d'apporter des solutions pertinentes pour répondre aux craintes. La direction des services laissant à dessein le processus de transformation ouvert et ne pouvant ou ne voulant pas en prévoir l'impact, l'instance de représentation du personnel dut se charger elle-même de cette tâche, afin de trouver une base pour proposer des alternatives ou motiver ses refus.

\section{Stratégie du Personalrat}

Lors d'une réunion du Personalrat, le rôle de co-décideur à venir a été définie et des priorités arrêtées sur la base de questions hypothétiques et dans un processus en trois étapes :

- Analyse de l'impact de la modernisation sur le personnel (sécurité de l'emploi, conditions de travail, revenus, statut, qualifications). Cette étape analytique tentait également de définir les gagnants et les perdants potentiels.

- Analyse de l'impact de la modernisation sur la représentation du personnel par la démarche participative (participation directe des personnels concernés) et de celui de la délégation des responsabilités, de l'aplanissement des hiérarchies.

- Définition du rôle et des objectifs du Personalrat pour permettre une participation active et efficace.

Une autre séance a évalué les tensions perceptibles chez le personnel, afin de classer les résultats d'analyse en fonction et de définir les futurs champs d'action.

Tableau 2 : Tensions dans le processus de transformation

\begin{tabular}{|c|c|c|c|}
\hline Situation de départ & Attentes antérieures & Objectifs de transformation & Effets (attendus) \\
\hline $\begin{array}{l}\text { emploi à vie, sécurité } \\
\text { de l'emploi }\end{array}$ & $\begin{array}{l}\text { sécurité, prévisibilité, } \\
\text { progression, plan de } \\
\text { carrière }\end{array}$ & rentabilité & $\begin{array}{l}\text { risques de perdre face à la } \\
\text { concurrence, déréglementation }\end{array}$ \\
\hline $\begin{array}{l}\text { hiérarchie, devoir } \\
\text { d'obéissance }\end{array}$ & $\begin{array}{l}\text { encadrement, } \\
\text { adaptation }\end{array}$ & $\begin{array}{l}\text { décentralisation des respon- } \\
\text { sabilités, priorité à l'accomplis- } \\
\text { sement de la mission confiée }\end{array}$ & $\begin{array}{l}\text { responsabilité individuelle, } \\
\text { risques liés à la prise de } \\
\text { décision, qualifications }\end{array}$ \\
\hline $\begin{array}{l}\text { règlementation très } \\
\text { précise, priorité au res- } \\
\text { pect des normes, même } \\
\text { pour les prestations }\end{array}$ & $\begin{array}{l}\text { encadrement, peu de } \\
\text { responsabilité } \\
\text { individuelle }\end{array}$ & priorité à l'administré & $\begin{array}{l}\text { risque liés à la prise de déci- } \\
\text { sion, responsabilité indivi- } \\
\text { duelle, flexibilité, mobilité }\end{array}$ \\
\hline $\begin{array}{l}\text { contrôle et réglemen- } \\
\text { tation très denses }\end{array}$ & encadrement, sécurité & $\begin{array}{l}\text { qualité des missions, degré } \\
\text { d'efficacité }\end{array}$ & $\begin{array}{l}\text { apprentissage permanent, } \\
\text { remise en question }\end{array}$ \\
\hline
\end{tabular}


L'étape suivante a consisté pour le Personalrat à définir six champs d'action :

- Sécurité de l'emploi ;

- Qualification ;

- Employabilité ;

- Autonomie et participation des employés « à leur niveau »;

- Contrôle des changements ;

- Compétitivité.

Avec ces six champs d'action, le Personalrat, garant de stabilité, a voulu, face au manque de visibilité du nouveau système d'organisation pronostiqué, donner un ancrage à la sécurité légale de l'emploi, et fournir aux personnels concernés les formations et l'optimisation des conditions de travail nécessaires, afin de leur donner confiance dans les possibilités de planifier l'avenir et dans leurs propres capacités à faire face aux changements.

L'une des principales préoccupations du Personalrat a été de démocratiser l'organisation en supprimant la hiérarchie et l'hétéronomie. Le contrôle des changements devait permettre de documenter le processus de transformation afin d'empêcher les dérives.

Le Personalrat associait au champ d'action "compétitivité » l'idée qu'une concurrence menée avec des règles (du jeu) transparentes et justes rendrait les emplois plus sûrs, d'autant plus que la fonction publique ne pourrait, à court ou long terme, se soustraire à la comparaison avec le secteur privé. Mais ce thème fait peur. Trop d'expériences négatives d'un côté et d'attentes idéologiques de l'autre ont freiné une confrontation ouverte avec la question de la compétitivité.

\section{Mesures et initiatives du Personalrat}

Le Personalrat a croisé avec les six champs d'action les initiatives suivantes, garanties juridiquement dans des conventions de service :

1. Sécurité de l'emploi : le Personalrat s'est entendu avec la direction des services sur une garantie d'emploi, liée à une certaine obligation pour les employés de se former si nécessaire pour de nouvelles missions. Les licenciements économiques sont ainsi exclus. Cet accord comporte également une condition mettant les relations internes de prestations (prestations annexes) sous obligation de contracter durant une période d'adaptation de cinq ans (protection contre l'attribution à des prestataires privés).

2. Sécurité de l'emploi : sur l'initiative du Personalrat, un marché interne de l'emploi, la « bourse du travail », a été organisé sur la base d'une convention de service afin de maintenir, grâce à des mesures de formation, l'employabilité des personnels affectés par la réorganisation ou la suppression de leurs missions. Cette mesure devrait permettre de répondre à la hausse de productivité souhaitée par le Personalrat et ouvrir pour les personnels concernés de nouvelles perspectives d'emploi. Elle a été imposée contre les fortes résistances de la direction, et l'on courait initialement le risque que les personnels supposés ou réellement peu productifs soient rejetés vers la «bourse du travail ». La réponse du Personalrat a consisté à ne plus accepter de recrutements sans passer par la bourse du travail, afin de valoriser cette dernière. Cette condition garantissait que tous les employés souhaitant changer de poste ne le feraient que par ce biais.

3. Sécurité de l'emploi et employabilité : un accord a été trouvé pour promouvoir l'état physique des salariés. Il s'agit de supprimer les conditions de travail pénibles, d'inciter les salariés à un comportement adéquat de nature à réduire les jours d'absence et de maintenir 
leurs capacités physiques et psychiques. Une analyse des congés maladie, demandée par le Personalrat, a en effet montré un taux d'absence pour maladie nettement supérieur à la moyenne, avec un impact négatif, d'une part en termes de compétitivité (coûts vraisemblablement plus élevés), et d'autre part en termes de charges de travail supplémentaire considérables. En outre, selon l'évaluation, l'employabilité ultérieure de la personne concernée en pâtirait.

4. Sécurité de l'emploi et qualification : une augmentation du budget formation a été convenue, et de nouveaux outils de gestion des ressources humaines défendus, comme la consultation en matière d'addiction, le coaching, la médiation, l'entretien sur le développement de l'organisation et sur le développement des RH ; ces méthodes, nouvelles pour le service, devraient contribuer à faire accepter les changements prévus et à soutenir la réorganisation.

5. Autonomie/démocratisation : après un processus de discussion difficile qui a pris plusieurs années, le Personalrat a imposé dans une convention que soient définis les missions et le rôle de la direction dans une administration décentralisant les responsabilités. Cette explicitation devait permettre d'assurer, au niveau inférieur de l'organisation, le travail en équipes, partiellement autonomes, souhaité par le Personalrat. Cette mesure résolvait un problème ancien, lié à la décision, portée conjointement par la direction des services et le Personalrat, de réduire la hiérarchie à trois niveaux. Car, après l'euphorie des premiers temps, l'aplanissement de la hiérarchie et la délégation des responsabilités avaient tourné à la déception et au désarroi, d'autant que les personnels étaient insuffisamment préparés à leurs nouveaux rôles. Les craintes suscitées par la suppression d'échelons hiérarchiques et par un nouveau mode d'organisation, davantage tourné vers le marché ou le résultat, étaient à l'évidence trop grandes. Cette situation impliquait en effet des marges de manoeuvre, consistant à prendre des décisions sous sa propre responsabilité, mais avec les risques correspondants, ce qui déstabilisa de plus en plus de cadres. La désorientation était particulièrement présente au niveau des équipes, qui purent participer aux choix de leur direction, mais furent livrées à elles-mêmes dans leurs processus de développement. C'est là qu'intervient l'accord sur la formation des cadres, réalisée sur la base d'un travail collectif de description de certains rôles et missions.

6. Autonomie/démocratisation: un système global d'évaluation a été discuté, qui n'est toutefois pas encore terminé. Le Personalrat a exprimé son scepticisme sur ce projet et sur la possibilité d'exprimer des appréciations objectives. Dans la mesure où les évaluations sont désormais inévitables, suite à la nouvelle convention collective de la fonction publique, un projet à deux dimensions est privilégié : il a été convenu que l'évaluation ne serait pas seulement celle des salariés par les cadres, mais également celle des cadres par les salariés. L'évaluation doit s'inscrire dans un processus balisé. L'évaluation des cadres par les salariés était pour le Personalrat la condition pour accepter les évaluations. Le Personalrat leur préfère toutefois les systèmes participatifs de conventions d'objectifs.

7. Autonomie/démocratisation : les entretiens entre collaborateurs et supérieurs hiérarchiques et les entretiens de convention d'objectifs ont été définis dans une convention, afin de permettre un échange permanent avec les prestataires de services, grâce à des entretiens réguliers sur les objectifs, leur réalisation et leur évaluation, ainsi que sur les conditions des prestations. Le schéma d'action (description idéaltype du processus) est destiné à permettre, en plus de la convention, que cadres et salariés s'entendent, dans un processus participatif, sur les objectifs, la mobilisation des ressources et l'organisation, qu'ils évaluent les résultats et, le cas échéant, les réajustent ensemble.

8. Contrôle des changements : il a été convenu de faire au bout de cinq ans le bilan des répercussions de la réorganisation sur les salariés, par le biais d'une enquête auprès des collaborateurs. La représentation du personnel a en outre imposé une évaluation de l'ensemble du processus de transformation par des chercheurs indépendants. Ces mesures sont destinées à permettre de contrôler de manière critique l'impact du processus de transformation 
et d'apprécier à leur juste valeur les avis parfois contradictoires sur l'état de réalisation. Il faut le cas échéant fixer de nouvelles priorités pour compenser les insuffisances. Le résultat de cette évaluation a ainsi largement aidé le Personalrat à imposer ses exigences dans le domaine de la gestion des ressources humaines, ce qui a notamment permis de développer par la suite le concept de développement de l'encadrement.

9. Compétitivité et sécurité de l'emploi : une obligation de contracter a été fixée pour les relations internes. Elle devrait être utilisée pour instaurer les mêmes conditions de concurrence dans un délai d'adaptation. Cela signifie que les prestations de la régie doivent être comparées à celles du marché dans les mêmes conditions, mais que, en cas de coûts plus élevés dans la production publique, cette dernière dispose de mesures de protection pour une durée de cinq ans. Si cette convention a mis tous les pôles annexes à l'abri de la privatisation pendant dix ans, la peur des pôles concernés de perdre éventuellement des marchés (même sans risque) n'a jusqu'à présent pas abouti à une compétition basée sur la comparaison. Dans un seul cas, celui du nettoyage des bâtiments, la ville de Detmold a pris ses distances à l'égard de la privatisation, dans un processus d'adaptation sans précédent, ce marché étant caractérisé par des conditions générales inégales. Dans ce cas précis, le Personalrat a réussi à imposer une « hausse politique des prix » pour l'emploi des femmes dans des conditions correctes.

Avec ces six champs d'action et les neuf mesures citées ci-dessus à titre d'exemples, le Personalrat a tenté de soutenir le processus de réorganisation ou d'imposer par sa persévérance des alternatives répondant à l'objectif, négligé de son point de vue, de priorité à accorder au salarié. Son propos était d'une part d'atténuer les conséquences de la hausse de productivité liée à cet objectif et, d'autre part, de dégager des avantages pour les salariés. Cette tentative s'appuyait sur la conviction que, dans les périodes de transformation, si les changements menacent la sécurité et la visibilité, les gens ont besoin d'être accompagnés et rassurés. Le Personalrat était et reste tout aussi persuadé qu'une réorganisation telle que celle prévue et en cours à Detmold est indispensable pour préserver une fonction publique efficace, et que c'est la seule condition pour garantir les emplois. Il était évident pour le Personalrat que seul un partage des problèmes entre employeur (sécurité de l'emploi et offre de qualification) et salariés (ouverture à la formation et au changement) permettrait, d'une part, de consolider durablement la capacité d'action du service et, d'autre part, d'accroître l'employabilité des personnels.

\section{Assurer la capacité d'action et l'employabilité}

Les capacités d'action et l'employabilité ont été soumises au cours du processus de transformation, du fait de la détérioration des conditions générales, à des exigences de plus en plus grandes. La situation financière continuait de se dégrader, tout comme celle du marché de l'emploi, ce qui renforçait encore chez les salariés la peur du changement. Il fallut également lutter en permanence contre des tentatives d'externalisation (par exemple, nettoyage des bâtiments, ramassage des ordures, espaces verts) ce qui n'était pas pour apaiser la situation. Mais la transparence établie en matière de coûts et de prestations fut utile, car elle permettait de comparer plus objectivement les offres de tiers aux prestations du service public et d'effectuer le processus interne d'adaptation dans les délais prévus.

\section{Compétitivité et externalisation}

Sur la question de l'externalisation de certaines prestations (à l'avenir, quels services la ville doit-elle produire elle-même ?) et de la concurrence avec le privé, le Personalrat a 
de nouveau misé sur son nouveau rôle, consistant non pas à empêcher en s'opposant radicalement, mais à prendre de l'influence en proposant des alternatives. Cette position demandait du courage et de la conviction car nos partenaires traditionnels, les syndicats, comme les salariés, demandaient un refus clair et net. La comparaison leur semblait trop risquée. Or, le Personalrat était convaincu qu'une opposition de principe ne permettrait pas à la municipalité de l'emporter dans la confrontation avec le marché d'autant que le poids de l'idéologie est fort dans ce domaine. Une comparaison (benchmark) avec le privé, sur la base de conditions justes, pouvait selon le Personalrat, après un certain temps d'adaptation éventuel, permettre à la régie de l'emporter. Seules des prestations compétitives garantiraient la sécurité de l'emploi et renforceraient l'acceptation du service public dans l'opinion.

Les processus de production des services dans la fonction publique ne sont toutefois pas toujours déterminés par des orientations rationnelles; ils sont également soumis à des prises d'influence des milieux politiques ou des lobbys. Ce sont les cadres, qui avaient jusque-là piloté les processus dans ces conditions, qui se sont majoritairement montré le plus sceptiques : des procédures transparentes et déhiérarchisées signifiaient pour eux une nouvelle répartition du pouvoir, et requéraient en outre une grande force de persuasion. Cela vaut non seulement pour la direction, mais aussi, de la même manière, pour le Personalrat.

\section{Pouvoir et contrepouvoir dans une nouvelle organisation stratégique}

Le pouvoir et le contrepouvoir ne peuvent plus s'appuyer sur des règles et normes familières ; ils sont sans cesse mis à l'épreuve de la concurrence et doivent chercher les meilleures solutions, une configuration qu'il n'est à notre sens pas pertinent de qualifier de «co-management». La décentralisation des responsabilités et donc l'émancipation des employés font plutôt évoluer les rôles de la direction et du Personalrat vers la "gestion d'intérêts ». Les positions respectives des usagers et des salariés du secteur public n'ont pas fondamentalement changé. Les prestations restent soumises à la pression des coûts et de la justification, et les salariés continuent de rechercher la sécurité, la visibilité et des revenus suffisants. Seules les méthodes et, dans certains cas, les moyens de représentation de ces intérêts contradictoires sont soumis à un processus d'adaptation, caractérisé comme il l'a toujours été par ses acteurs et par le déséquilibre existant le plus souvent. C'est pourquoi, à côté du processus participatif de développement des organisations, le blocage organisé fait aussi partie du répertoire, et l'expérience montre qu'un processus avancé de participation n'y changera vraisemblablement rien.

\section{Synthèse}

L'actuelle loi sur la représentation du personnel est, en matière de droits de participation, insuffisamment adaptée aux processus de modernisation basés sur le développement des organisations. Dans ce domaine, le Personalrat en est réduit à faire des concessions à la direction (générale) des services. En présentant ses conceptions de manière stratégique, le travail de représentation des intérêts du personnel peut encore gagner en efficacité.

La situation financière des municipalités fait peser sur les processus de modernisation d'importantes contraintes budgétaires impliquant notamment de soumettre l'action 
de l'administration à une logique économique et de négliger des objectifs qualitatifs, ce qui rend plus difficile l'acceptation du changement.

Les stratégies de décentralisation des responsabilités et de budgétisation augmentent le risque de désolidarisation de groupes de salariés, tant au niveau collectif qu'individuel. La réussite est mesurée d'abord à l'aune des objectifs de coûts et/ou de recettes.

Les contraintes budgétaires empêchent en outre de faire évoluer les prestations de l'administration. On s'accroche à des prestations dépassées ou inefficaces, ce qui complique la mise en place de nouvelles prestations dont la population a besoin.

La réduction des perspectives aux aspects financiers, aggravé par le manque de moyens, a entraîné un déséquilibre dans la poursuite des objectifs. Dans son rapport avec la performance et l'efficacité, la qualité a souvent été reléguée à l'arrière-plan. L'équilibrage des objectifs à l'aide d'un tableau de bord (Balanced Score Card, BSC), proposé par une société de conseil, en est resté au point mort car il était évident que seules les données financières entraient en considération. L'imagination manquait peutêtre pour prendre en compte la mission de la municipalité. On n'échappe apparemment pas à la logique de faire des économies.

La participation des personnes concernées (salariés, Personalrat, conseil municipal et administrés) dépend très fortement de la position des décideurs. Garantir l'émancipation a été et reste la mission centrale du Personalrat afin d'assurer un équilibre entre les acteurs et de sécuriser largement les processus de transformation. Le caractère décisif de la participation et de la délégation des responsabilités comme facteurs de valorisation dans le processus de transformation est négligé, pour des raisons de conservation du pouvoir (conservatisme structurel).

La clé d'une modernisation réussie réside dans une gestion pertinente des ressources humaines, mais, du point de vue de la représentation du personnel, cette dernière n'est pas toujours reconnue comme telle. Une organisation hiérarchique établie au fil des ans n'est pas toujours synonyme de frustration, c'est aussi une garantie de fiabilité pour les projets de vie des salariés. En l'absence de gestion compétente des RH, le démantèlement d'une organisation familière génère la peur et des réactions de défense. Le Personalrat doit donc avoir une vision à long terme et du courage pour opposer aux incertitudes d'un processus ouvert de modernisation l'outil de qualification.

Comme le montre l'expérience réalisée à Detmold, la représentation du personnel, en l'occurrence le Personalrat peut, en participant, parvenir à stimuler le développement des RH, s'il n'abandonne pas sa position critique et sa partialité en faveur des intérêts des salariés. Mais comme la participation au processus de transformation est insuffisamment garantie par la loi sur la représentation du personnel, il faut un soutien syndical compétent et un engagement politique non partisan. Les syndicats, tout comme les Personalräte, seront ainsi amenés à reconsidérer leurs rôles et leurs stratégies pour les adapter aux nouvelles conditions.

EN RÉSUMÉ, les expériences du Personalrat peuvent se décrire de la manière suivante :

L'augmentation de l'efficience et de l'efficacité véhiculée par les concepts de New Public Management (NPM) ne permettra pas d'échapper aux mesures de consolidation budgétaire, mais cette dernière s'en trouvera déséquilibrée et donc perdra de sa crédibilité auprès de l'opinion et des salariés. 
Le contrôle de gestion introduit par le NPM est jusqu'à présent trop basé sur l'évaluation des coûts et trop peu sur l'évaluation des effets, et dégénère ainsi trop souvent en une « bureaucratie des chiffres », surpassant même celle des textes juridiques.

Les problèmes complexes relatifs aux marchés publics ne sont pas devenus plus faciles à résoudre sous le seul effet des mesures de NPM.

La gestion des RH doit faire face à des défis considérables. Or, c'est la composante la plus négligée du NPM.

Les espoirs que les mesures de NPM rendent plus transparente la concurrence entre État et entreprises privées ont été déçus du fait d'une définition encore insuffisante des exigences et de la qualité.

La représentation du personnel s'est compliquée dans le «triangle » formé par les attentes des salariés, la réalité des conditions générales et la faisabilité de la participation et de l'autonomie dans un contexte de peur de la perte d'emploi.

Le Personalrat est « condamné » au rôle (important) de « gardien » de la gestion des $\mathrm{RH}$ et devient donc un facteur de réussite ou d'échec des prestations publiques.

La délégation croissante des responsabilités et l'émancipation grandissante des salariés requièrent une évolution du rôle du Personalrat : de « co-décideur » à conseiller «protecteur», contrôleur ( gardien ») et «consultant », proposant des alternatives, organisant l'aide à l'autonomie et faisant office de médiateur en cas de conflit, mais étant également en situation d'organiser la résistance si les intérêts des salariés sont négligés. Il n'existe pas d'alternative réelle et efficace au rôle de co-décideur du Personalrat, les arguments et les alternatives ayant toujours su convaincre, comme le montre la réélection à plusieurs reprises du Personalrat. 


\title{
Le dialogue social dans la fonction publique d'Etat en France
}

\author{
Marie-Claude KERVELLA
}

En France, le statut des fonctionnaires d'Etat, adopté en 1946 et complété depuis par diverses législations, détermine les fondements juridiques de la fonction publique. II précise également les modalités de la participation et de la consultation des agents fonctionnaires et de leur représentation, mais sans pour autant donner de cadre juridique à la négociation. Certes, des procédures de négociation se sont progressivement mises en place au cours des trente dernières années, mais hors cadre bien défini et alors même que les lieux et niveaux de concertation semblent aujourd'hui inefficients. A l'heure où la fonction publique est confrontée à des enjeux lourds de conséquences et s'engage dans un mouvement de transformation, une reconfiguration des principes de la concertation peut être jugée souhaitable. Cette position n'est pourtant pas partagée par l'ensemble des partenaires sociaux, ni même au sein de l'Etat employeur. Le diagnostic présenté ici par Marie-Claude Kervella, secrétaire générale de l'Uffa-CFDT,1 est celui de l'organisation syndicale de représentation des fonctionnaires de la confédération CFDT, représentative au niveau nationale, qui prône une révision des règles du dialogue social dans la fonction publique. Cette contribution est centrée sur la fonction publique d'Etat, dont les règles de fonctionnement demeurent déterminantes pour les trois fonctions publiques, même si la vitalité du dialogue social semble différer selon les spécificités propres à chacune.

Une des caractéristiques de la CFDT est d'avoir opté, dans les années 70, non pour une fédération unique de fonctionnaires mais pour une organisation, l'Uffa-CFDT (Union des fédérations de fonctionnaires et assimilés) dans laquelle les fonctionnaires qui y adhèrent sont en premier lieu membres d'une fédération professionnelle. Les fonctionnaires peuvent ainsi être affiliés à une fédération ne regroupant que des fonctionnaires, comme par exemple pour l'enseignement (Fédération de syndicats généraux de l'Education nationale-SGEN) ou au sein de la Fédération CFDT-Finances, ou bien seront représentés au côté des salariés du secteur privé : c'est le cas pour la représentation des personnels hospitaliers (Fédération des services de santé et services sociaux CFDT Santé-Sociaux) ou de ceux du secteur public agricole (Fédération générale agroalimentaire FGA-CFDT). Ce choix, fait pour éviter tout corporatisme, explique bien souvent les positions défendues, puisque toutes auront été discutées bien sûr entre les fonctionnaires, mais aussi avec les salariés du privé. La CFDT essaie ainsi d'avoir une approche la plus interprofessionnelle possible des sujets abordés.

Un paysage syndical complexe

En France, le paysage syndical reste très complexe, notamment dans la fonction publique. Si, au niveau général (secteurs public et privé confondus), on compte cinq

\footnotetext{
${ }^{1}$ CFDT : Confédération française démocratique du Travail
} 
grandes confédérations, on recense au niveau de la fonction publique sept organisations syndicales considérées comme représentatives au niveau national, ce qui signifie qu'elles siègent au Conseil supérieur de la Fonction publique de l'Etat : la CGT, la FSU, l'UNSA, la CFDT, FO, la CGC et la CFTC. ${ }^{2}$ Pour autant, d'autres organisations existent et peuvent être représentatives dans certains secteurs. La tendance observée fait d'ailleurs qu'en cas de dissension au sein d'une organisation, on assiste bien souvent à la création d'une autre organisation syndicale et donc à une nouvelle complexification du paysage syndical. Si on a pu croire, dans les années $80-85$, à une certaine recomposition des organisations syndicales, ce ne fut finalement pas le cas et il faut composer maintenant avec des alliances à géométrie variable, selon les sujets, les périodes, les intérêts en présence. Cette situation initiale vient accroître la difficulté à mettre en œuvre le dialogue social. Mais malgré des positions parfois fort divergentes, du fait de l'histoire spécifique de chaque organisation syndicale, il faut souligner que les organisations syndicales entretiennent des liens entre elles et que les rencontres sont régulières, y compris sur les sujets les plus conflictuels, ceci afin de connaître en amont de toute rencontre avec le ministre la position qui sera défendue par les autres organisations. Et même dans des moments aussi conflictuels que la réforme de 2003 du régime de retraite dans la fonction publique, les relations ont été maintenues avec les autres organisations syndicales à la surprise de beaucoup, y compris quelque fois au sein de la CFDT.

\section{Un dialogue social réduit et fortement institutionnalisé}

C'est donc bien sur fond de paysage syndical complexe que s'organise le dialogue social. Or, il est important de souligner qu'il n'existe en France qu'un dialogue social consultatif. Autrement dit, la négociation n'existe pas. Tous les lieux de discussion sont dits consultatifs, c'est-à-dire que l'avis qui en émerge est simplement recueilli par l'administration. Il arrive ainsi très souvent que des textes soient publiés en dépit d'un vote unanimement négatif des organisations syndicales mais par la seule décision du ministre de les mettre en œuvre. Dès lors, l'opinion répandue selon laquelle les organisations syndicales ne veulent pas négocier est faussée : elles ne le peuvent pas, la négociation n'existant pas puisque l'Etat ne négocie pas. S'il agit là pour la CFDT d'un point sur lequel il importe d'évoluer. D'autres organisations syndicales restent pour leur part très attachées à ce système consultatif, où il n'y a pas de cogestion et où l'Etatemployeur décide de ce qu'il doit mettre en œuvre. On assiste donc là à un dialogue social sous une forme uniquement d'information, puis ensuite de consultation, le gouvernement prenant une décision unilatérale.

Par le passé, on a, il est vrai, déjà parlé d'accords contractuels dans la fonction publique : accords salariaux, accords sur les qualifications, etc. Ces accords, sans force juridique, n'en avaient en fait que le nom, dans la mesure où ils n'engageaient aucun des partenaires, qu'il s'agisse du gouvernement ou des organisations syndicales, à respecter ce qui y était inscrit. Mais il est vrai également qu'en réalité, les accords ont toujours été

\footnotetext{
${ }^{2}$ CGT : Confédération générale du travail

FSU : Fédération syndicale unitaire

UNSA : Union nationale des syndicats autonomes

CFDT : Confédération française démocratique du travail

FO : Force ouvrière

CFE-CGC : Confédération française de l'encadrement CGC

CFTC : Confédération française des travailleurs chrétiens
} 
respectés. L'Uffa a ainsi beaucoup travaillé sur la question du dialogue social dans la fonction publique en France qui a fait par ailleurs l'objet d'un rapport très intéressant (Fournier, 2002) commandé par l'ancien ministre de la Fonction publique Michel Sapin, mais qui, rendu public un mois avant que les élections ne changent la majorité, est resté lettre morte. Et les ministres qui se sont ensuite succédés ont tous souligné l'intérêt des solutions préconisées mais ont finalement rapidement reculé au regard de la difficulté à faire évoluer les formes du dialogue social.

La complexité du dialogue social relève également de la structure des lieux où il se pratique. Il existe pour la fonction publique en France trois Conseils supérieurs : un pour la fonction publique de l'Etat, un pour la fonction publique territoriale, un pour la fonction publique hospitalière. Or dans les faits, le Conseil supérieur de la Fonction publique de l'Etat décide pour tous les autres et passe pour le lieu institutionnel du dialogue social. Pour la CFDT, il s'agit là d'une anomalie qu'il conviendrait de faire évoluer mais qui subsiste pour l'instant. A l'occasion de grandes discussions sur des thématiques-clé comme la question des salaires, les trois fonctions publiques sont certes présentes, mais cette configuration n'est pas institutionnelle : c'est en effet le ministre qui invite les représentants des salariés à venir se réunir au sein d'un groupe de travail informel. Il existe néanmoins deux autres commissions institutionnelles inscrites dans les textes : l'Observatoire de l'Emploi public, instance récente mais extrêmement intéressante qui a notamment produit des chiffres fiables et indiscutables sur la réalité de l'emploi public en France. Cet observatoire, sous la direction de Catherine Zaidman, a également beaucoup avancé sur deux notions encore nouvelles dans l'administration française, et en particulier l'administration de l'Etat, à savoir la gestion des ressources humaines et la gestion prévisionnelle des effectifs, des emplois et des compétences (GPEEC). C'est avec cet observatoire que nous avons pu travailler à la réalisation de guides méthodologiques pour les administrations leur permettant d'élaborer leur plan GPEEC. Il ne s'agit toutefois pas là d'une instance paritaire, puisqu'elle est composée essentiellement de représentants des élus. Enfin, il existe une dernière instance qui, en toute logique, devrait être précisément celle où l'on discute des réformes en cours et à venir : la Commission de modernisation. Elle-même assez récente puisqu'elle date des années 2000, cette instance composée de représentants des élus, de l'administration et des organisations syndicales, mais également de représentants des usagers, se réunit malheureusement extrêmement peu souvent. C'est pourtant à elle qu'aurait dû revenir l'accompagnement des réformes récentes, notamment dans le cadre de la LOLF (nouvelle Loi organique relative aux lois de finances) et de la réorganisation de l'Etat au niveau territorial. Or, voilà maintenant un an et demi qu'elle ne s'est pas réunie en formation plénière, en dépit des demandes réitérées auprès des ministres successifs de la Fonction publique qui semblent préférer réunir des instances plus faciles à manager.

\section{Un dialogue social en panne}

On est donc en présence, d'une part, d'une forme de dialogue social dont les règles du jeu sont complexes et n'incitent pas à la négociation et, d'autre part, de lieux de discussion soit très institutionnalisés, comme les conseils supérieurs, soit très peu réunis, car ils sont nouveaux et s'avèrent plus compliqués à gérer pour les ministres. On peut donc être là très critique par rapport à la réalité du dialogue social et donc à la place que les organisations syndicales prennent dans un processus de réforme. Nous disons couramment que nous vivons une panne historique de dialogue social. Les accords qui 
ont pu être signés dans les années 90 sont maintenant absents depuis pratiquement dix ans : absence d'accords sur les salaires, absence d'accords sur la formation, etc. Les partenaires sociaux n'ont pas non plus réussi à obtenir un accord sur la réduction du temps de travail qui, pour la CFDT, semblait pourtant indispensable puisqu'il aurait permis de travailler sur l'ouverture des services publics, les conditions de travail, l'emploi et éventuellement la réorganisation de l'emploi dans les administrations sur l'ensemble du territoire. Cette question a fait l'objet d'un décret uniquement. Or, l'absence d'accordcadre sur la réduction du temps de travail n'est pas tant le fait des organisations syndicales, hostiles ou non, mais de l'administration et des ministères qui n'auront pu s'entendre entre eux sur l'intérêt de conclure un accord-cadre sur ce sujet. En France, chaque ministre demeure en effet très jaloux de ses prérogatives et souhaite pouvoir, dans son ministère, manager comme il le souhaite ses équipes, dans le respect bien sûr du statut. On constate ainsi que, sur la question de l'organisation du travail, chaque ministre préfère rester maître chez lui, d'autant plus si les moyens financiers qui lui sont octroyés sont conséquents.

On pourrait ainsi citer l'exemple du ministère des Finances, farouche partisan du non-accord, mais quelques fois aussi d'autres ministères comme celui de l'Intérieur. De manière générale, les petits ministères se révèlent plus favorables à des accords transversaux leur permettant d'avoir un cadrage pour les réformes qu'ils ont à mener, tandis que les ministères plus importants restent très indépendants. On pourrait ainsi évoquer la question des rémunérations qui demeure l'un des points les plus inégalitaires dans la fonction publique en France puisque, sous couvert d'une grille de salaires qui est la même pour tous, la réalité des rémunérations révèle d'extrêmes différences dès lors qu'on prend en compte les rémunérations annexes. Le mérite n'a alors rien à voir, c'est simplement l'appartenance à tel corps et/ou telle administration qui en est la cause. Or, la question de la rémunération constitue bien là un énorme champ revendicatif puisque globalement, selon le ministère d'appartenance, le montant des primes peut varier entre l'équivalent d'un mois de rémunération et le doublement du salaire sur l'année pour un même poste (à travail, volume horaire et note équivalents).

La réalité du dialogue social peut donc être apparentée à une panne historique dans un moment-clé où sont mises en œuvre la LOLF et la réforme de l'administration au niveau territorial. Or, dans ce contexte, aucun véritable débat national n'a été mené sur le type de service public voulu, sur la nature des missions de l'Etat et sur la façon dont on parviendra à articuler le service public entre le niveau de l'Etat et celui des administrations territoriales. Pour la CFDT, ce débat national doit être mené. Or, les gouvernements successifs s'emploient toujours à mettre la charrue avant les bœufs : faire des économies impliquant de supprimer des emplois puis seulement en conséquence, s'interroger sur les missions à conserver ou à abandonner. De l'avis de l'Uffa-CFDT, la réalisation d'économies et la suppression éventuelle d'emplois doivent être précédées d'un débat de portée nationale où le parlement, mais également l'ensemble des citoyens pourront se prononcer sur la définition des périmètres de l'Etat et de ses missions, afin de tirer ensuite les conséquences en termes d'emplois. Nous sommes donc confrontés à une réalité difficile pour le dialogue social dans ces processus de réforme qui, en retour, interdit toute appropriation des réformes par les fonctionnaires eux-mêmes. Les agents sont ainsi tenus très loin du débat sur le contenu des réformes et leur mise en œuvre alors même que les réformes ne peuvent se faire ni sans ni contre eux : elles ne peuvent se faire qu'avec eux. Or, tant qu'on continuera à 
décider de réformes à l'extérieur de la fonction publique et à vouloir les mettre en œuvre de façon quelque peu occulte, on ne fera pas adhérer les agents aux processus de modernisation.

\section{Moderniser pour promouvoir un service public de meilleure qualité}

Dans ce contexte, quels sont, pour la CFDT, les points que nous souhaiterions voir avancer, à la fois dans l'évolution du dialogue social et ces processus de réforme ? Nous pensons tout d'abord qu'un point fort qu'il faut affirmer est la place du service public au cœur des réformes : d'une part, les réformes doivent avant tout avoir pour objet d'améliorer le service public; d'autre part, en termes de service public, les trois fonctions publiques doivent être à égalité de droits et de devoirs et il doit exister une réelle complémentarité entre les trois versions de la fonction publique. Peu importe qu'un service public soit mis en œuvre par la fonction publique territoriale ou par l'Etat, si celui-ci est bien effectué et, à notre avis, à la fois au plus près des agents et des usagers, ce qui constitue un facteur de contrôle démocratique de la bonne mise en œuvre du service public.

\section{Rénover les statuts et les structures d'emploi}

Le deuxième point est que nous pensons qu'il faut aller vers une évolution du statut de la fonction publique qui se traduira notamment par une réforme importante du nombre de corps. Bien avant que le ministre Renaud Dutreil et le rapport Pochard (Conseil d'Etat, 2003) n'évoquent cette question qui, il faut le préciser, n'a jamais été mise en débat ni en discussion officiellement, cette revendication figurait déjà dans la plateforme revendicative de l'Uffa-CFDT. Nous pensons en effet que l'actuel découpage en 900 corps de la seule fonction publique d'Etat s'avère avant tout un système extrêmement bloquant pour les déroulements de carrière des agents. Qu'il s'agisse de mobilité ou de promotion, ce système de corps qui érige des protections pour préserver les postes d'avancement se révèle, à notre sens, extrêmement désavantageux pour les carrières des agents. Il est vrai qu'une réforme aussi importante que celle aujourd'hui évoquée, où l'on passerait de 900 corps à 28 ou 30 cadres d'emplois, demande à être énormément travaillée. Toute la difficulté réside en effet dans la capacité d'expliquer aux agents qu'ils conserveront un statut garanti, garderont les protections actuelles mais devront en contrepartie accepter de nouvelles règles et adopter de nouveaux modes de gestion. Cette rénovation des statuts nous semble néanmoins indispensable car nous estimons que, dans le cas contraire, nous irons vers une fonction publique de plus en plus réduite ne conservant, sous forme de gestion par corps, que les missions régaliennes de l'Etat. Les autres missions seraient très rapidement externalisées, la gestion des corps n'offrant alors plus les moyens d'effectuer réellement les missions de service public. Il s'agit donc pour la CFDT d'une réforme incontournable, mais qui, de l'avis des responsables politiques, ne peut être mise en œuvre à n'importe quel moment. Autrement dit, un gouvernement ne prendra jamais la responsabilité de se lancer dans une telle réforme avant des échéances électorales importantes.

Par ailleurs, cette éventuelle simplification des corps se heurte à une alliance objective entre un certain nombre d'organisations syndicales et de ministères qui voient ces projets de réforme d'un très mauvais œil puisqu'ils entraîneront pour eux la perte de certaines citadelles. Autrement dit, le ministère des Finances ou le ministère de l'In- 
térieur aimeraient garder les structures en emploi telles qu'elles sont actuellement. D'autres organisations, dont $\mathrm{FO}$, la plus hostile à cette réforme, estiment que ce sera un moyen d'accélérer un mouvement de suppression des effectifs et d'aller vers une gestion plus individualisée des agents qu'elle ne l'est aujourd'hui. Il faut savoir aussi que l'organisation interne de FO est fondée sur des syndicats de corps et que la suppression de ces derniers détruirait tout l'équilibre politique interne de cette organisation. Ce qui amène l'organisation syndicale à déclarer ouvertement au ministre qu'elle ne cautionnera pas une réforme qui l'obligerait à transformer entièrement son organisation interne.

\section{La rénovation du dialogue social au cœur de la modernisation}

Enfin, troisième point sur les évolutions souhaitées par la CFDT pour que les organisations syndicales puissent être de véritables partenaires dans la modernisation du service public: la rénovation en profondeur du dialogue social dans la fonction publique. La CFDT se prononce ainsi pour l'introduction de la notion de politique contractuelle dans la fonction publique, avec la signature d'accords dotés d'une véritable valeur juridique. Par ailleurs, un certain nombre de sujets, comme les salaires, devraient être régulièrement soumis à la négociation. Ce n'est actuellement pas le cas car il n'y a rencontre salariale que si le ministre le veut et qu'il dispose de marges budgétaires suffisantes. Dans le cas contraire, il y a une décision unilatérale d'augmentation, sans que les partenaires sociaux se soient seulement assis autour d'une table. Nous pensons donc que les salaires, mais aussi les conditions de travail, la formation professionnelle, les perspectives de promotion, en clair tout ce qui est du ressort du statutaire et du déroulement de carrière, devraient faire partie de négociations régulières obligatoires. Nous estimons également que, si on parvenait à de véritables négociations débouchant sur des accords formels, il faudrait en conséquence mettre en œuvre la notion d'accord majoritaire, moyen efficace de responsabilisation de l'ensemble des partenaires. Car lorsqu'on arrive à un accord à l'issue d'un processus de négociation, il faut pouvoir justifier le cas échéant des raisons pour lesquelles on ne le signe pas. Les organisations syndicales ont beaucoup avancé sur cette possibilité avec des ministres précédents, sans pouvoir aboutir, mais cette responsabilisation des organisations syndicales serait un moyen extrêmement important de mieux participer à la modernisation de l'Etat.

Un autre point qui, à notre avis, serait tout à fait indispensable, c'est de mettre en place de nouveaux lieux de dialogue social. En effet, le processus de réorganisation de l'Etat accroît considérablement l'importance du préfet de région, entouré des chefs de services régionaux ( 8 pôles et 8 chefs de service autour de lui) : or il n'existe à cet échelon aucun lieu de dialogue social alors que des décisions y sont prises. Il faudrait donc instaurer des lieux de dialogue social interministériels et régionaux. Dans le cadre de la LOLF également, on pourrait imaginer qu'autour des chefs des budgets opérationnels de programme se mettent en place des lieux de concertation, à géométrie variable et temporaires, au fur et à mesure que les BOP (budgets opérationnels de programme) pourraient évoluer : des décisions vont en effet être prises à cet échelon, y compris en termes de gestion du personnel, mais il n'existe aujourd'hui aucun lieu de concertation à ces niveaux précis.

Enfin, nous pensons qu'il est assez désolant, alors qu'on discute de la LOLF depuis 5 ans, que les conséquences en matière de gestion des ressources humaines aient été si peu discutées. On sait que la réforme aura un impact, notamment sur la question des promotions et des mobilités, mais sans avoir anticipé ni formalisé ces aspects précis. 
Une nouvelle gestion des personnels sera donc mise en œuvre dès 2006, mais à tâtons. Cette situation va, après tout, peut-être pousser à des expérimentations susceptibles d'aboutir à des évolutions intéressantes, mais à l'heure actuelle, les perspectives demeurent floues en matière de LOLF et de gestion des ressources humaines. Nous préconisons donc une formalisation rapide et la mise en place auprès des gestionnaires locaux de responsables de la gestion des ressources humaines, individus ou centres ressources, capables d'aider les responsables, qui sont souvent des responsables techniques, à prendre en charge localement la gestion des personnels, de façon à ce que la conséquence des réformes ne soit pas néfaste mais soit bien au contraire un levier pour un meilleur déroulement de carrière dans l'administration.

\section{Indications bibliographiques}

Conseil d'Etat, Rapport public 2003 : jurisprudence et avis 2002. Perspectives pour la fonction publique, Paris, La documentation française, 2003, 446 pages (Etudes et documents du Conseil d'Etat $\mathrm{n}^{\circ}$ 54)

FOURNIER J., Livre blanc sur le dialogue social dans la fonction publique, Paris : rapport au ministre de la Fonction publique et de la réforme de l'Etat, La Documentation française, 2002, 208 pages 



\section{Les auteurs}

\section{Pierre BAUBY}

Président de la Commission des services d'intérêt général du CEEP (Centre européen des entreprises à participation publique et des entreprises d'intérêt économique général), Bruxelles

\section{Rina BOHLE ZELLER}

Consultant senior chez Pricewaterhouse Coopers, Paris

\section{Dietmar FRINGS}

Directeur de l'association patronale Telekom

Responsable des Relations internationales, Deutsche Telekom AG, Bonn

\section{Hermann HIBBELER}

Ancien président du Personalrat de la ville de Detmold

\section{Marie-Claude KERVELLA}

Secrétaire générale de l'Uffa-CFDT

(Union des fédérations de fonctionnaires et assimilés), Paris

\section{LeO KIBLER}

Professeur de sociologie à l'université Philipps,

Institut de Sociologie, Marburg

\section{Susanne KÖNIG}

Chargée de recherches auprès de l'Institut de gestion et de pédagogie économique, Université Carl von Ossietzky, Oldenburg

\section{Sabine KUHLMANN}

Chargée de recherches auprès de la Faculté des Sciences économiques et sociales, Université de Potsdam

\section{René LASSERRE}

Professeur à l'Université de Cergy-Pontoise, Directeur du CIRAC, Cergy-Pontoise

\section{Philippe MONCOURRIER}

Direction des Ressources Humaines, RATP, Paris 
Marie-Hélène PAUTRAT

Ingénieur d'études au CIRAC, Cergy-Pontoise

\section{Karsten SCHNEIDER}

Chercheur à la Fondation Hans-Böckler, Düsseldorf

\section{Catherine ZAÏDMAN}

Secrétaire générale de l'Observatoire de l'emploi public, Paris 
Composition : CIRAC

CIRAC, c/o Université de Cergy-Pontoise

33 Boulevard du Port - 95011 CERGY-PONTOISE CEDEX

Impression : Dupli-Print

2, rue Descartes

ZI SEZAC - 95330 DOMONT

Dépôt légal : juin 2007

Imprimé en France 



\section{Modernisation des services publics et management social en France et en Allemagne}

Sous la direction de Leo KIßLER, René LASSERRE et Marie-Hélène PAUTRAT

En Allemagne comme en France, le service public est confronté au défi de la modernisation sous la pression de multiples facteurs : consolidation des budgets publics, mise en concurrence et dérégulation des services d'intérêt général à l'échelle européenne, évolution des attentes des usagers-clients au regard de la flexibilité et de la qualité des services rendus, nécessité de promouvoir à l'échelon des Etats et des régions un environnement public stimulant pour l'activité économique...

Ces changements imposent une rénovation en profondeur des modèles classiques d'organisation de l'action publique et, en tout premier lieu, la mise en œuvre de nouvelles formes de management des ressources humaines. Le présent ouvrage rassemble diverses contributions de chercheurs et responsables français et allemands portant sur la gestion de l'emploi et des compétences, la représentation du personnel et les relations sociales dans la sphère publique. Il en ressort un tableau différencié qui met en évidence les conditions et les modalités très variables selon lesquelles ces grands enjeux de la modernisation de l'Etat sont abordés de part et d'autre du Rhin.

$20 € \mathrm{TTC}$

ISBN : 978-2-905518-34-7

\section{CIRAC}

